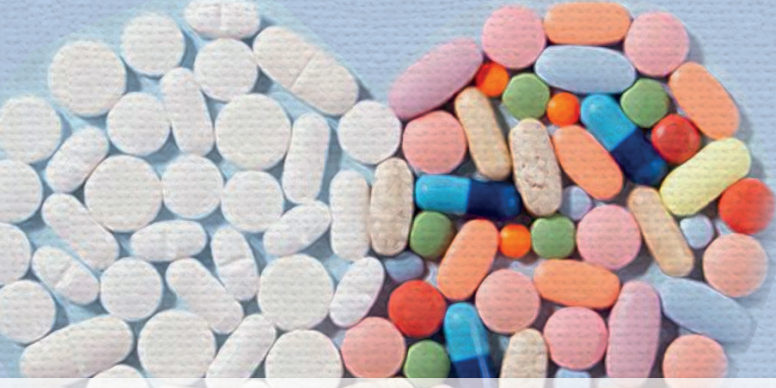

Safety and efficacy of

\title{
cardiovascular medication
}

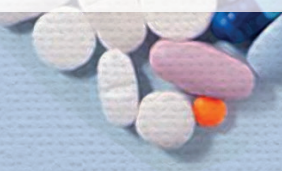

in women

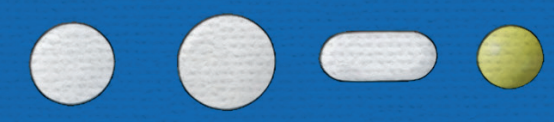

\section{and men}

$\infty$

$\infty 0$

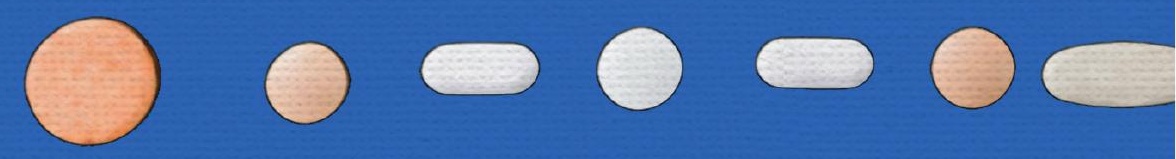

- the added value of

- clinical care data

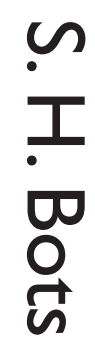



Safety and efficacy of cardiovascular medication in women and men; the added value of clinical care data

Sophie Heleen Bots 
Safety and efficacy of cardiovascular medication in women and men; the added value of clinical care data

$\mathrm{PhD}$ thesis with a summary in Dutch

Author: Sophie Heleen Bots

Rights:

Please use the content of this $\mathrm{PhD}$ thesis as much as possible, with appropriate references

Lay out and design: $\quad$ Sophie Bots, Iliana Boshoven-Gkini | www.AgileColor.com

Cover illustration: $\quad$ Elies Eijdems, Sophie Bots, Iliana Boshoven-Gkini

Printing: GVO drukkers \& vormgevers B.V., www.gvo.nl

ISBN: 978-94-6332-769-5

Financial support by the Dutch Heart foundation for the publication of this thesis is gratefully acknowledged.

Financial support by Cardiologie Centra Nederland for the publication of this thesis is gratefully acknolwedged. 
Safety and efficacy of cardiovascular medication in women and men; the added value of clinical care data

Veiligheid en werkzaamheid van cardiovasculaire geneesmiddelen in vrouwen en mannen; de toegevoegde waarde van reguliere zorgdata (met een samenvatting in het Nederlands)

\section{Proefschrift}

ter verkrijging van de graad van doctor aan de

Universiteit Utrecht

op gezag van de

rector magnificus, prof. dr. H.R.B.M. Kummeling,

ingevolge het besluit van het college voor promoties

in het openbaar te verdedigen op

donderdag 2 september 2021 des middags te 12.15 uur

door

Sophie Heleen Bots

geboren op 26 februari 1994

te Rotterdam 


\section{Promotor}

Prof. dr. ir. H.M. den Ruijter

\section{Copromotor:}

dr. N.C. Onland-Moret 


\section{Table of contents}

$\begin{array}{lll}\text { Chapter I General introduction } & 7\end{array}$

Chapter 2 Recommended heart failure medications and adverse drug reactions I5 in women: a call for sex-specific data reporting

Chapter 3 Adverse drug reactions to guideline-recommended heart failure drugs in women: a systematic review of the literature

Chapter 4 Routine clinical care data from thirteen cardiac outpatient clinics design of the Cardiology Centers of the Netherlands (CCN) database

Chapter 5 Sex differences in the number and type of adverse drug reactions reported for common cardiovascular medications in a cardiac outpatient population

Chapter 6 Heart failure medication dosage and survival in women and men seen at outpatient clinics

Chapter 7 Statins reduce mortality risk to a greater extent in women compared with men in a cardiac outpatient population

Chapter 8 General discussion

Appendix

Nederlandse samenvatting

Dankwoord

List of publications

Curriculum Vitae 

General introduction 


\section{General introduction}

Women and men are equal but different. They are different from a biological perspective, where we can separate females from males down to the cellular level due to the absence (or presence) of the Y-chromosome. They are also different from a societal perspective, where we label someone as woman or man by comparing their behaviour or physical appearance against existing gender norms. It is important to understand that sex and gender are not interchangeable terms, but rather describe two separate concepts. Sex encompasses biological differences between female and male organisms, such as the presence and effects of sex hormones and sex chromosomes, and the resulting differences in anatomy and physiology. Gender describes social differences between women and men that are engrained in society, such as their expected behaviour in certain situations and the way they are expected to dress, which then shape rules of conduct within society. Both sex and gender play an important role in health and disease, albeit through different mechanisms. Biological differences may predispose either sex to certain conditions' whereas societal differences may lead to differential quality of care due to both gendered interaction between individuals and the healthcare system ${ }^{2}$ and the fact that research underlying evidence-based medicine also incorporates these gendered approaches ${ }^{3}$.

\section{Sex differences in cardiovascular disease and heart failure in specific}

Sex and gender differences in disease have been gaining attention over the last few decades, especially with regard to cardiovascular disease (CVD). Historically considered a man's disease, the two main components of CVD (ischaemic heart disease and stroke) actually affect women and men similarly. Globally, they are the leading causes of death in both sexes ${ }^{4}$, and in those aged 85 years and older women outnumber men in cardiovascular mortality by approximately one million cases ${ }^{5}$. However, women suffering from for example coronary heart disease are less likely to be correctly diagnosed ${ }^{6}$, and remain underrepresented in clinical trials looking into treatments for this condition ${ }^{7}$. This results in a lack of knowledge about how to best diagnose and treat CVD in women, for both CVD subtypes that are common in both sexes and CVD subtypes that occur more frequently in women such as Tako Tsubo ('broken heart') cardiomyopathy and spontaneous coronary artery dissection 8.9 .

The lack of sex-specific knowledge extends to the field of heart failure (HF), a cardiovascular syndrome that currently affects more than 37 million individuals and mainly occurs in the elderly ${ }^{10}$. In the next 50 years, the prevalence of HF is expected to grow to 100 million individuals in the United States alone due to ageing of the population" ", making it a pressing healthcare concern. The syndrome of HF can be subdivided into two main subtypes based on the ejection fraction (EF), depending on whether the contraction or the relaxation of the heart muscle is impaired ${ }^{\prime 2}$. Interestingly, the prevalence of these two subtypes is strongly sex-dependent ${ }^{\prime \prime}$. Women more often present with lower filling capacity due to impaired 
relaxation, or HF with preserved $\mathrm{EF}$ (HFpEF; EF $\geq 50 \%$ ). Men, on the other hand, more often present with lower cardiac output due to impaired contraction, or HF with reduced EF (HFrEF; EF <40\%) ${ }^{12}$. Next to the physiological presentation, the underlying aetiology is also thought to differ between these subtypes. According to the most recent paradigm, HFrEF is driven by direct damage to the cardiomyocytes (heart muscle cells) due to for example a myocardial infarction, whereas HFpEF is driven by dysfunction of endothelial cell (cells lining the blood vessels) that occurs due to a systemic inflammatory state induced by comorbidities such as obesity, hypertension and diabetes mellitus ${ }^{13}$. Treatment strategies also differ between the two HF subtypes, mainly because medication that improves the prognosis of patients with HFpEF is lacking and thus treatment of this syndrome focuses solely on reducing the disease burden ${ }^{12}$. Within each HF subtype, however, current guidelines recommend the same treatment for women and men ${ }^{12}$ despite known sex differences in drug metabolism ${ }^{14}$ that relate to a 1.5-I.7 times higher risk of adverse drug reactions in women receiving cardiovascular medications ${ }^{15}$. Proving whether this 'one size fits all' approach to HF medication is appropriate should be a simple matter, as this approach is based on landmark clinical trials in the field and thus sex-stratified data from these trials should be sufficient to answer the question. However, landmark trials might not be the most suitable place to look for data on women.

\section{Underrepresentation of women in clinical trials and the implications}

Women have historically been underrepresented in clinical trials. While this trend may have originated from a desire to protect women (and their unborn babies) from harm after the thalidomide disaster, it now paradoxically exposes women to potentially dangerous adverse drug reactions ${ }^{16}$. Cardiovascular trials are no exception, and despite continued efforts to correct this imbalance, women remain underrepresented especially in ischaemic heart disease and HF trials ${ }^{17,18}$. In the case of HF, the sex disparity is also maintained by the use of in- and exclusion criteria that favour the male pattern of disease ${ }^{18}$. Typical characteristics of HF in women, such as older age, preserved EF, and several comorbidities, often serve as the exclusion criteria for HF trials, disproportionally excluding women from participation in these trials ${ }^{11,18,19}$. As a result, women comprise only $20-30 \%$ of HF clinical trial populations while they represent $50 \%$ of all HF patients in the general population ${ }^{7}$.

This underrepresentation of women in HF trials directly translates to a lack of womenspecific clinical trial data, posing two important questions related to medication dosage. First, whether currently recommended doses have the optimal treatment effect in women, and second whether currently recommended doses might unnecessarily increase the risk of adverse drug reactions (ADRs) in women. At the moment, the efficacy and safety of new medications are tested in early phase trials that typically include only small groups of either healthy individuals or those with the disease of interest. The underrepresentation of women is even more pronounced in these early phase trials ${ }^{20}$, which means that safe and effective 
medication doses are determined based on data coming mainly from men. This not only complicates any attempts at evaluating sex-specific optimal doses, but also strongly reduces the chances of picking up severe but rare ADRs that affect mainly or only women early in the process.

The assumption underlying this practice is that findings based on men can be safely generalised to women. However, this is called into question by data showing sex differences in body composition, hormones and metabolism that affect both how drugs are absorbed and metabolised by the body (pharmacokinetics) and how the active compounds of those drugs interact with the body (pharmacodynamics) ${ }^{14}$. This implies that, depending on the pharmacological properties of the drug, the concentration in the blood or the period of time it stays in the body may be different for women and men even when they receive the same dose. Beta-blockers (antihypertensive drugs) are a good example as they are cleared faster by the male body compared with the female body, meaning that women are exposed to this drug for a longer period of time when given the same dose as men ${ }^{14}$. Such findings have sparked debate about whether prescribing women and men the same doses is actually appropriate, and the results from a recent study looking into sex-specific optimal doses of HF medication indeed suggested women already reached the optimal treatment effect at half the dose required for men $^{21}$.

Sex differences in drug metabolism may also warrant sex-specific doses for the sake of safety. For example, eight out of the ten drugs withdrawn from the market by the United States Food and Drug Association due to health concerns between 1997 and 2000 posed a greater health risk to women ${ }^{16}$. Literature also suggests that women have approximately twice the risk of adverse drug reactions (ADRs) compared with men ${ }^{15}$. These can be a serious threat to health on the short term and may require hospitalisation ${ }^{22}$, but milder ADRs also compromise health on the long term by reducing adherence to medication ${ }^{23}$ and thus denying women the potential benefits of treatment. To cover for the lack of sex-specific data, researchers have come up with clever observational study designs to at least generate some insights in this matter. For example, by comparing the efficacy of two different medication types used to treat high blood pressure in women and men, they showed that one (angiotensin II receptor blockers; ARBs) was more effective in women than the other (angiotensin-converting enzyme inhibitors; ACEls). Interestingly, they did not see such a difference in men $^{24}$. When you then connect this bit of knowledge to data showing that women treated with ACEls more often experience dry cough symptoms than men ${ }^{25-27}$, it could suggest that ACEl-induced cough reduces adherence in women and that ARBs might be therefore the preferred choice of treatment. However, these kind of thought experiments rely heavily on speculation and are not sufficient to change clinical practice. 
Properly addressing these persisting knowledge gaps is a first step towards defining optimal strategies for women and men with HF that both ensure the best treatment efficacy and reduce the risk of unnecessary ADRs. To achieve this, large amounts of sex-stratified data are needed, but this immediate need for data cannot be solved by clinical trials alone as these take years to complete. In addition, pharmaceutical companies are unlikely to sponsor large-scale trials for medications that have already been proven to work and have lost their patent because this is not cost-effective. This is where the added value of clinical care data comes in.

\section{The added value of clinical care data for sex research in heart failure}

With the advent of stronger computers and digitalised health care systems, came the opportunity to store regular care data and subsequently use these data for research purposes. Clinical care data are unique in that they simultaneously cover a large number of individuals and a large selection of clinically relevant measures, often even across relatively long time periods. In addition, these data are collected as part of daily clinical care and thus are a direct reflection of the patients normally seen by physicians. This makes such datasets highly valuable for research in populations traditionally underrepresented in data collected for research purposes, such as women. These datasets can address many of the existing evidence gaps that exist solely because data have never been available to address them. Changing current research practice into a more sex-considerate environment is important, but as has become clear over the past few decades, progress here is slow. We hypothesize that clinical care data can make valuable contributions to the sex differences research field by generating evidence where there currently is none. As such, these data can breathe new life into the discussion about whether tailoring clinical care to patient sex improves the quality of care for both women and men with HF.

\section{The outline of this thesis}

We show that there is a lack of sex-specific data on adverse drug reactions to guidelinerecommended heart failure drugs in both clinical trial data (Chapter 2 ) and the literature as a whole (Chapter 3). We then introduce a large clinical care database (Chapter 4) and use these data to explore sex differences in the number and type of adverse drug reactions to commonly prescribed cardiovascular drugs (Chapter 5). We also evaluate whether the optimal dosage of guideline-recommended heart failure medication is different for women and men (Chapter 6). We then continue to show that this clinical care database can also be used to address other important cardiovascular medication-related topics in sex differences research (Chapter 7). The last chapter (Chapter 8) puts the findings of this thesis in a broader perspective and discusses the potential of clinical care data in advancing the field of sex differences research. 


\section{References}

I. Klein SL, Flanagan KL. Sex differences in immune responses. Nature Reviews Immunology 20I6; I6(I0): 62638.

2. Heise L, Greene ME, Opper N, et al. Gender inequality and restrictive gender norms: framing the challenges to health. The Lancet 2019; 393(I0I89): 2440-54.

3. Gupta GR, Oomman N, Grown C, et al. Gender equality and gender norms: framing the opportunities for health. The Lancet 2019; 393(10190): 2550-62.

4. Naghavi M, Abajobir AA, Abbafati C, et al. Global, regional, and national age-sex specific mortality for 264 causes of death, 1980-2013;2016: a systematic analysis for the Global Burden of Disease Study 2016. The Lancet 2017; 390(10100): II5I-210.

5. Roth GA, Abate D, Abate $\mathrm{KH}$, et al. Global, regional, and national age-sex-specific mortality for 282 causes of death in 195 countries and territories, 1980-2013;2017:asystematic analysis for the Global Burden of Disease Study 2017. The Lancet 2018; 392(10159): 1736-88.

6. Mehta LS, Beckie TM, DeVon HA, et al.Acute Myocardial Infarction in Women. Circulation 2016; I33(9): 916 47.

7. Scott PE, Unger EF, Jenkins MR, et al. Participation of Women in Clinical Trials Supporting FDA Approval of Cardiovascular Drugs. Journal of the American College of Cardiology 2018; 71 (18): 1960-9.

8. Hayes SN, Kim ESH, Saw J, et al. Spontaneous Coronary Artery Dissection: Current State of the Science: A Scientific Statement From the American Heart Association. Circulation 2018; 137(19): e523-e57.

9. Kato K, Lyon AR, Ghadri J-R, Templin C. Takotsubo syndrome: aetiology, presentation and treatment. Heart 2017; I03(I8): I46I-9.

10. Ziaeian B, Fonarow GC. Epidemiology and aetiology of heart failure. Nat Rev Cardiol 2016; I3(6): 368-78.

II. Dunlay SM, Roger VL, Redfield MM. Epidemiology of heart failure with preserved ejection fraction. Nature Reviews Cardiology 2017; 14(10): 591-602.

12. Ponikowski P,Voors AA, Anker SD, et al. 2016 ESC Guidelines for the diagnosis and treatment of acute and chronic heart failure: The Task Force for the diagnosis and treatment of acute and chronic heart failure of the European Society of Cardiology (ESC)Developed with the special contribution of the Heart Failure Association (HFA) of the ESC. European Heart Journal 2016; 37(27): 2129-200.

13. Paulus WJ, Tschöpe C.A Novel Paradigm for Heart Failure With Preserved Ejection Fraction: Comorbidities Drive Myocardial Dysfunction and Remodeling Through Coronary Microvascular Endothelial Inflammation. Journal of the American College of Cardiology 2013; 62(4): 263-7I.

14. Soldin OP, Mattison DR. Sex differences in pharmacokinetics and pharmacodynamics. Clin Pharmacokinet 2009; 48(3): I43-57.

15. Rosano GM, Lewis B, Agewall S, et al. Gender differences in the effect of cardiovascular drugs: a position document of the Working Group on Pharmacology and Drug Therapy of the ESC. Eur Heart J 20I5; 36(40): 2677-80.

16. Carey JL, Nader N, Chai PR, Carreiro S, Griswold MK, Boyle KL. Drugs and Medical Devices:Adverse Events and the Impact on Women's Health. Clin Ther 2017; 39(I): I0-22.

17. Melloni C, Berger JS, Wang TY, et al. Representation of women in randomized clinical trials of cardiovascular disease prevention. Circ Cardiovasc Qual Outcomes 2010; 3(2): I35-42.

18. Pilote L, Raparelli V. Participation of Women in Clinical Trials: Not Yet Time to Rest on Our Laurels. Journal of the American College of Cardiology 2018; 7 I(I8): 1970-2.

19. Sharpe N. Clinical Trials and the RealWorld: Selection Bias and Generalisability of Trial Results. Cardiovascular Drugs and Therapy 2002; 16(I): 75-7.

20. Pinnow E, Sharma P, Parekh A, Gevorkian N, Uhl K. Increasing Participation of Women in Early Phase Clinical Trials Approved by the FDA.Women's Health Issues 2009; 19(2): 89-93.

2I. Santema BT, Ouwerkerk W, Tromp J, et al. Identifying optimal doses of heart failure medications in men compared with women: a prospective, observational, cohort study. Lancet 201 9; 394(I0205): I254-63. 
22. Rodenburg EM, Stricker BH,Visser LE. Sex-related differences in hospital admissions attributed to adverse drug reactions in the Netherlands. Br J Clin Pharmacol 20I I; 7 I (I): 95-I04.

23. Leporini C, De Sarro G, Russo E. Adherence to therapy and adverse drug reactions: is there a link? Expert Opin Drug Saf 2014; 13 Suppl I: S4 I-55.

24. Hudson M, Rahme E, Behlouli H, Sheppard R, Pilote L. Sex differences in the effectiveness of angiotensin receptor blockers and angiotensin converting enzyme inhibitors in patients with congestive heart failure--a population study. Eur J Heart Fail 2007; 9(6-7): 602-9.

25. Gibson GR. Enalapril-induced cough. Arch Intern Med 1989; 149(I2): 2701-3.

26. Yesil S, Yesil M, Bayata S, Postaci N.ACE inhibitors and cough. Angiology 1994; 45(9): 805-8.

27. Coulter DM, Edwards IR. Cough associated with captopril and enalapril. Br Med J (Clin Res Ed) 1987; 294(6586): I52 |-3. 



\section{Recommended heart failure medications and adverse drug reactions in women: a call for sex- specific data reporting}

2

Sophie H. Bots

Hester M. den Ruijter 


\section{Introduction}

Women treated with guideline recommended cardiovascular medications experience more adverse drug reactions (ADRs) than men'. These women are not only at higher risk of hospitalisation but may also discontinue their medications due to some of the adverse reactions, losing the potential benefit ${ }^{2}$. The safety of cardiovascular medications is evaluated both in clinical trials and through post-marketing surveillance. The latter is important because many ADRs rarely occur in clinical trial populations, which are generally younger and healthier than the target population. This is especially true for women, since their systematic underrepresentation in cardiovascular trials hinders the identification of gender differences in the efficacy and safety of cardiovascular medications. The underrepresentation of women is clearly illustrated in heart failure (HF) trials. While approximately half of all HF patients are women and about $60 \%$ of these women die from this syndrome, on average only $30 \%$ of $\mathrm{HF}$ trial populations are women ${ }^{3}$, possibly due to their older age at HF onset. The evaluation of medication safety in women is further hampered by poor inclusion of sex-specific data in trial reports ${ }^{3}$. The increasing prevalence of the women-dominated HF subtype with preserved ejection fraction adds impetus to this issue, as the underlying mechanism of this syndrome appears to exhibit sex differences, and therapies are lacking ${ }^{4}$.

\section{Methods}

We performed a systematic review of the literature to quantify sex-specific reporting of ADRs of HF medications. HF medications recommended by the $2016 \mathrm{HF}$ guidelines from the European Society of Cardiology (ESC) ${ }^{4}$ were grouped into five groups:angiotensin-converting enzyme inhibitors, beta-blockers, angiotensin II receptor blockers, mineralocorticoid receptor antagonists and ivabradine ${ }^{4}$. Digoxin was included because of its suggested harmful effects in women'.

First, all clinical trials cited in the ESC $2016 \mathrm{HF}$ guidelines were extracted. Second, a systematic search of the website clinicaltrials.gov was performed on II November 2017. The search strategy included "Heart Failure" and the intervention options "ACE inhibitor", "Beta blocker", "Mineralocorticoid Receptor Antagonists", "Digoxin", "Ivabradine” and “Angiotensin II Receptor Blocker”. Records were excluded when:

(I) The study was ongoing, had been withdrawn or terminated, or had no published report available;

(2) The intervention was not part of one of the six drug families mentioned above;

(3) The primary study pouplation consisted of patients with comorbidites such as diabetes mellitus, chronic kidney disease, pulmonary hypertension, cancer, or Chagas disease;

(4) The outcome of interest was not (a) the incidence of cardiovascular disease, (b) hospitalisation for cardiac disease, or (c) (cardiovascular) mortality. 


\section{Results}

The ESC guidelines cited fifteen trials and two trial sub-studies, adding up to seventeen trials in total. The systematic search identified six additional trials from the 235 returned records, bringing the final study database to 23 trials. The main publication and any subsequent publications for each of these trials were screened for sex-specific efficacy and safety data. For all trials, data on inclusion and exclusion criteria, efficacy and safety of the medication, total number of participants, percentage of women, and age of the participants were extracted.

In total, the 23 identified trials included 10I,564 participants ( $25 \%$ women) with an average age of 65 years (59-76). The mean percentage of women enrolled across all trials was $25 \%$ (12-52\%). Fewer than half of the studies reported sex-specific efficacy data (I I/23, 48\%) and only two studies (9\%) presented sex-specific information about ADRs (Table I). In the SOLVD trial, the total number of ADRs reported compared to the total number of study participants was larger in women than in men in the enalapril group ( $35 \%$ vs $27 \%$ ) but similar in the placebo group (18\% vs $16 \%$ ). Women were overall more likely to report at least one ADR ( $26 \%$ vs $21 \%)$, but this difference was larger in the enalapril group (OR, I.30) than in the placebo group (OR, I.I5). Cough was reported by $3 \%$ of the placebo women and $10 \%$ of the women receiving enalapril, compared with $1.8 \%$ of the men on placebo (OR, I.67) and $4.2 \%$ of the men on enalapril (OR, 2.38) respectively. In the MERIT-HF trial, I0\% of men permanently stopped the study medication metoprolol because of an ADR compared with $8 \%$ of the women. These numbers were $12 \%$ and $10 \%$ respectively for the placebo group. The third largest study by study population and percentage of women $(7599,32 \%)$ did not report any sex-specific data, suggesting insufficient statistical power may not always explain the absence of sex-specific data (Table I).

\section{Discussion}

These data show sex-specific reporting of safety data is rare despite efforts to increase participation of women in cardiovascular trials ${ }^{3}$. The available sex-specific data suggest that women treated with enalapril may experience more ADRs while women treated with metoprolol were slightly less likely to permanently stop treatment due to ADRs than men. However, results based on 338 events in 1873 women are not sufficient to draw hard conclusions about the safety of these drugs for women. Reporting sex-stratified baseline data may help elucidate other differences between men and women that may explain the incidence of ADRs.

Next to sex-specific reporting, improvements in overall reporting of ADRs are needed. Under-reporting and variability in the level of report details are common in current reporting systems, which rely on investigators and clinicians to report events. The quality of ADR 
Table I | Overview of the trials included in the systematic review

\begin{tabular}{|c|c|c|c|}
\hline Drug class & Drug & Acronym & $\mathbf{n}$ \\
\hline \multirow[t]{4}{*}{ ACEI } & enalapril & CONSENSUS & 253 \\
\hline & & SOLVD Treatment & 2569 \\
\hline & & SOLVD Prevention & 4228 \\
\hline & lisinopril & ATLAS & 3164 \\
\hline \multirow[t]{5}{*}{ MRA } & eplerenone & EMPHASIS-HF & 2747 \\
\hline & & J-EMPHASIS-HF & 221 \\
\hline & & EPHESUS & 6632 \\
\hline & spironolactone & RALES & 1663 \\
\hline & & TOPCAT & 3445 \\
\hline \multirow[t]{6}{*}{ Beta-blocker } & bucindolol & BEST & 2708 \\
\hline & carvedilol & COPERNICUS & 2279 \\
\hline & & US Carvedilol Heart Failure Study & 1094 \\
\hline & metoprolol & MERIT-HF & 3991 \\
\hline & nevibilol & SENIORS & 2128 \\
\hline & bisoprolol & CIBIS II & 2647 \\
\hline \multirow[t]{3}{*}{ ARB } & candesartan & CHARM-Alternative & 2028 \\
\hline & & CHARM-Overall & 7599 \\
\hline & losartan high & HEAAL & 3834 \\
\hline Digitalis glycoside & digoxin & DIG & 6800 \\
\hline \multirow[t]{3}{*}{ If channel inhibitor } & ivabradine & SHIFT & 6505 \\
\hline & & BEAUTIFUL & 10917 \\
\hline & & SIGNIFY & 19102 \\
\hline
\end{tabular}

* Sex-specific adverse drug reaction data was available for the merged data from both SOLVD trials. These two trials were thus taken as one, bringing the total number of studies for the calculation of the prevalence of sex-specific reporting to 22 .

ACEI = Angiotensin-Converting Enzyme Inhibitors, ARB = Angiotensin II Receptor Blocker, ATLAS = Assessment of Treatment with Lisinopril and Survival, BEAUTIFUL = morBidity-mortality EvAlUaTion of the If inhibitor ivabradine in patients with coronary disease and left ventricULar dysfunction, BEST = Beta-blocker Evaluation of Survival Trial, CHARM = Candesartan in Heart failure Assesment of Reduction in Mortality and morbidity, CIBIS = Cardiac Insufficiency Bisoprolol Study, CONSENSUS = Cooperative North Scandinavian Enalapril Survival Study, COPERNICUS = Carvedilol Prospective Randomized Cumulative Survival, DIG = Digitalis Intervention Group, EF = Ejection Fraction, EMPHASIS-HF = Eplerenone in Mild Patients Hospitalization And Survlval Study in Heart Failure, EPHESUS = Eplerenone Post-Acute Myocardial Infarction Heart Failure Efficacy and Survival Study, HEAAL = Heart failure Endpoint evaluation of Angiotensin II Antagonist Losartan, J-EMPHASIS-HF = JapaneseEMPHASIS-HF, MERIT-HF = Metoprolol CR/XL Randomised Intervention Trial in Congestive Heart Failure, MRA $=$ Mineralocorticoid Receptor Antagonist, RALES = Randomized Aldactone Evaluation Study, SENIORS = Study of the Effects of Nebivolol Intervention on Outcomes and Rehospitalisation in Seniors with Heart Failure, SHIFT = Systolic Heart failure treatment with the If inhibitor ivabradine Trial, SIGNIFY = Study Assessing the MorbidityMortality Benefits of the If inhibitor Ivabradine in Patients with Coronary Artery Disease, SOLVD = Studies of Left Ventricular Dysfunction, TOPCAT = Treatment of Preserved Cardiac Function Heart Failure with an Aldosterone Antagonist, Val-HeFT = Valsartan Heart Failure Trial 


\begin{tabular}{|c|c|c|c|c|}
\hline $\begin{array}{l}\text { Women } \\
\text { (\%) }\end{array}$ & $\begin{array}{c}\text { Average age } \\
\text { (years) }\end{array}$ & $\begin{array}{c}\text { Sex-specific } \\
\text { adverse events }\end{array}$ & $\begin{array}{c}\text { Sex-specific } \\
\text { efficacy }\end{array}$ & $\begin{array}{l}\text { Inclusion: } \\
E F \leq 40 \%\end{array}$ \\
\hline 29.5 & 71 & no & no & not mentioned \\
\hline 19.7 & 61 & yes & no & yes \\
\hline 11.5 & 59 & yes & no & yes \\
\hline 20.5 & 64 & no & yes & yes \\
\hline 22.3 & 69 & no & no & yes \\
\hline 20.4 & 69 & no & no & yes \\
\hline 29.0 & 64 & no & no & yes \\
\hline 27.0 & 65 & no & no & yes \\
\hline 51.6 & 69 & yes & yes & no \\
\hline 22.0 & 60 & no & no & yes \\
\hline 20.5 & 63 & no & no & yes \\
\hline 23.4 & 58 & no & yes & yes \\
\hline 22.5 & 64 & no & yes & yes \\
\hline 36.9 & 76 & no & yes & yes \\
\hline 19.5 & 61 & no & yes & yes \\
\hline 31.9 & 67 & no & no & yes \\
\hline 31.6 & 66 & no & no & no \\
\hline 29.5 & 66 & no & yes & yes \\
\hline 22.4 & 63 & no & yes & no \\
\hline 23.5 & 60 & no & yes & yes \\
\hline 17.0 & 65 & no & yes & yes \\
\hline 27.6 & 65 & no & yes & no \\
\hline
\end{tabular}

reporting in clinical trials is not on par with the overall quality of clinical trial publications ${ }^{5}$, emphasising the importance of addressing this matter. Potential areas for improvement include stimulating patients and healthcare professionals to more actively report ADRs, the use of comprehensive algorithms for ADR reporting in clinical trials, and in appropriate settings, the use of patient-reported outcome tools designed for ADR reporting ${ }^{5}$.

While limited inclusion of women and the lack of reporting of sex-specific data may be two sides of the same coin and should be dealt with simultaneously, we argue that they should be tackled separately. Increasing the participation of women in clinical trials may not automatically improve sex-stratified reporting. We therefore argue that both proportionate representation of women in HF trials and sex-specific reporting of efficacy and safety data are of paramount importance for improving the quality of HF treatment. 


\section{Acknowledgements}

The authors wish to thank Cara Tannenbaum, Danielle Day, Paula A. Rochon, Vera RegitzZagrosek and Virginia M. Miller for their valuable input.

\section{Sources of funding}

This study was funded by the Dutch Heart Foundation (2013T084, Queen of Hearts Program)

2 and by ZonMw grant (849100003, Reviews en Kennissyntheses Gender en Gezondheid). 


\section{References}

I. Rosano GMC, Lewis B, Agewall S, et al. Gender differences in the effect of cardiovascular drugs: a position document of the Working Group on Pharmacology and Drug Therapy of the ESC. European heart journal 20I5; 36(40): 2677-80.

2. Leporini C, De Sarro G, Russo E. Adherence to therapy and adverse drug reactions: is there a link? Expert Opinion on Drug Safety 20I4; I3(sup I): 4I-55.

3. Melloni C, Berger JS, Wang TY, et al. Representation of women in randomized clinical trials of cardiovascular disease prevention. Circulation Cardiovascular quality and outcomes 2010; 3(2): I35-42.

4. Ponikowski P,Voors AA, Anker SD, et al. 2016 ESC Guidelines for the diagnosis and treatment of acute and chronic heart failure: The Task Force for the diagnosis and treatment of acute and chronic heart failure of the European Society of Cardiology (ESC). Developed with the special contribution of the Heart Failure Association (HFA) of the ESC. European journal of heart failure 2016; I8(8): 891-975.

5. Peron J, Maillet D, Gan HK, Chen EX, You B.Adherence to CONSORT adverse event reporting guidelines in randomized clinical trials evaluating systemic cancer therapy: a systematic review. J Clin Oncol 20I3; 3 I (3I): 3957-63. 



\section{Adverse drug reactions to guideline-recommended heart failure drugs in women: a systematic review of the literature}

3

Sophie H. Bots Floor Groepenhoff Anouk L.M. Eikendal

Cara Tannenbaum Paula A. Rochon Vera Regitz-Zagrosek Virginia M. Miller Danielle Day Folkert W.Asselbergs Hester M. den Ruijter 


\section{Abstract}

\section{Objectives}

To summarise all available evidence on sex differences in adverse drug reactions (ADRs) to heart failure (HF) medication.

\section{Background}

Women are more likely to experience ADRs than men, which may negatively affect their immediate and long-term health. HF in particular is associated with increased ADR risk due to the high number of comorbidities and older age. However, little is known about ADRs in female HF populations treated with guideline-recommended drugs.

\section{Methods}

We performed a systematic search in PubMed and Embase to collect all available information on ADRs to angiotensin-converting enzyme inhibitors (ACEls), beta-blockers, angiotensin II receptor blockers (ARBs), mineralocorticoid receptor antagonists (MRAs), ivabradine and digoxin in both women and men with HF.

\section{Results}

The search identified 155 eligible records, of which only II (7\%) reported ADR data for women and men separately. Sex-stratified reporting of ADRs did not increase over the last decades. Six of the II studies did not report sex differences. Three studies reported a higher risk of $A C E l$-related ADRs in women, one study showed higher digoxin-related mortality risk for women, and one study reported a higher risk of MRA-related ADRs in men. No sex differences in ADRs were reported for ARBs and beta-blockers. Sex-stratified data were not available for ivabradine.

\section{Conclusions}

These results underline the scarcity of ADR data stratified by sex. We call for a change in standard scientific practice towards reporting of ADR data for women and men separately. 


\section{Introduction}

Women have an approximately I.5 times higher risk of developing adverse drug reactions (ADRs) than men ${ }^{1,2}$. They are not only at a higher risk of hospitalisation due to the severity of their ADR but are also more likely to discontinue their treatment thereby losing its potential benefit ${ }^{1-4}$. A precise assessment of sex-specific ADRs is complicated by the rare reporting of such events in younger, predominantly male clinical trial populations with few co-morbidities ${ }^{5-7}$, as well as the lack of women in Phase I clinical trials that collect data on tolerability and dose-related $\mathrm{ADRs}^{8}$. As a result, it is unclear which ADRs to look for during post-marketing surveillance, a system that itself is also limited by high rates of underreporting and reporting bias?

The lack of sex-specific ADR data is especially pertinent in heart failure (HF) due to the high prevalence of comorbidities ${ }^{10}$ and polypharmacy in this population ${ }^{\prime \prime}$. Women with HF are less likely to receive guideline-recommended treatment ${ }^{12,13}$, possibly because of an increased risk for certain ADRs ${ }^{14}$. Due to the underrepresentation of women in all phases of clinical trials, little is known about female-specific ADRs in HF populations treated with guidelinerecommended drugs. To expand on an earlier effort evaluating sex-specific reporting in clinical trials ${ }^{15}$, we performed a systematic review to identify sex-specific ADRs to guidelinerecommended HF drugs.

\section{Methods}

Search strategy and selection criteria

We combined search results from PubMed, MEDLINE and the Embase database. Both databases were searched on 20 February 2018 using a pre-defined search strategy consisting of both text words and MeSH headings. The text words were limited to title and abstract only. We used the terms female, women, male, men, sex, gender for the sex-specific part of the search strategy, the terms heart failure, heart decompensation, cardiac decompensation for the HF domain, and the terms drug-related side effects and adverse reactions, side effect, adverse effect for the ADR component. We specifically excluded chemotherapy-induced HF and studies in children. We included all ejection fractions. The search was updated on 18 October 2018.

Guideline-recommended HF drugs were based on the $2016 \mathrm{HF}$ treatment guidelines from the European Society of Cardiology (ESC) ${ }^{16}$. There are five groups of HF drugs: angiotensinconverting enzyme inhibitors (ACEls), beta-blockers, angiotensin II receptor blockers (ARBs), mineralocorticoid receptor antagonists (MRAs) and ivabradine ${ }^{16}$. We added digoxin because of its suggested harmful effects in women'.

Only original research articles written in English or Dutch were considered for inclusion. Records were included if they mentioned any sex-specific ADRs related to one of the 
recommended HF drugs. We excluded studies with study populations too small for sexstratified analyses ( $\mathrm{n}<50$ patients), where the primary study population was not HF specific or had reduced left ventricular function due to a recent myocardial infarction. We excluded studies where the results could not be linked back to one specific drug or where the drug was administered intravenously. We excluded studies where the drug was administered only once to evaluate first-dose effects. Lastly, we excluded all studies for which the full text could not be retrieved.

For all included studies, the population size, percentage of women and mean age of the study population, the description of the ADR type(s) reported and the sex-specific ADR results were extracted. Meta-analysis of the results was not possible due to heterogeneity. The data is presented separately for each of the five drug categories.

\section{Results}

The search returned 9,424 unique articles, of which 356 were eligible for full-text screening. The majority of these studies was excluded due to the lack of sex-specific data $(n=144$, $40 \%$ ) or because the study design did not match our search criteria $(n=96,27 \%)$. Of the remaining articles $(n=116), 25$ did not provide ADR data, 19 were written in a different language, 13 were duplicates and for 48 the full text could not be located. Eleven articles met the inclusion criteria and were included in the analyses (Figure I). The articles were distributed relatively equally across the three decades in which they were published, showing no upward trend in sex-specific ADR reporting over time (Figure 2).

Importantly, these II studies comprised only seven percent of the 155 studies that reported ADR data. The II studies included 153,945 individuals with a mean age of 64 years (5275 years) and included on average $25 \%$ women (13\%-49\%), which was similar to the 144 excluded studies (29\%). Four studies (36\%) reported more ADRs in women compared with men, while one study $(9 \%)$ reported more ADRs in men. The remaining six studies (55\%) reported no difference in ADRs between the sexes. Six studies were post-hoc analyses from randomised clinical trials, two used data from healthcare insurance claims databases and the remaining three used patient cohorts from HF clinics (Table I). The availability of sex-specific ADR data varied across the different drug categories. Two out of seven digoxin studies reported sex-specific data (29\%), which decreased to one in eight for ACEI (5/40, I3\%) and even lower fractions for the other drugs. Sex-specific data were unavailable for ivabradine (Table I).

\section{Angiotensin-converting enzyme inhibitors}

There were 40 articles with ADR data for ACEls, of which five contained sex-specific ADR data (Table I). These five studies enrolled I37,956 patients with a mean age of 63 years (60-75 years) and on average $26 \%$ women. The Systems BIOlogy Study to TAilored 
Treatment in Chronic Heart Failure (BIOSTAT-CHF) study looked at three different HF drugs, including ACEls, bringing the total number of ACEI studies to six (Table I).

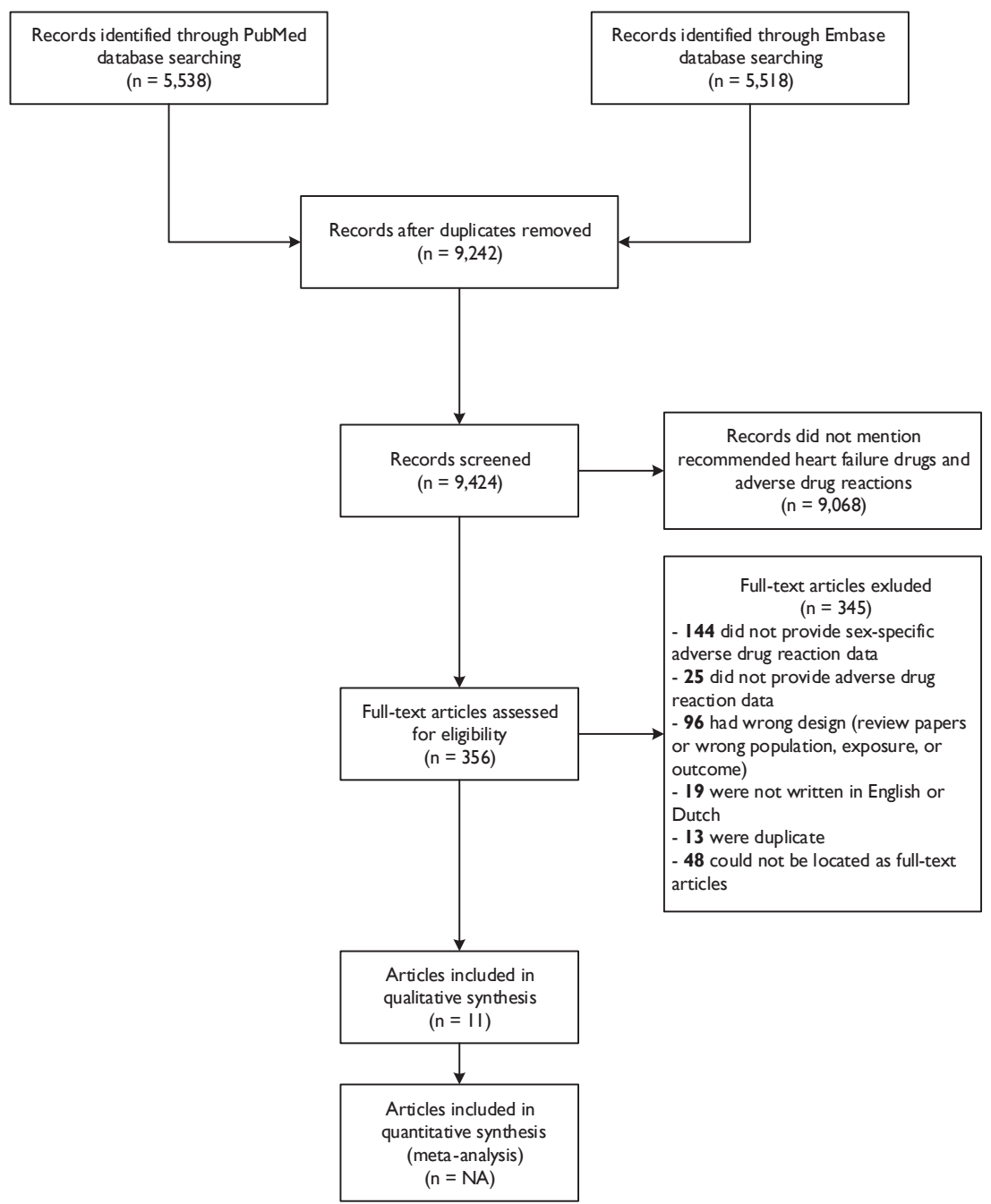

Figure I | Overview of the systematic search process

Data from an American claims database showed that the incidence of angio-oedema was 5.16 (3.37-7.92) per 1000 person-years in women who initiated ACEl treatment compared with 2.32 (1.48-3.64) per 1000 person-years in men ${ }^{17}$. Similarly, two post-hoc analyses of the Studies Of Left Ventricular Dysfunction (SOLVD) reported more ADRs in women than in men ${ }^{18,19}$. The difference was especially pronounced for cough, which was almost 2.5 times more prevalent in women compared with men ${ }^{18}$. However, this difference was not found in 
a Japanese hospital-based study, where the percentage of cough-related ADRs was similar between men and women ${ }^{20}$. A third post-hoc analysis of the SOLVD trial showed that a similar percentage of men and women experienced at least one episode of anaemia during enalapril treatment $(38 \% \text { versus } 41 \%)^{21}$. Similarly, a post-hoc analysis of the BIOSTAT-CHF study found no significant difference in the number of men and women who failed to reach the target dose of ACEl or ARB due to ADRs (25\% versus $27 \%)^{22}$.

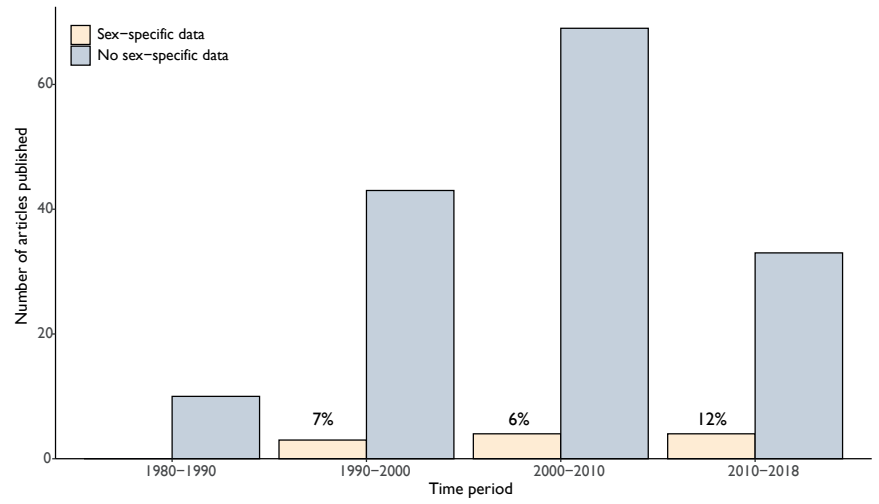

Figure 2 | Sex-specific ADR reporting across the last four decades

\section{Angiotensin II receptor blockers}

The search returned 23 articles with ADR data for ARBs, of which one contained sexspecific ADR data (Table I). The BIOSTAT-CHF study also evaluated ARBs, bringing the total number of sex-specific ARB studies to two. A post-hoc analysis of the Heart failure Endpoint evaluation of Angiotensin II Antagonist Losartan (HEAAL) study, which enrolled 3,834 participants with a mean age of 67 years and $29 \%$ women, reported that there were no significant differences in risk of kidney impairment, hyperkalaemia or hypotension between men and women treated with losartan ${ }^{23}$ (Table I). As mentioned above, a similar percentage of men and women failed to reach the target dose of ARB or ACEI in the BIOSTAT-CHF study ${ }^{22}$.

\section{Beta-blockers}

In total 45 articles provided ADR data for beta-blockers, of which one reported sex-specific ADR data (Table I).The BIOSTAT-CHF study included an evaluation of beta-blockers, bringing the total number of beta-blocker studies to two. A study from an HF clinic in Australia, which included $230 \mathrm{HF}$ patients with a mean age of 52 years and I $3 \%$ women, reported that men and women treated with carvedilol reported similar numbers of ADRs ( $12 \%$ vs $10 \%$, respectively) ${ }^{24}$. Data from the BIOSTAT-CHF study suggest that a similar percentage of men and women failed to reach target dose due to ADRs ( $20 \%$ vs $22 \%$, respectively $)^{22}$. 


\section{Digitalis glycosides}

There were seven articles with ADR data for digoxin, of which two evaluated the effects of sex (Table I). Together these two studies included 9,69I patients with a mean age of 67 years (65-70 years) and on average $28 \%$ women (Table I). A post-hoc analyses of the Digitalis Intervention Group (DIG) data suggested that women treated with digoxin had an approximately $20 \%$ higher risk of death compared with the placebo group $(H R=1.23$, 95\%Cl: I.02-I.47) while this difference was not seen for men $(\mathrm{HR}=0.93,95 \% \mathrm{Cl}:-.85-1.02)^{25}$. This sex difference was not present in data from an American claims cohort, where the risk for death and hospitalisation was similar for men and women ${ }^{26}$.

\section{Mineralocorticoid receptor antagonists}

The search returned 18 articles with ADR data for MRAs, of which one reported sexspecific ADR data (Table I). This study enrolled I $34 \mathrm{HF}$ patients with a mean age of 66 years and $31 \%$ women. The patients were followed up for discontinuation of treatment due to hyperkalaemia, deterioration of renal function based on serum creatinine, and gynecomastia in men. They found that $16 \%$ of the women treated with spironolactone withdrew from treatment due to ADRs compared with $28 \%$ of the men ${ }^{27}$.

\section{Ivabradine}

In total three studies provided ADR data for ivabradine, none of which reported sex-specific results (Table I).

\section{Discussion}

We show a general lack of information about sex-specific ADRs for guideline-recommended HF drugs. Of the I55 ADR records returned by the search, only II (7\%) provided sex-specific ADR data. The majority of these II studies (55\%) reported no sex differences in ADRs. Women may have more ADRs related to ACEls and digoxin, while men may experience more ADRs related to MRAs. However, the low number of studies and participants in some studies make it difficult to draw solid conclusions.

\section{Lack of sex-specific data}

We show that the lack of sex-specific ADR data is widespread in observational studies. Only $7 \%$ of all available studies, spanning a large range of study population sizes and publication years, reported sex-specific ADR results. In line with the limited effect of efforts to increase the participation of women in cardiovascular trials ${ }^{28}$, there was no upward trend in sexspecific reporting over time. We therefore argue that sex-specific reporting should receive attention separately from the proportionate representation of women, despite these problems being connected. 


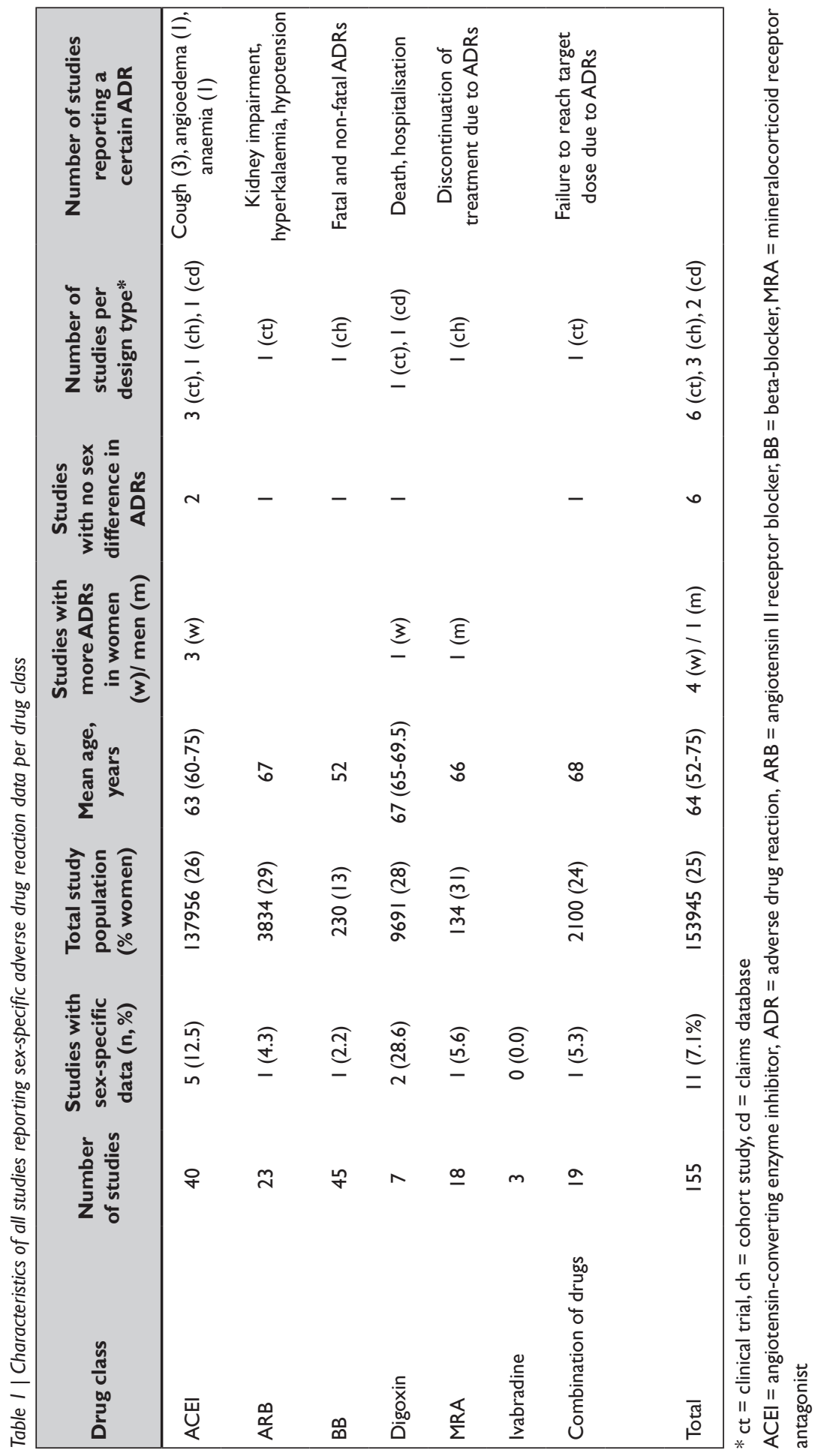


Sex-specific data reporting should be regarded as standard practice instead of a statistical power-dependent subgroup analysis. Reporting data for women and men separately reflects proper scientific conduct to support future meta-analyses. In situations where data are scarce even the smallest studies can contribute, an argument also made for dementia trials ${ }^{29}$.

\section{Underrepresentation}

The persistent underrepresentation of women in clinical trials ${ }^{6,28,30-32}$ calls for a new approach to address the lack of female-specific data (Central Illustration). ADRs that might be relatively common in women become too rare to be detected in a clinical trial population with only few women, creating an evidence gap. In addition, the lack of sex-stratified data hinders the identification of sex-specific ADR trends. Observational studies have the unique potential to fill this evidence gap because they include more women and are thus able to stratify their results by sex. Early-stage safety and dose-finding trials should also be included in this effort because they have the opportunity to detect sex differences early on without the need to conduct large-scale studies. Observations from these studies can lead to interesting insights ${ }^{14}$ that can inform healthcare professionals and treatment guidelines on optimal treatment for both sexes until sufficient clinical trials with a proportionate amount of women and sexstratified ADR data have been conducted.

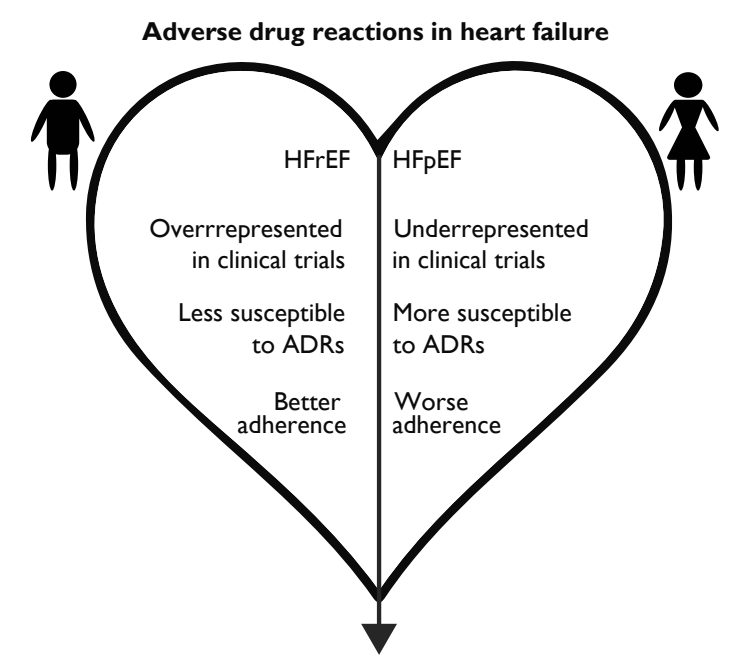

Report adverse drug reactions for women and men separately

Central Figure | Underlying sex differences in heart failure that necessitate sex-specific reporting

Susceptibility to ADRs

Patients suffering from HF often also have five or more comorbidities and take on average 10 different medications ${ }^{11,33,34}$. Women more often have HF with preserved ejection fraction 
(HFpEF) than men (Central Illustration), a subtype characterised by additional comorbidites and older age compared to other HF subtypes ${ }^{10,33}$. In addition, women seem to use more medications than men ${ }^{4,35}$. These factors increase the risk for drug-drug interaction ADRs in women with HF, which is indeed one of the three driving factors behind sex differences in ADR reporting ${ }^{2}$ (Central Illustration). The other two are sex differences in pharmacokinetics and pharmacodynamics ${ }^{2}$, of which differences in distribution volume, hepatic and renal clearance, and sex hormones seem to be the key players. The biological processes underlying these differences have been discussed in detail elsewhere ${ }^{36,37}$.

\section{Adherence and quality of life}

Interestingly, women with HFpEF report a poorer Quality of Life (QoL) than men regardless of disease severity ${ }^{38,39}$. Women report lower QoL due to worsening symptoms or decreased physical functioning and overall health, among others ${ }^{40}$. This may be induced by ADRs directly or indirectly via poor adherence, the latter being supported by the observations that QoL is positively related with adherence ${ }^{41}$ and that women are more likely to be poor adherers ${ }^{42}$. However, much is still unclear about sex differences in QoL ${ }^{40}$ and further research is needed to properly evaluate sex-specific effects of ADRs on QoL.

\section{Sex differences in adverse drug reactions}

Three out of the six ACEl articles included in our review suggested that women were more likely to experience ACEl-induced ADRs, while the other three showed no sex differences. The higher incidence of ACEl-induced cough in women has been observed previously ${ }^{43-45}$. In addition, $A R B s$ seem to have better efficacy and adherence profiles than ACEls in women with CHF but not in men ${ }^{14}$. Women may thus be at higher risk of ACEl-induced ADRs, which negatively affects their adherence and treatment benefit, but more data is needed to draw a solid conclusion. The small Japanese study identified by our search reported no sex differences in ACEl-induced ADRs while other studies suggest these ADRs to be more prevalent among Asian populations ${ }^{46,47}$. This finding may be explained by the small study size in combination with the small number of reported ADRs ( $n=37$, 19 women), or the sex difference may be masked by the higher ADR prevalence. Regarding angio-oedema the results are less clear, with some previous studies showing a higher incidence in women ${ }^{48}$ and others showing no sex differences ${ }^{49}$. Additional data on angio-oedema may create more insight into this matter.

We did not find evidence for sex differences in ADRs for ARBs and beta-blockers. Our results on digoxin are contradictory, as the higher risk of hospitalisation and death in women related to digoxin treatment observed in a post-hoc analysis from the DIG trial was not observed in a large cohort. Similarly, data from a British cohort study did not observe any sex differences in the risk of all-cause mortality treated with digoxin ${ }^{50}$ and there is some evidence that digoxin is equally beneficial in both men at women at low blood 
concentrations ${ }^{51}$. Scientific evidence claiming no sex differences may outweigh the evidence that suggests sex differences in digoxin-related ADRs are present. More data are needed to support this claim.

The only MRA article returned by our search showed a higher number of spironolactonerelated ADRs in men than women. Spironolactone is known to induce gynaecomastia ${ }^{52}$ and hyperkalaemia, which occur more frequently in men than women ${ }^{53}$. This might explain why men more often withdrew from MRA treatment than women, but additional data may shed more light on the issue.

\section{Strengths and limitations}

This systematic review combines all available knowledge on sex-specific ADRs for guidelinerecommended HF drugs. Due to lack of data and heterogeneity of the available data, the results could not be meta-analysed. The definition of HF was not identical across included studies, possibly leading to some misclassification in individual studies. As a result, we were unable to split our result by HF subtype. We excluded diuretics and sacubitril/valsartan from our search because the first are only used to treat symptoms and the second were discovered too recently for sex-specific post-hoc studies to be published but should be included in future efforts. The low number of returned studies obliges us to interpret our results with care. We were unable to discuss sex-specific ADRs for ivabradine due to lack of data. However, the scarcity of data in itself is an important finding that hopefully inspires future researchers to sex-stratify their results.

\section{Conclusion}

The scarcity of sex-specific ADR data for guideline-recommended HF drugs data hampers the identification of female-specific ADRs. The currently available evidence hints at the existence of sex-specific ADRs but remains inconclusive due to the scarcity of data. Sexspecific ADR reporting in articles has not increased over the past three decades. A call of action is needed to incorporate sex-specific reporting into scientific practice.

\section{Funding}

This study was funded by the Dutch Heart Foundation (2013T084, Queen of Hearts Program) and by ZonMw grant (849100003, Reviews en Kennissyntheses Gender en Gezondheid). Folkert Asselbergs is supported by UCL Hospitals NIHR Biomedical Research Centre. 


\section{References}

I. Rosano GMC, Lewis B, Agewall S, et al. Gender differences in the effect of cardiovascular drugs: a position document of the Working Group on Pharmacology and Drug Therapy of the ESC. European heart journal 20I5; 36(40): 2677-80.

2. Soldin OP, Mattison DR. Sex differences in pharmacokinetics and pharmacodynamics. Clinical pharmacokinetics 2009; 48(3): I43-57.

3. Leporini C, De Sarro G, Russo E. Adherence to therapy and adverse drug reactions: is there a link? Expert Opinion on Drug Safety 20I4; I3(supl): 4I-55.

4. Zopf Y, Rabe C, Neubert A, et al. Women encounter ADRs more often than do men. European journal of clinical pharmacology 2008; 64(I0): 999-1004.

5. Melloni C, Berger JS, Wang TY, et al. Representation of women in randomized clinical trials of cardio-vascular disease prevention. Circulation: Cardiovascular Quality and Outcomes 20 I0: CIRCOUTCOMES. I I 0.868307.

6. Scott PE, Unger EF, Jenkins MR, et al. Participation of Women in Clinical Trials Supporting FDA Approval of Cardiovascular Drugs. Journal of the American College of Cardiology 20 I8; 7I(I8): 1960-9.

7. Tahhan AS, Vaduganathan M, Greene SJ, et al. Enrollment of Older Patients, Women, and Racial and Ethnic Minorities in Contemporary Heart Failure Clinical Trials:A Systematic Review. JAMA cardiology 2018.

8. Yang Y, Carlin A, Faustino P, et al. Participation of Women in Clinical Trials for New Drugs Approved by the Food and Drug Administration in 2000-2002; 2009.

9. Hazell L, Shakir SAW. Under-Reporting of Adverse Drug Reactions. Drug Safety 2006; 29(5): 385-96.

10. Chamberlain AM, Sauver JLS, Gerber Y, et al. Multimorbidity in heart failure: a community perspective. The American journal of medicine 2015; I28(I): 38-45.

II. Mastromarino V, Casenghi M, Testa M, et al. Polypharmacy in Heart Failure Patients Current Heart Failure Reports 20I4; II(2): 2I2-9.

12. Crousillat DR, Ibrahim NE. Sex Differences in the Management of Advanced Heart Failure. Current Treatment Options in Cardiovascular Medicine 20I8; 20(II): 88.

13. Mehta PA, Cowie MR. Gender and heart failure: a population perspective. Heart (British Cardiac Society) 2006; 92 Suppl 3(Suppl 3): iiil4-iii8.

14. Hudson M, Rahme E, Behlouli H, Sheppard R, Pilote L. Sex differences in the effectiveness of angiotensin receptor blockers and angiotensin converting enzyme inhibitors in patients with congestive heart failure - A population study. European Journal of Heart Failure 2007; 9(6-7): 602-9.

15. Bots SH, den Ruijter HM. Recommended Heart Failure Medications and Adverse Drug Reactions in Women. Circulation 2019; I39(12): 1469-7I.

16. Ponikowski P,Voors AA, Anker SD, et al. 2016 ESC Guidelines for the diagnosis and treatment of acute and chronic heart failure: The Task Force for the diagnosis and treatment of acute and chronic heart failure of the European Society of Cardiology (ESC) Developed with the special contribution of the Heart Failure Association (HFA) of the ESC. European heart journal 2016; 37(27): 2129-200.

17. Do TP, Seetasith A, Belleli R, et al.A Database Cohort Study to Assess the Risk of Angioedema Among Patients with Heart Failure Initiating Angiotensin-Converting Enzyme Inhibitors in the USA. American Journal of Cardiovascular Drugs 2018; 18(3):205-II.

18. Kostis JB, Shelton B, Gosselin G, et al.Adverse effects of enalapril in the Studies of Left Ventricular Dysfunction (SOLVD). American heart journal 1996; I3I (2): 350-5.

19. Kostis JB, Shelton BJ,Yusuf S, et al.Tolerability of enalapril initiation by patients with left ventricular dysfunction: results of the medication challenge phase of the Studies of Left Ventricular Dysfunction. American heart journal 1994; 128(2): 358-64.

20. Sadanaga T, Yoshimura M, Sakamoto T, Sumida H, Ogawa H. Enalapril-induced cough is associated with nonsevere heart failure. International journal of cardiology 2009; 135(2):275-6.

2I. Ishani A, Weinhandl E, Zhao Z, et al. Angiotensin-converting enzyme inhibitor as a risk factor for the development of anemia, and the impact of incident anemia on mortality in patients with left ventricular dysfunction. Journal of the American College of Cardiology 2005; 45(3): 391 -9. 
22. Ouwerkerk W, Voors A, Anker S, et al. Determinants and clinical outcome of uptitration of ACE-inhibitors and beta-blockers in patients with heart failure: a prospective European study. European heart journal 2017; 38(24): I883-90.

23. Kiernan MS, Wentworth D, Francis G, et al. Predicting adverse events during angiotensin receptor blocker treatment in heart failure: results from the HEAAL trial. European journal of heart failure 20 I2; I $4($ I 2): I 40 I9.

24. Macdonald PS, Keogh AM,Aboyoun CL, Lund M,Amor R, McCaffrey DJ.Tolerability and efficacy of carvedilol in patients with New York HeartAssociation class IV heart failure Journal of the American College of Cardiology 1999; 33(4): 924-31.

25. Rathore SS, Wang Y, Krumholz HM. Sex-based differences in the effect of digoxin for the treatment of heart failure. New England Journal of Medicine 2002; 347(18): I403-II.

26. Freeman JV, Yang J, Sung SH, Hlatky MA, Go AS. Effectiveness and safety of digoxin among contemporary adults with incident systolic heart failure. Circulation:Cardiovascular Quality and Outcomes 2013: CIRCOUTCOMES. II I.000079.

27. Lopes RJ, Lourenço AP, Mascarenhas J, Azevedo A, Bettencourt P. Safety of spironolactone use in ambulatory heart failure patients. Clinical Cardiology:An International Indexed and Peer-Reviewed Journal for Advances in the Treatment of Cardiovascular Disease 2008;3 I ( I I): 509-I3.

28. Pilote L, Raparelli V. Participation of Women in Clinical Trials: Not Yet Time to Rest on Our Laurels. Elsevier; 2018.

29. Mehta N, Rodrigues C, Lamba M, et al. Systematic Review of Sex-Specific Reporting of Data: Cholinesterase Inhibitor Example. Journal of the American Geriatrics Society 2017; 65(I0): 22I 3-9.

30. Kim ES, Carrigan TP, Menon V. Enrollment of women in National Heart, Lung, and Blood Institute-funded cardiovascular randomized controlled trials fails to meet current federal mandates for inclusion. Journal of the American College of Cardiology 2008; 52(8): 672-3.

31. Levinsson A, Dubé MP, Tardif JC, de Denus S. Sex, drugs, and heart failure: a sex-sensitive review of the evidence base behind current heart failure clinical guidelines. ESC heart failure 2018.

32. Rochon PA, Clark JP, Binns MA, Patel V, Gurwitz JH. Reporting of gender-related information in clinical trials of drug therapy for myocardial infarction. Canadian Medical Association Journal 1998; I59(4): 32 I-7.

33. Dunlay SM, Roger VL, Redfield MM. Epidemiology of heart failure with preserved ejection fraction. Nature Reviews Cardiology 2017; I4:591.

34. Baron-Franco B, McLean G, Mair FS, Roger VL, Guthrie B, Mercer SW. Comorbidity and polypharmacy in chronic heart failure: a large cross-sectional study in primary care. The British journal of general practice : the journal of the Royal College of General Practitioners 2017; 67(658): e3 I4-e20.

35. Perry BA,Turner LW.A prediction model for polypharmacy: are older, educated women more susceptible to an adverse drug event? Journal of women \& aging 200I; I3(4): 39-5I.

36. Soldin OP, Chung SH, Mattison DR. Sex differences in drug disposition. Journal of biomedicine \& biotechnology 20II; 20II: I87I03-.

37. Nicolson TJ, Mellor HR, Roberts RRA. Gender differences in drug toxicity. Trends in Pharmacological Sciences 2010; $31(3)$ : 108-I4.

38. Faxen UL, Hage C, Donal E, Daubert JC, Linde C, Lund LH. Patient reported outcome in HFpEF: Sex-specific differences in quality of life and association with outcome. Int J Cardiol 20I 8; 267: I28-32.

39. Lewis EF, Lamas GA, O'Meara E, et al. Characterization of health-related quality of life in heart failure patients with preserved versus low ejection fraction in CHARM. European Journal of Heart Failure 2007; 9(I): 83-9I.

40. Johansson P, Dahlström U, Broström A. Factors and Interventions Influencing Health-Related Quality of Life in Patients with Heart Failure:A Review of the Literature. European Journal of Cardiovascular Nursing 2006; 5(I): 5-I5.

4I. Silavanich V, Nathisuwan S, Phrommintikul A, Permsuwan U. Relationship of medication adherence and quality of life among heart failure patients. Heart \& Lung 2018. 
42. Granger BB, Swedberg K, Ekman I, et al. Adherence to candesartan and placebo and outcomes in chronic heart failure in the CHARM programme: double-blind, randomised, controlled clinical trial. The Lancet 2005; 366(9502): 2005-II.

43. Gibson GR. Enalapril-induced cough. Archives of Internal Medicine 1989; I49(I2): 270 I-3.

44. Yesil S, Yesil M, Bayata S, Postaci N.ACE inhibitors and cough. Angiology 1994; 45(9): 805-8.

45. Coulter DM, Edwards IR. Cough associated with captopril and enalapril. British medical journal (Clinical research ed) 1987; 294(6586): I52I-3.

46. Lee YJ, Tsai JC. Angiotensin-converting enzyme gene insertion/deletion, not bradykinin B2 receptor-58T/C gene polymorphism, associated with angiotensin-converting enzyme inhibitor-related cough in Chinese female patients with non-insulin-dependent diabetes mellitus. Metabolism: clinical and experimental 200I; 50(I I): I346-50.

47. Woo KS, Nicholls MG. High prevalence of persistent cough with angiotensin converting enzyme inhibitors in Chinese. Br J Clin Pharmacol 1995; 40(2): I4I-4.

48. Miller DR, Oliveria SA, Berlowitz DR, Fincke BG, Stang P, Lillienfeld DE.Angioedema Incidence in USVeterans Initiating Angiotensin-Converting Enzyme Inhibitors. Hypertension 2008; 5 I (6): I624-30.

49. Banerji A, Blumenthal KG, Lai KH, Zhou L. Epidemiology of ACE Inhibitor Angioedema Utilizing a Large Electronic Health Record. The journal of allergy and clinical immunology In practice 2017; 5(3): 744-9.

50. Flory JH, Ky B, Haynes K, et al. Observational cohort study of the safety of digoxin use in women with heart failure. BMJ Open 20I2; 2(2).

5I. Ahmed A, Aban IB, Weaver MT, Aronow WS, Fleg JL. Serum digoxin concentration and outcomes in women with heart failure: A bi-directional effect and a possible effect modification by ejection fraction. European journal of heart failure 2006; 8(4): 409-19.

52. Narula HS, Carlson HE. Gynaecomastia-pathophysiology, diagnosis and treatment. Nature Reviews Endocrinology 2014; 10:684.

53. DeFilippis EM, Desai AS. Treatment of Hyperkalemia in Heart Failure. Current Heart Failure Reports 2017; I4(4): 266-74. 
ADRS TO GUIDELINE-RECOMMENDED HF DRUGS 



\title{
Routine clinical care data
} from thirteen cardiac outpatient clinics - design of the Cardiology Centers of the Netherlands (CCN) database

\author{
Sophie H. Bots \\ Klaske R. Siegersma \\ N. Charlotte Onland-Moret \\ Folkert W.Asselbergs \\ G.Aernout Somsen \\ Igor I.Tulevski \\ Hester M. den Ruijter \\ Leonard Hofstra
}




\begin{abstract}
Background

Despite the increasing availability of clinical data due to the digitalisation of healthcare systems, data often remain inaccessible due to the diversity of data collection systems. In the Netherlands, Cardiology Centers of the Netherlands (CCN) introduced "one-stop shop" diagnostic clinics for patients suspected of cardiac disease by their general practitioner. All $\mathrm{CCN}$ clinics use the same data collection system and standardised protocol, creating a large regular care database. This database can be used to describe referral practices, evaluate risk factors for cardiovascular disease (CVD) in important patient subgroups, and develop prediction models for use in daily care.
\end{abstract}

\title{
Construction and Content
}

The current database contains data on all patients who underwent a cardiac workup in one of the 13 CCN clinics between 2007 and February 2018 ( $n=109,151,52 \%$ women). Data were pseudonymised and contain information on anthropometrics, cardiac symptoms, risk factors, comorbidities, cardiovascular and family history, standard blood laboratory measurements, transthoracic echocardiography, electrocardiography in rest and during exercise, and medication use. Clinical follow-up is based on medical need and consisted of either a repeat visit at CCN (44\%) or referral for an external procedure in a hospital (I7\%). Passive follow-up via linkage to national mortality registry is available for $96 \%$ of the database.

\section{Utility and Discussion}

The CCN database provides a strong base for research into historically underrepresented patient groups due to the large number of patients and the lack of in- and exclusion criteria. It also enables the development of artificial intelligence-based decision support tools. Its contemporary nature allows for comparison of daily care with the current guidelines and protocols. Missing data is an inherent limitation, as the cardiologist could deviate from standardised protocols when clinically indicated.

\section{Conclusion}

The CCN database offers the opportunity to conduct research in a unique population referred by their general practitioner to the cardiologist for diagnostic workup. Together with its large size, the representation of historically underrepresented patient groups and contemporary nature, this makes it a valuable tool for expanding our knowledge of cardiovascular diseases. 


\section{Introduction}

Cardiovascular diseases (CVD) remain an important cause of death and disability worldwide ${ }^{1,2}$. The digitalisation of the healthcare system has made a wealth of clinical care data available for researchers ${ }^{3-6}$. This provides a unique opportunity for researchers to evaluate pressing topics in cardiovascular medicine. The added value of clinical care data in cardiovascular research is threefold. First, clinical care data better reflect the current real-world situation in healthcare with regard to clinical presentation of disease and representation of patient groups. This is especially relevant for patient groups that have historically been underrepresented in clinical studies such as women ${ }^{7}$, the elderly ${ }^{8}$, and patients with multimorbidity ${ }^{9}$ CVD in women may be different from CVD in men in several aspects, including the clinical presentation, the effect of traditional risk factors and presence of female-specific risk factors related to pregnancy and menopause, and the efficacy of treatment ${ }^{10}$. Elderly patients and those with multimorbidity also need to be studied to combat the rising prevalence of CVD risk factors such as hypertension, diabetes and obesity ${ }^{11,12}$. Second, clinical care data contain a large number of individuals and wide range of clinical measurements, a combination that is difficult to obtain within a research setting. This facilitates the development of prediction models and decision support tools using artificial intelligence methods that can subsequently be implemented within the healthcare system. These tools can help healthcare professionals to interpret large amounts of patient data and assist healthcare decision-making. Third, researchers can use clinical care data to evaluate the current state of clinical practice, adherence to guidelines, and develop treatment and referral strategies that better suit the current presentation of patients suspected of CVD.

However, data from earlier stages in the clinical care pathway remain difficult to access due to the smaller size of single general practitioner (GP) offices and the diversity of data collection systems. To close this gap, a collaboration was set up between the University Medical Center Utrecht (UMCU) and Cardiology Centers of the Netherlands (CCN), an organisation of I 3 cardiac outpatient clinics that operate between the GP and the hospital cardiologist. In the Netherlands, CCN introduced “one-stop shop" cardiac outpatient clinics to facilitate efficient diagnostic workup for cardiac disease and fast diagnosis of potential life-threatening pathologies. GPs can refer their patients to a CCN clinic for cardiac workup when they suspect their patient suffers from cardiac disease. All CCN clinics perform the same standardised protocol and store their data in a shared data collection system. Follow-up appointments and results from referrals for advanced cardiac imaging or cardiac interventions are stored in the same system. As a result of this set-up, CCN offers a unique opportunity to obtain semi-structured data on a large group of patients at an early stage of the regular care pathway.

The aim of this paper is to describe the CCN clinical care database. The database contains data on a large number of individual patients and a wide range of standardised characteristics 
from a unique population situated between the GP and the hospital cardiologist. The clinical nature of the database ensures that it reflects the patient population currently seen in daily care, including those that may be underrepresented in clinical research. The database can be used to describe current clinical practice, evaluate the prevalence of cardiovascular risk factors and their relation to cardiovascular disease, and develop prediction algorithms that have the potential to be implemented in daily care.

\section{Construction and content}

\section{Data generation at CCN clinics}

\section{Baseline examination}

Every patient referred to one of the $\mathrm{CCN}$ clinics underwent a standardised diagnostic workup. This protocol consisted of transthoracic echocardiography (TTE) and ultrasound imaging of the carotid arteries, electrocardiography at rest (ECG) and during exercise (stress ECG), a laboratory test, and a consult with a nurse during which self-reported anthropometrics, symptoms, cardiovascular risk factors, and comorbidities were registered. Past medication use and cardiovascular history were also recorded, as well as on-site clinical diagnoses made by the cardiologist. An overview of all the stored clinical characteristics can be found in Table I.

Body mass index was calculated based on self-reported height and weight. Blood pressure was measured with a Microlife WatchBP. TTE was performed with a General Electric Vivid E6 or E7 echocardiography device. Blood samples were analysed with the Roche Reflotron Sprint system. The ECG was recorded with the Welch Allyn Cardioperfect Pro recorder in supine position with 12 leads. The stress ECG was performed on a watt bike from Lode Corival Eccentric with simultaneous blood pressure measurements (Medtronic BL-6 Compact) and ECG recording (Welch Allyn Cardioperfect recorder). Raw data of the ECG, stress ECG and TTE were not available. Medication and diagnoses were recorded as semistructured text.

While CCN has standardised and uniform diagnostic workup protocols for every patient, in practice a cardiologist may deviate from this protocol when this is clinically indicated. For example, the cardiologist may choose not to perform a stress ECG in patients with a contraindication to the procedure, such as very high systolic blood pressure ${ }^{13}$. This introduces missing data, illustrated by the baseline stress ECG data which were missing for $25 \%$ of patients in the CCN database (Figure I). 
Table I | Overview of all features stored in the database

\begin{tabular}{|c|c|}
\hline Phase & Measurement \\
\hline \multirow{39}{*}{$\begin{array}{l}\text { Baseline } \\
(2007-F e b 2018)\end{array}$} & Consult \\
\hline & $\begin{array}{l}\text { (-) Presence and characteristics of cardiac symptoms (chest pain, dyspnoea, fatigue, } \\
\text { palpitations, collapse, heart murmurs) }\end{array}$ \\
\hline & (-) Anthropometrics (height, weight, hip circumference, blood pressure, heart rate, heart \\
\hline & and breathing sounds, pulse, palpation) \\
\hline & Intake \\
\hline & (-) Behavioural cardiovascular risk factors (smoking, alcohol use) \\
\hline & (-) Comorbidities (diabetes mellitus, hypertension, dyslipidaemia) \\
\hline & (-) Family history of cardiovascular disease (atherosclerosis, sudden death, \\
\hline & cardiomyopathy, arrhythmia) \\
\hline & Lab \\
\hline & (-) Lipids (total, high density, and low density cholesterol, triglycerides) \\
\hline & (-) Potassium, sodium, haemoglobin, glucose \\
\hline & (-) Glomerular filtration rate \\
\hline & (-) Lipoprotein A, brain natriuretic protein, thyroid stimulation hormone \\
\hline & TTE \\
\hline & (-) M-mode (dimensions of aorta and left heart chambers) \\
\hline & (-) Two-dimensional (evaluation of function and shape of all heart chambers and valves) \\
\hline & (-) Colour Doppler (valve insufficiencies and septum defects) \\
\hline & (-) Spectral Doppler (left ventricular diastolic function and gradients over valves) \\
\hline & (-) Intima media thickness (left and right, anterior and posterior) \\
\hline & ECG; \\
\hline & (-) Duration of defined ECG intervals and complexes (RR, PR, QRS, QT) \\
\hline & (-) ST depression, elevation, negative T-top, QRS axis \\
\hline & (-) Dilatation of left and right atrium, intraventricular conduction delay, left ventricular \\
\hline & 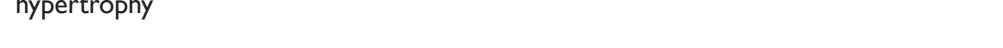 \\
\hline & Stress ECG; \\
\hline & (-) Protocol, device, target heart rate, use of beta-blocker before test \\
\hline & (-) ECG characteristics, blood pressure and heart rate before and during exercise test \\
\hline & (-) Duration and load of exercise test, exercise tolerance, reason to stop exercise test \\
\hline & (-) Arrhythmia or angina symptoms during exercise test, left ventricular hypertrophy \\
\hline & Decursus; \\
\hline & (-) Cardiologist summary of visit (free text) \\
\hline & Medication; \\
\hline & (-) Cardiovascular medication use grouped by researchers \\
\hline & (-) Date medication was started and date it was ended when applicable \\
\hline & Diagnosis; \\
\hline & (-) Cardiovascular diagnosis defined by researchers \\
\hline & (-) Cardiovascular risk factor diagnosis defined by researchers \\
\hline & (-) Date of diagnosis \\
\hline
\end{tabular}




\begin{tabular}{|ll|}
\hline Phase & Measurement \\
\hline $\begin{array}{l}\text { Follow-up } \\
(2007 \text { - Feb 20I8) }\end{array}$ & Consult, intake, lab, TTE, ECG, stress ECG and decursus as described for baseline \\
& External procedures; \\
& $(-)$ External procedure performed and location where it was performed \\
& $(-)$ External procedure grouped by researchers \\
& $(-)$ Date of appointment \\
& \\
Record linkage (2019) & All-cause mortality \\
& Education level \\
& Ethnicity \\
Personal income & Cause-specific mortality \\
\hline
\end{tabular}

Information collected during a patient's clinical trajectory within CCN

After the first visit, patients may enter a clinical trajectory during which one or more return visits to a CCN clinic are planned. Information collected during these clinical follow-up visits was also stored in the $\mathrm{CCN}$ database. This clinical follow-up was not standardised but rather based on medical need. As a result, clinical follow-up varies across patients in frequency, duration, and measurements obtained. During these clinical follow-up visits either all or some components of the standard screening protocol were repeated, with rest ECG being repeated most frequently (Figure I).

Patients in need of additional imaging or cardiac intervention based on the result of their initial CCN workup were referred to a nearby hospital as these facilities were not available at the $\mathrm{CCN}$ clinics. The referral itself and the summarised text results of these procedures were stored in the CCN database (Table I). Computed Tomography (CT) scans were performed most often, comprising $30.8 \%$ of all external procedures. The five most common external procedures can be found in Figure I.

\section{Database construction}

\section{Data extraction, cleaning and storage}

We extracted all data generated by CCN up to February 2018 from their data collection system. These raw files were cleaned and processed using SAS (SAS Institute Inc., North Carolina, USA) to create a relational database. This process included separating first visit (baseline) data from follow-up visits, filtering out duplicated or empty entries and removing completely empty variables, streamlining variable names, and organising the data by type of clinical measurement (e.g. combine all laboratory measurements in one data table), among others. Raw unstructured text fields were checked for personal information, which was subsequently either removed while keeping the text field intact or the information was recoded into a new variable that no longer contained the personal information. 


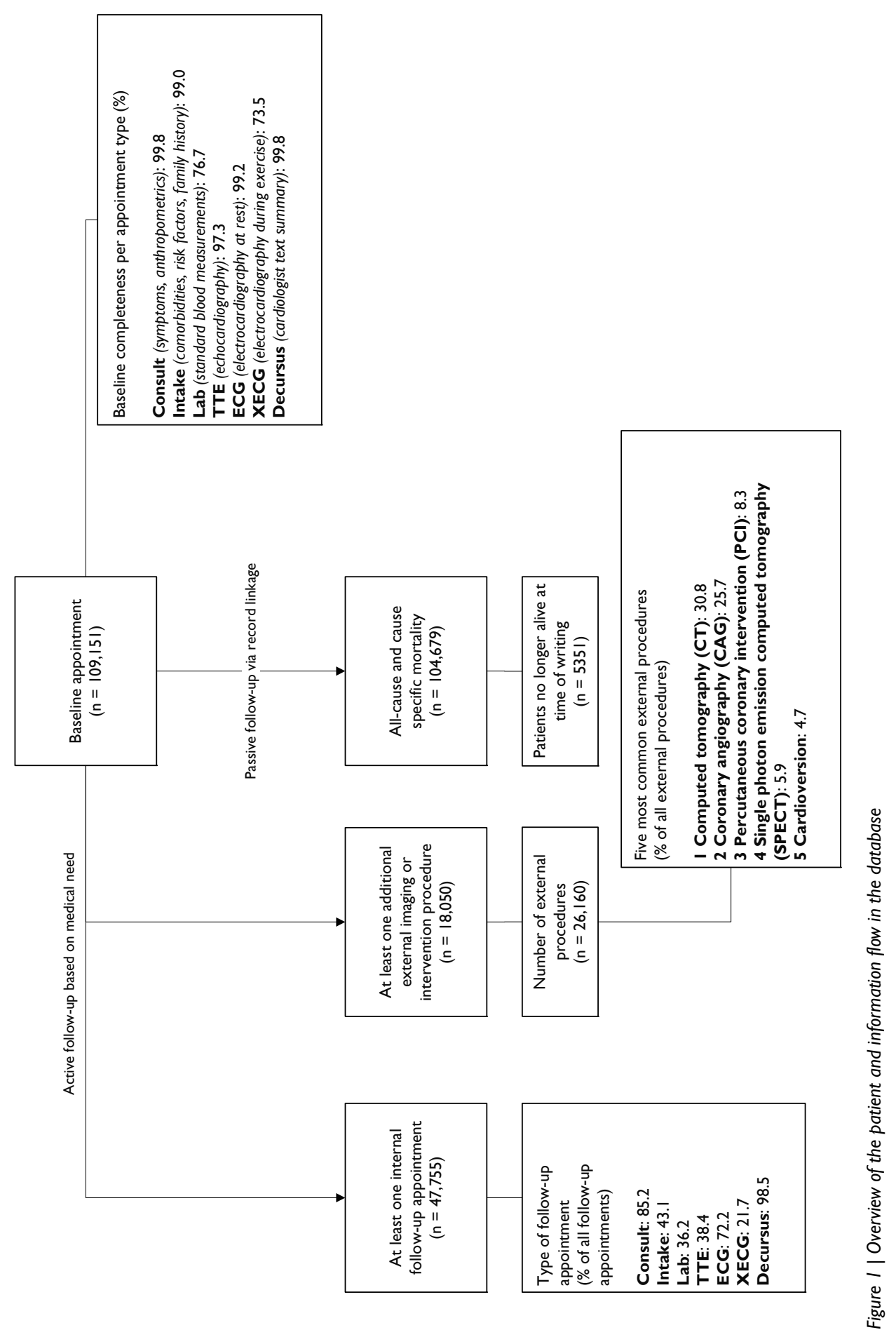


Raw medication use and diagnosis text data were structured into binary variables using text retrieval methods in R ( $\mathrm{R}$ Core Team, Vienna, Austria). Medication entries were grouped into 23 categories of relevant cardiovascular medications based on either the brand name or the generic name, depending on which one was available (Supplementary Table I). Diagnoses were divided into (i) Cardiovascular disease and (ii) Conditions that are risk factors for cardiovascular disease. The first category was subdivided into 5 subgroups, the second one into 4 subgroups (Supplementary Table 2).

The raw data and the clean relational database are stored within the UMCU infrastructure. The raw data is not available for researchers due to privacy constraints and is kept by the data manager.The pseudonymised versions of raw unstructured text fields are available, including raw medication and diagnosis data. Researchers can contact the authors for collaboration and access to the UMCU infrastructure. When the collaboration and the research topic have been agreed upon, external collaborators can get access to both the CCN database and all services and programmes supported by the UMCU.This includes artificial intelligence and advanced statistical programs. All work within the UMCU infrastructure will be stored, including analysis scripts and results. Access to the UMCU infrastructure will be retracted after the project has finished.

\section{Passive and active follow-up outside the clinical trajectory}

The CCN database has been linked to the national database of Statistics Netherlands for passive follow-up for all-cause and cause-specific mortality, and enrichment of the dataset with demographic and socioeconomic data. Linkage was successful for $96 \%$ of the database (Figure I). Failure to link likely occurred because a patient moved between their CCN visit and the moment of linking, as postal code was one of the linking factors. Linking of the CCN database with Statistics Netherlands was deemed appropriate by the ethical committee of Statistics Netherlands as it was in line with the CCN project aims.

Access to the following data was requested and granted: (i) all-cause and cause-specific mortality, (ii) education level and personal income and (iii) Personal Records Database, which among others contains information on country of birth. Access to the Personal Records Database also enables researchers to obtain a matched sample of the general population for comparison with the $\mathrm{CCN}$ population. In the future, the $\mathrm{CCN}$ database will be linked to other registries, such as the national hospitalisation registry, to obtain information on a more diverse set of outcome measures.

Patients could not be contacted for additional baseline questionnaires or active follow-up due to the pseudonymised nature of the database. 
Missing data

Diagnostic procedures, treatments and follow-up of the patients were performed at the discretion of the treating cardiologist and thus driven by medical indication. This results in missing data for both baseline and follow-up visits. For example, more advanced biomarkers such as brain natriuretic peptide or high-sensitivity troponin will only be measured if the cardiologist suspects serious cardiac problems. Similarly, patients without entries in the medication or diagnosis file can be assumed to not use medication or be free of disease. Imputation strategies can be applied to deal with the missing values, but the preferred strategy depends on whether the data is likely to be missing at random or not. Researchers should be aware of the assumptions they make and describe these in their method section.

\section{Patient privacy}

The CCN data were made available under implied consent and transferred to the UMCU under the Dutch Personal Data Protection Act. Patients were assigned a unique patient number that cannot be traced back to an individual without access to the original $\mathrm{CCN}$ data system, which is not available to UMCU researchers. This results in a pseudonymised database. The Medical Research Ethics Committee of the UMCU declared that the Medical Research Involving Human Subjects does not apply to this study. Unstructured text fields containing personal information were anonymised using an anonymization programme ${ }^{14}$ before being included in the final research database.

\section{Content: describing the CCN study population}

The CCN database contains data from 109,227 patients referred to one of the CCN clinics between February 2007 and February 2018. Patients with missing data on age or sex or without records of their CCN visit were excluded $(n=76)$, bringing the total to 109,15 I individuals with a mean age of 56 ( \pm I5) years, of which $52 \%$ were women. About a third of the patients were 65 years or older and $12 \%$ had two or more comorbidities. Patients had a mean body mass index of $27.4( \pm 20) \mathrm{kg} / \mathrm{m}^{2}$ and an average systolic blood pressure of I4 I $( \pm 22) \mathrm{mmHg}$. The majority of patients had a positive cardiovascular family history $(65 \%)$ and I5\% of patients suffered from cardiovascular disease at baseline. Approximately one third of patients were current smokers (37\%), $30 \%$ had hypertension, $16 \%$ had dyslipidaemia and $8 \%$ had diabetes mellitus (DM) (Table 2).

The majority of patients $(56 \%, n=6 I, 232)$ only had a baseline visit, $18 \%(n=19,1 \mathrm{II})$ had one follow-up visit at CCN, and $26 \%(n=28,808)$ had two or more follow-up visits at $C C N$. Compared with patients who were seen once, those with at least one clinical follow-up appointment were older at baseline (60 vs 54 years), had a higher systolic blood pressure ( 145 vs $138 \mathrm{mmHg}$ ) and were more often current smokers (4I vs 33\%). In addition, they more often had a history of cardiovascular disease (2I vs I I\%), prevalent cardiovascular risk conditions (30 vs 16\%), and comorbidities (Supplementary Table 3). 
In total, I8,050 (I7\%) patients were referred for an external procedure (Figure I). Compared with patients who were not referred, patients with at least one external procedure were older at baseline (60 vs 56 years) and had a higher prevalence of comorbidities and CVD history ( $21 \%$ vs $14 \%)$. Women were referred for an external procedure less often $(46 \%$ vs 53\%) (Supplementary Table 4).

The CCN database consists of data derived from medical care and thus participants were not actively recruited, nor were there explicit in- and exclusion criteria. Data on patients who were not referred to $\mathrm{CCN}$ were not available, so we were unable to compare patients referred to $\mathrm{CCN}$ with those who were not. However, to approximate this comparison, we compared the socioeconomic characteristics of the CCN database to an age- and sexmatched sample of the general population. Patients referred to $\mathrm{CCN}$ were more often of Dutch descent $(77 \%$ vs $71 \%)$ and had a higher median annual personal income (€27,9|4 vs $€ 22,270$ ) than the general population (Table 3).

\section{Utility and discussion}

\section{Utility: intended use and database benefits}

The main strength of the $\mathrm{CCN}$ database lies in its combination of a large study population and a large number of different, and sometimes longitudinal, measurements per individual. Such data is difficult to obtain in cohorts specifically set up for research as funds are often not sufficient to cover both including a large population and collecting a large number of (longitudinal) measurements. In addition, the CCN database captures a unique population situated between the GP and the hospital that is rarely seen in clinical studies.

Clinical care databases like the $\mathrm{CCN}$ database can make important contributions to three areas of research due to some of their inherent characteristics. First, these databases reflect the population currently seen in clinical care and thus include groups that are traditionally underrepresented in research ${ }^{8}$. We show that women comprise $52 \%$ of the CCN database, providing a valuable foundation for research into both differences between the sexes and women-specific cardiovascular disease presentations and risk factors ${ }^{15,16}$. Similarly, the CCN dataset contains II,78I patients aged 75 years and older and 13,383 patients with two or more comorbidities, offering researchers an opportunity to verify if study outcomes also apply to these patient groups. These numbers illustrate the potential value of the CCN database for addressing research questions about underrepresented patient groups that have remained unanswered due to scarcity of data.

Second, the size of clinical care databases that combine a large study population with a large number of measurements per individual creates opportunities for the application of artificial intelligence methods. The CCN database contains more than 300 informative features on over 100,000 patients that can be used for the development of artificial intelligence-based 
Table 2 | Baseline characteristics of patients in the CCN database

\begin{tabular}{|c|c|c|c|c|}
\hline Variable & $\begin{array}{l}\text { Whole database } \\
n=|09,15|\end{array}$ & $\begin{array}{c}\text { Women } \\
n=56,628\end{array}$ & $\begin{array}{c}\text { Men } \\
n=52,524\end{array}$ & $\begin{array}{c}\text { Missing data } \\
\text { (\%) }\end{array}$ \\
\hline \multicolumn{5}{|l|}{ General } \\
\hline Women (n, \%) & $56,628(51.9)$ & & & \\
\hline Age (years) & $56(15)$ & $57(15)$ & $56(15)$ & \\
\hline \multicolumn{5}{|l|}{ Age categories (n, \%) } \\
\hline under 50 & $33,165(30.4)$ & 16,954 (29.9) & $|6,2| 1(30.9)$ & \\
\hline $50-64$ & $4 \mathrm{I}, 273(37.8)$ & $20,859(36.8)$ & $20,4 \mid 4$ (38.9) & \\
\hline $65-74$ & $22,931(21.0)$ & $12,152(2 \mid .5)$ & $10,779(20.5)$ & \\
\hline 75 and older & II,78I (I0.8) & $6,662(11.8)$ & $5,119(9.7)$ & \\
\hline Body mass index $\left(\mathrm{kg} / \mathrm{m}^{2}\right)$ & $27.4(20.0)$ & $27.3(20.2)$ & $27.5(19.8)$ & 2.9 \\
\hline Systolic blood pressure $(\mathrm{mmHg})$ & $|4|(22)$ & $140(23)$ & $143(20)$ & 2.9 \\
\hline Current smoker (n, \%) & $40,139(36.8)$ & $20,7 / 2(36.6)$ & 19,427 (37) & 8.9 \\
\hline Ever smoker (n, \%) & $71,659(65.7)$ & $35,508(62.7)$ & $36,151(68.8)$ & 8.8 \\
\hline \multicolumn{5}{|c|}{ Cardiovascular disease (CVD) (n, \%) } \\
\hline History of CVD' & $16,311(14.9)$ & 6,483 (II.4) & $9,828(18.7)$ & \\
\hline Family history of CVD² & $7|| ,48(65.2)$ & $39,318(69.4)$ & $31,830(60.6)$ & 17.8 \\
\hline $\begin{array}{l}\text { History of other cardiovascular } \\
\text { conditions }^{3}\end{array}$ & 23,957 (21.9) & II,804 (20.8) & $12,153(23.1)$ & \\
\hline \multicolumn{5}{|l|}{ Comorbidities (n, \%) } \\
\hline Hypertension & $32,460(29.7)$ & $17,290(30.5)$ & $15,270(28.9)$ & 2.5 \\
\hline Dyslipidaemia & $16,978(15.6)$ & $8,148(14.4)$ & $8,830(16.8)$ & 2.5 \\
\hline Diabetes mellitus & $8,709(8.0)$ & $3,967(7.0)$ & $4,742(9.0)$ & 2.6 \\
\hline \multicolumn{5}{|l|}{ Number of comorbidities } \\
\hline 0 & $64,199(58.8)$ & 33,799 (59.9) & 30,400 (57.9) & \\
\hline I & $28,705(26.3)$ & $|5,08|(26.6)$ & 13,624 (25.9) & \\
\hline 2 & $11,00 \mid(10.1)$ & $5,392(9.5)$ & $5,609(10.7)$ & \\
\hline 3 & $2,382(2.2)$ & $\mathrm{I}, 125(2.0)$ & $\mathrm{I}, 257(2.4)$ & \\
\hline
\end{tabular}

All values are given as mean (SD) unless otherwise specified. 'History of CVD = diagnosis of heart failure, coronary heart disease, cerebrovascular disease or congenital heart disease before baseline appointment, or invasive cardiac intervention. ${ }^{2}$ Family history of CVD = family history of atherosclerosis, sudden death, cardiomyopathy or arrhythmia. ${ }^{3}$ History of other cardiovascular conditions = diagnosis of arrhythmia, valvular disease, cardiomyopathy, atherosclerosis, peripheral artery disease or abdominal aneurysm before baseline appointment, or non-invasive cardiac or peripheral intervention

prediction algorithms and decision support tools. In addition, the CCN database contains several anonymised Dutch free text fields, which can be used for the development of text analysis algorithms specific for Dutch clinical notes. This is an important area of research, as many existing text analysis resources are based on English clinical text ${ }^{17}$. These programmes can subsequently be used to extract and structure valuable information from free text and turn it into a usable format for researchers. 
Table 3 | Sociodemographic characteristics of the CCN database and a sample of the general population matched on year of birth and sex

\begin{tabular}{|c|c|c|}
\hline Variable & $\begin{array}{l}\text { CCN database } \\
(n=104,519)^{*}\end{array}$ & $\begin{array}{c}\text { General population } \\
(n=|04,5| 9)\end{array}$ \\
\hline \multicolumn{3}{|l|}{ Origin $*$ (n, \%) } \\
\hline Native Dutch & $80,692(77.2)$ & $74,042(70.8)$ \\
\hline First generation immigrant & $|5,73|(|5|)$. & $24,592(23.5)$ \\
\hline Second generation immigrant & $8096(7.7)$ & $5884(5.6)$ \\
\hline Annual personal income $(€)$ & $27,9 \mid 4[\mid 4,822-47,344]$ & $22,270[11,900-38,758]$ \\
\hline \multicolumn{3}{|c|}{ Annual personal income groups (n, \%) } \\
\hline Negative or zero & $4760(4.6)$ & $570 \mathrm{I}(5.5)$ \\
\hline$<€ 20.000$ & $33,209(31.8)$ & $33,048(31.6)$ \\
\hline$€ 20.000-€ 50.000$ & $42,325(40.5)$ & $33,906(32.4)$ \\
\hline$€ 50.000-€ 100.000$ & $18,204(17.4)$ & $10,530(10.1)$ \\
\hline$€ 100.000-€ 200.000$ & $4197(4.0)$ & $1675(1.6)$ \\
\hline$\geq € 200.000$ & $1121(1.1)$ & $337(0.3)$ \\
\hline Not available & $703(0.7)$ & $19,321(18.5)$ \\
\hline
\end{tabular}

Values are given as median (IQR) unless otherwise specified. * Year of birth could not be re-calculated for 160 study participants, so these could not be matched with the general population and are thus removed from this table. ${ }^{*}$ Origin was defined as (i) Native Dutch; both parents born in the Netherlands, (ii) First generation immigrant; person born outside the Netherlands with at least one parent born outside the Netherlands, (iii) Second generation immigrant; person born in the Netherlands with at least one parent born outside the Netherlands.

Third, clinical care databases reflect medical practice allowing for comparisons between clinical care and the recommendations in the prevailing guidelines. Such perspectives spark debate on inconsistencies that may exist between guidelines and current practice. The CCN database functions in this case as a tool to bridge the gap between guidelines based mainly on clinical research and the reality of daily cardiac care.

\section{Discussion: compare performance and functionality with similar existing databases}

However, the CCN database also has some limitations that need to be addressed. We will discuss the two main ones, data quality and generalisability.

Data quality: missing data and measurement errors

The data within the $\mathrm{CCN}$ database was collected for care purposes and not for research.As a result, data collection and follow-up during the medical trajectory are not uniform across patients. Similarly, the database may not contain all clinical information researchers need, such as highly specific biomarkers, because these are not normally collected in daily care. Furthermore, raw ECG data and echocardiographic images were saved to a different system than the standardised clinical data and were thus not stored in the CCN database. These limitations are in part inherent to the database, so researchers should consider whether the CCN database is 'fit for purpose' for their specific research question. However, some 
of these limitations can be addressed and alleviated. To obtain standardised follow-up for all individuals in the CCN database, we performed record linkage for all-cause and causespecific mortality. We plan to include follow-up for non-fatal outcomes in the future, as these outcomes are clinically relevant for the relatively young and healthy $\mathrm{CCN}$ population. To alleviate the issue of missing data on important confounders such as socioeconomic status, we enriched the CCN database with information on ethnicity, education level and personal income through record linkage. Text mining approaches can be used to further enrich the $\mathrm{CCN}$ database if the required information can be found within the unstructured text fields. Available missing data techniques such as multiple imputation can be used to address remaining missing values as long as researchers carefully consider the assumptions underlying these techniques.

Data collection and entry in the CCN database is not checked as vigorously as in databases created for research, so data entry mistakes and slightly differential measurement practices across CCN clinics may introduce measurement error and misclassification. We have tried to correct the most obvious data entry errors to reduce their effect, but researchers should consider the possibility of differential measurement error and the resulting risk of misclassification bias when interpreting their results.

\section{Generalisability and comparison to other databases}

The CCN database is comprised of patients who were referred by their GP on suspicion of cardiac disease. We were unable to compare those included in the CCN database with those who were not referred, but we were able to approach this comparison by using an age- and sex-matched sample from the general population. We show that CCN patients have a higher socioeconomic status and are more often native Dutch compared with the general population. Moreover, the prevalence of DM in the CCN database seems to be similar to that in the Netherlands as a whole ${ }^{18}$, while we expected a higher prevalence given that CCN screens patients at elevated cardiovascular disease risk. However, GPs may refer DM patients with cardiac complaints to a DM-specific outpatient clinic instead of a CCN clinic, resulting in a low DM prevalence within the $\mathrm{CCN}$ database. This suggests there is some selection bias occurring within the clinical care pathway, where relatively healthy Dutch patients with higher socioeconomic status are more often referred to a $\mathrm{CCN}$ clinic than those with lower socioeconomic status or those of non-Dutch descent.

There are examples of other clinical care databases such as the hospital-based UPOD database $^{19}$ and the Julius General Practitioner's Network ${ }^{20}$. However, these include distinctively different patient populations, as the first collects data from within the hospital and the second from within GP practice. The CCN database is unique in that it captures the patients in between these two. 


\section{Conclusion}

The $\mathrm{CCN}$ database is a regular care database containing data from 109, 15 I patients collected between 2007 and 2018. This database offers the opportunity to perform research in a unique study population that reflects the patient population seen in daily cardiology practice, including women, the elderly, and patients with multiple comorbidities. The size of this database facilitates the application of artificial intelligence methods. Moreover, the features in the database make it possible to describe current cardiology practice and evaluate this against guidelines based primarily on results from clinical trials.

\section{Acknowledgements}

The authors want to thank Aisha Gohar, Enja Blasse, Jonne Emanuel-Hos, and Gideon Valstar for their contributions to data extraction and data management.

\section{Funding}

This study was funded by the Dutch Heart Foundation (2018B017, CVON-Al) and by ZonMw (849100003, Reviews en Kennissyntheses Gender en Gezondheid). The funding instanced had no role in study design, data collection, decision to publish or preparation of the manuscript.

\section{Competing interests}

FA is supported by UCL Hospitals NIHR Biomedical Research Centre. GS, IT and LH are employed by Cardiology Centers of the Netherlands. The other authors have no conflict of interest to declare. 


\section{References}

I. Roth GA, Abate $\mathrm{D}$, Abate $\mathrm{KH}$, et al. Global, regional, and national age-sex-specific mortality for 282 causes of death in 195 countries and territories, 1980-2017: a systematic analysis or the Global Burden of Disease Study 20I7.The Lancet 2018; 392(10I59): 1736-88.

2. Vos T, Abajobir AA, Abate $\mathrm{KH}$, et al. Global, regional, and national incidence, prevalence, and years lived with disability for 328 diseases and injuries for 195 countries, 1990-2016: a systematic analysis for the Global Burden of Disease Study 2016. The Lancet 2017; 390(10100): I21 I-59.

3. ten Berg MJ, Huisman A, van den Bemt PM, Schobben AF, Egberts AC, van Solinge WW. Linking laboratory and medication data: new opportunities for pharmacoepidemiological research. Clinical chemistry and laboratory medicine 2007; 45(I): I3-9.

4. Johnston SS, Morton JM, Kalsekar I, Ammann EM, Hsiao C-W, Reps J. Using Machine Learning Applied to RealWorld Healthcare Data for Predictive Analytics:An Applied Example in Bariatric Surgery.Value in Health 2019; 22(5): 580-6.

5. Rajkomar A, Oren E, Chen K, et al. Scalable and accurate deep learning with electronic health records. NPJ Digital Medicine 20I8; I(I): I8.

6. Samad MD, Ulloa A, Wehner GJ, et al. Predicting survival from large echocardiography and electronic health record datasets: optimization with machine learning. JACC: Cardiovascular Imaging 2018: 2641.

7. Pilote L, RaparelliV. Participation ofWomen in Clinical Trials: Not Yet Time to Rest on Our Laurels. Journal of the American College of Cardiology 2018; 7I(I8): 1970-2.

8. Sardar MR, Badri M, Prince CT, Seltzer J, Kowey PR. Underrepresentation of Women, Elderly Patients, and Racial Minorities in the Randomized Trials Used for Cardiovascular GuidelinesUnderrepresentation of in Randomized Trials Letters. JAMA Internal Medicine 20 I4; I74(I I): I868-70.

9. Van Spall HGC,Toren A, Kiss A, Fowler RA. Eligibility Criteria of Randomized Controlled Trials Published in HighImpact General Medical JournalsA Systematic Sampling Review. JAMA 2007; 297( I I): I 233-40.

10. Garcia M, Mulvagh SL, Merz CNB, Buring JE, Manson JE. Cardiovascular Disease in Women. Circ Res 20 I 6; I I 8(8): 1273-93.

II. Jagannathan R, Patel SA,Ali MK, Narayan KMV. Global Updates on Cardiovascular Disease Mortality Trends and Attribution of Traditional Risk Factors. Current diabetes reports 2019; 19(7): 44.

12. Seidell JC, Halberstadt J. The global burden of obesity and the challenges of prevention. Annals of nutrition \& metabolism 2015; 66 Suppl 2: 7-12.

13. Gentile BA. Contraindications to Stress Testing. In:A TD,A GB, eds. Pocket Guide to Stress Testing; 20I9: 45-52.

14. Menger V, Scheepers F, van Wijk LM, Spruit M. DEDUCE: A pattern matching method for automatic deidentification of Dutch medical text. Telematics and Informatics 20I8; 35(4): 727-36.

15. Groepenhoff F, Eikendal ALM, Onland-Moret NC, et al. Coronary artery disease prediction in women and men using chest pain characteristics and risk factors: an observational study in outpatient clinics. BMJ Open 2020; I0(4): e035928.

16. Siegersma KR, Groepenhoff F, Onland-Moret NC, et al. New York Heart Association class is strongly associated with mortality beyond heart failure in symptomatic women. Eur Heart J Qual Care Clin Outcomes 2021; 7(2): 2I4-5.

17. Névéol A, Dalianis H,Velupillai S, Savova G, Zweigenbaum P. Clinical Natural Language Processing in languages other than English: opportunities and challenges. Journal of Biomedical Semantics 2018;9(I): 12.

18. Volksgezondheidenzorg.info. Prevalentie diabetes in huisartsenpraktijk naar leeftijd en geslacht. 2020.https:// www.volksgezondheidenzorg.info/onderwerp/diabetes-mellitus/cijfers-context/huidige-situatie\#nodeprevalentie-diabetes-huisartsenpraktijk-naar-leeftijd-en-geslacht2020).

19. Berg MJt, Huisman A, Bemt PMLAvd, Schobben AFAM, Egberts ACG, Solinge WWv. Linking laboratory and medication data: new opportunities for pharmacoepidemiological research. Clinical Chemistry and Laboratory Medicine (CCLM) 2007; 45(I): I3.]

20. Smeets HM, Kortekaas MF, Rutten FH, et al. Routine primary care data for scientific research, quality of care programs and educational purposes: the Julius General Practitioners' Network (JGPN). BMC Health Services Research 2018; 18(I): 735. 


\section{Supplementary files}

\section{Supplementary tables}

Supplementary Table I | Medication names per medication group

\begin{tabular}{|c|c|}
\hline Group & Medications included \\
\hline Aspirin & Acetylsalicic acid, carbasalate calcium \\
\hline $\begin{array}{l}\text { Angiotensin-converting } \\
\text { enzyme inhibitors }\end{array}$ & $\begin{array}{l}\text { Benazepril, perindopril, captopril, cilazapril, delapril, enalapril, fosinopril, lisinopril, } \\
\text { quinapril, ramipril, trandolapril, zofenopril }\end{array}$ \\
\hline $\begin{array}{l}\text { Angiotensin II receptor } \\
\text { blockers }\end{array}$ & Candesartan, eprosartan, irbesartan, losartan, olmesartan, telmisartan, talsartan \\
\hline Thiazides & Hydrochlorothiazide, chlorthalidone, indapamide \\
\hline Potassium-sparing diuretics & Eplerenone, spironolactone, triamterene \\
\hline Loop diuretics & Bumetanide, furosemide \\
\hline Beta-blockers & $\begin{array}{l}\text { Acebutolol, atenolol, bisoprolol, carvedilol, celiprolol, labetalol, metoprolol, } \\
\text { nebivolol, pindolol, propranolol, sotalol }\end{array}$ \\
\hline Calcium-channel blockers & $\begin{array}{l}\text { Amlodipine, barnidipine, felodipine, isradipine, , lacidipine, lercanidipine, } \\
\text { nicardipine, nifedipine, nimodipine, nitrendipine, diltiazem, verapamil }\end{array}$ \\
\hline Alpha-blockers & Alfuzosine, doxazosine, silodosine, tamsulosine, terazosine, urapidil \\
\hline Nitrates & Isosorbide dinitrate, , isosorbide mononitrate, nicorandil, nitroglycerine \\
\hline Digoxin & Digoxin \\
\hline Statins & Atorvastatine, fluvastatine, pitavastatine, pravastatine, rosuvastatine, simvastatine \\
\hline Metformin & Metformin \\
\hline Insulin & Insuline \\
\hline Ezetimibe & Ezetimibe \\
\hline Sulphonylureas & Glibenclamide, glimepiride, tolbutamide, gliclazide \\
\hline Fibrates & Bezafibrate, ciprofibrate, gemfibrozil, fenofibrate \\
\hline P2Y 12 inhibitors & Prasugrel, clopidogrel, ticagrelor \\
\hline Dipyridamole & Dipyridamole \\
\hline Ivabradine & Ivabradine \\
\hline $\begin{array}{l}\text { Non-vitamin } \mathrm{K} \text { oral } \\
\text { anticoagulants }\end{array}$ & Apixaban, edoxaban, rivaroxaban, dabigatran \\
\hline Anti-arrhythmics & $\begin{array}{l}\text { Amiodarone, disopyramide, flecainide, kinidine, lidocaine } \\
\text { propafenone }\end{array}$ \\
\hline Vitamin $\mathrm{K}$ antagonists & Acenocoumarol, phenprocoumon \\
\hline Other & Any medication that is not in any of the groups described above \\
\hline
\end{tabular}


Supplementary Table 2 | Diagnoses per diagnosis group

\begin{tabular}{|c|c|}
\hline Group & Diagnoses included \\
\hline \multicolumn{2}{|c|}{ Cardiovascular disease } \\
\hline Heart failure & $\begin{array}{l}\text { Left ventricular hypertrophy, left ventricular dysfunction, concentric hypertrophic } \\
\text { left ventricle, concentric left ventricle, decompensatio cordis, heart failure, diastolic } \\
\text { dysfunction, coronary microvascular disease, poor ventricular function }\end{array}$ \\
\hline $\begin{array}{l}\text { Coronary heart } \\
\text { disease }\end{array}$ & $\begin{array}{l}\text { Myocardial infarction, angina pectoris, anginal symptoms, chest pain, acute coronary } \\
\text { syndrome, silent ischaemia, coronary disease, heart revalidation, coronary insufficiency, } \\
\mathrm{I} / 2 / 3 \text { artery disease }\end{array}$ \\
\hline $\begin{array}{l}\text { Cerebrovascular } \\
\text { disease }\end{array}$ & $\begin{array}{l}\text { Cerebrovascular accident, transient ischaemic attack, subarachnoid haemorrhage, eye } \\
\text { infarct, brain infarct, brain bleeding, stroke, subarachnoidal bleeding, cerebral infarct, } \\
\text { cerebrovascular infarct, retina infarct, lacunar infarct }\end{array}$ \\
\hline $\begin{array}{l}\text { Congenital heart } \\
\text { disease }\end{array}$ & $\begin{array}{l}\text { Tetralogy of Fallot, ventricular septum defect, atrial septum defect, septum defect, } \\
\text { coarctatio aortae, foramen ovale, Ductus Botalli }\end{array}$ \\
\hline $\begin{array}{l}\text { Cardiovascular } \\
\text { intervention }\end{array}$ & $\begin{array}{l}\text { Percutaneous coronary intervention, stent, coronary artery bypass graft, bypass, } \\
\text { revascularisation, grafting, dotter, percutaneous transluminal coronary angioplasty, } \\
\text { valve replacement, valvuloplasty, transcatheter aortic valve implantation, aortic valve } \\
\text { replacement, mitral valve replacement, commissurotomy, myocardial perfusion scan, heart } \\
\text { catheterisation, implementation of pacemaker or implantable cardioverter-defibrillator }\end{array}$ \\
\hline \multicolumn{2}{|c|}{ Conditions that are risk factors for cardiovascular disease } \\
\hline $\begin{array}{l}\text { Other cardiovascular } \\
\text { disease }\end{array}$ & $\begin{array}{l}\text { Cardiomyopathy, atherosclerosis, abdominal aortic aneurysm, peripheral vascular disease, } \\
\text { arteriosclerosis, claudicatio intermittens, deep vein thrombosis, venous thrombosis, } \\
\text { venous insufficiency, phlebitis }\end{array}$ \\
\hline Arrhythmia & $\begin{array}{l}\text { Atrial fibrillation, ventricular fibrillation, atrium flutter, ventricular flutter, paroxysmal } \\
\text { atrial fibrillation, conduction delay, supraventricular tachycardia, sick sinus syndrome, } \\
\text { sinus exit block, Wolff-Parkinson-White, atrioventricular nodal re-entry tachycardia, } \\
\text { extrasystoles, ventricular extrasystoles, arrhythmia, bradycardia, tachycardia, bigemini, } \\
\text { AV block, right bundle branch block, left bundle branch block, left anterior hemiblock, } \\
\text { premature ventricular contractions, premature atrial contractions, atrial extrasystoles, } \\
\text { hemiblock, rhythm disorder }\end{array}$ \\
\hline Valvular disease & $\begin{array}{l}\text { Valve stenosis, valve sclerosis, valve insufficiency, regurgitation, mitral insufficiency, } \\
\text { tricuspid insufficiency, valve defect, mitral regurgitation, mitral stenosis, valve disease }\end{array}$ \\
\hline $\begin{array}{l}\text { Risk factor } \\
\text { intervention }\end{array}$ & $\begin{array}{l}\text { Ablation, radiofrequency catheter ablation, cardioversion, electrocardioversion, } \\
\text { percutaneous transluminal angioplasty, endarterectomy, aortic bifurcation prosthesis, } \\
\text { abdominal aortic stent }\end{array}$ \\
\hline
\end{tabular}


Supplementary Table 3 | Baseline characteristics stratified by follow-up status

\begin{tabular}{|c|c|c|c|c|}
\hline Variable & $\begin{array}{l}\text { Whole cohort } \\
n=|09,| 5 \mid\end{array}$ & $\begin{array}{l}\text { Follow-up } \\
n=47,755\end{array}$ & $\begin{array}{c}\text { No follow-up } \\
n=61,396\end{array}$ & $\begin{array}{c}\text { Missing data } \\
(\%)\end{array}$ \\
\hline \multicolumn{5}{|l|}{ General } \\
\hline Women (n, \%) & $56,628(51.9)$ & $24,27 \mid(50.8)$ & $32,357(52.7)$ & \\
\hline Age (years) & $56(15)$ & $60(14)$ & $54(16)$ & \\
\hline Body mass index $\left(\mathrm{kg} / \mathrm{m}^{2}\right)$ & $27.4(20.0)$ & $27.8(24.4)$ & $27.0(15.9)$ & 2.9 \\
\hline Systolic blood pressure $(\mathrm{mmHg})$ & $|4|(22)$ & $145(22)$ & $138(20)$ & 2.9 \\
\hline Current smoker (n, \%) & $40,139(36.8)$ & $19.645(4 I .1)$ & $20,494(33.4)$ & 8.9 \\
\hline Ever smoker (n, \%) & $71,659(65.7)$ & 34,250 (7I.7) & $37,409(60.9)$ & 8.8 \\
\hline \multicolumn{5}{|c|}{ Cardiovascular disease (CVD) (n, \%) } \\
\hline History of CVD & $|6,3| 1(14.9)$ & $9,845(20.6)$ & $6,466(10.5)$ & \\
\hline Family history of CVD & $71, \mid 48(65.2)$ & $31,125(65.2)$ & $40,023(65.2)$ & 17.8 \\
\hline CVD risk factor conditions & $23,957(21.9)$ & $14,465(30.3)$ & $9,492(15.5)$ & \\
\hline \multicolumn{5}{|l|}{ Comorbidities (n, \%) } \\
\hline Hypertension & $32,460(29.7)$ & I7,238 (36.1) & I5,222 (24.8) & 2.5 \\
\hline Dyslipidaemia & $16,978(15.6)$ & $8,765(18.4)$ & $8,213(13.4)$ & 2.5 \\
\hline Diabetes mellitus & $8,709(8.0)$ & $4,329(9.1)$ & $4,380(7.1)$ & 2.6 \\
\hline
\end{tabular}

Supplementary Table 4 | Baseline characteristics stratified by external referral status

\begin{tabular}{|c|c|c|c|c|}
\hline Variable & $\begin{array}{l}\text { Whole cohort } \\
n=|09,15|\end{array}$ & $\begin{array}{l}\text { External } \\
\text { procedure } \\
n=\mid 8,050\end{array}$ & $\begin{array}{c}\text { No external } \\
\text { procedure } \\
n=91,101\end{array}$ & $\begin{array}{c}\text { Missing data } \\
\text { (\%) }\end{array}$ \\
\hline \multicolumn{5}{|l|}{ General } \\
\hline Women (n ,\%) & $56,628(51.9)$ & $8,322(46.1)$ & $48,306(53.0)$ & \\
\hline Age (years) & $56(15)$ & $60(12)$ & $56(16)$ & \\
\hline Body mass index $\left(\mathrm{kg} / \mathrm{m}^{2}\right)$ & $27.4(20.0)$ & $27.7(15.3)$ & $27.3(20.8)$ & 2.9 \\
\hline Systolic blood pressure $(\mathrm{mmHg})$ & $|4|(22)$ & I44 (2I) & $|4|(22)$ & 2.9 \\
\hline Current smoker (n, \%) & $40,139(36.8)$ & $6,169(34.2)$ & $33,970(37.3)$ & 8.9 \\
\hline Ever smoker (n, \%) & 71,659 (65.7) & $|I, 98|(66.4)$ & $59,678(65.5)$ & 8.8 \\
\hline \multicolumn{5}{|c|}{ Cardiovascular disease (CVD) (n, \%) } \\
\hline History of CVD & $|6,31|(14.9)$ & $3,839(21.3)$ & $12,472(13.7)$ & \\
\hline Family history of CVD & $7||$,48 (65.2) & $12,492(69.2)$ & $58,656(64.4)$ & 17.8 \\
\hline CVD risk factor conditions & 23,957 (21.9) & $4,531(25.1)$ & 19,426 (21.3) & \\
\hline \multicolumn{5}{|l|}{ Comorbidities (n, \%) } \\
\hline Hypertension & $32,460(29.7)$ & $6,389(35.4)$ & 26,07I (28.6) & 2.5 \\
\hline Dyslipidaemia & $16,978(15.6)$ & $3,583(19.9)$ & I3,395 (|4.7) & 2.5 \\
\hline Diabetes mellitus & $8,709(8.0)$ & $\mathrm{I}, 864(10.3)$ & $6,845(7.5)$ & 2.6 \\
\hline
\end{tabular}


DESIGN OF THE CCN DATABASE 



\title{
Sex differences in
} the number and type of adverse drug reactions reported for common cardiovascular medications in a cardiac outpatient population

5

\author{
Sophie H. Bots \\ Milena Jancev \\ N. Charlotte Onland-Moret \\ Igor I.Tulevski \\ G.Aernout Somsen \\ Hester M. den Ruijter
}




\begin{abstract}
Background

Women report more adverse drug reactions (ADRs) for cardiovascular medications than men according to pharmacovigilance data, but this may be caused by sex differences in prescription rates. We used a clinical care dataset to evaluate sex differences in the prescription-corrected number and type of ADRs reported for common cardiovascular medications.
\end{abstract}

\title{
Methods
}

The Cardiology Centers of the Netherlands database comprises 56,628 women and 52,524 men referred for cardiac work-up by their general practitioner on suspicion of cardiac disease. We included all cardiovascular medications with over 10,000 prescriptions, which were angiotensin-converting enzyme inhibitors, angiotensin II receptor blockers, aspirin, beta-blockers, calcium channel blockers (CCBs), nitrates, statins, and thiazide diuretics. ADRs were defined as the recorded reasons for discontinuing a prescription and categorised into system organ classes following the MedDRA hierarchy. The number of ADRs was corrected for total number of prescriptions per medication group for each sex. The chi-squared test was used to test whether women reported more ADRs than men. Sex differences in number of ADRs were calculated per system organ class to evaluate differences in ADR types.

\section{Results}

We included 63,898 patients ( $50 \%$ women) who received 212,618 prescriptions ( $48 \%$ from women). Men had more prescriptions than women (3 [2-5] vs $2[1-4], p<0.001)$. The number of prescriptions discontinued because of an ADR was higher in women $(n=3516)$ than men $(n=3260)(p<0.00 I)$. After correction for total number of prescriptions, women had more ADR-related discontinued prescriptions than men for all medication classes. The largest difference was seen for CCBs (565 vs 376 ADRs per 10,000 prescriptions, $p<0.001$ ). Nervous system, immune system, and gastrointestinal system disorders were more often reported by women across medication classes, whereas for men these were cardiac and renal and urinary disorders. In case of an ADR, women stopped their prescription after 65 days [2I-266] compared to 105 days in men [32-37I].

\section{Conclusion}

Women more often discontinue their cardiovascular medication prescription because of ADRs, both in absolute numbers and after correction for total number of prescriptions, and do so earlier than men.Women and men reported different ADRs for the same medication classes. These sex differences need to be taken into account in both prescription and monitoring of cardiovascular medications. 


\section{Introduction}

Literature suggests that women treated with cardiovascular medications have a higher risk of adverse drug reactions (ADRs) compared with men'. Biological differences in drug metabolism (pharmacokinetics) and the effect of sex hormones (pharmacodynamics) may underlie the elevated ADR risk in women ${ }^{1-4}$. Having a good understanding of sex-specific ADR risks is important because ADRs can negatively affect adherence and the subsequent potential benefit of treatment ${ }^{5}$. However, efforts at elucidating sex-specific ADRs based on cardiovascular trial data are hampered by the limited incidence of ADRs and the underrepresentation of women in such trials ${ }^{6}$.

Pharmacovigilance data suggest women experience more ADRs than men across all medication types ${ }^{7,8}$ and hint at sex-specific medication-ADR combinations ${ }^{8-10}$, such as a higher number of ACEl-related cough complaints in women'. However, these studies are limited by their lack of information on sex-specific prescription rates. As a result, it is unclear whether the observed differences truly signal sex differences in ADR risk or are driven by differences in prescription rates between women and men. Such prescription data can be obtained via for example a nationwide apothecary registry, which was done in a Dutch study investigating sex differences in ADR-related hospital admissions" ${ }^{\prime \prime}$ They showed that the risk of hospitalisation due to ADRs is higher in women compared with men even after correcting for nationwide prescription", supporting the observations from pharmacovigilance studies. Another observational study opted to incorporate pharmacovigilance in a hospital setting, which allowed them to characterise patients, prescriptions, and ADRs with a high level of detail $^{\prime 2}$. They also found that women experienced more ADRs than men, and that about half of these were dose-related ${ }^{\prime 2}$. However, both of these examples were unable to evaluate milder ADRs occurring outside of the hospital setting, which are especially relevant for adherence to treatment.

The last approach illustrates that clinical care data could be a valuable alternative source of information as they combine the large size of pharmacovigilance databases with information about prescription rates and dosage. This is especially relevant for outpatient settings where information about ADRs is still scarce ${ }^{13}$. Therefore, we used a clinical care database from I3 Dutch cardiac outpatient clinics to evaluate sex differences in the number and type of ADRs reported for commonly prescribed cardiovascular medications after correction for total number of prescriptions.

\section{Methods}

\section{Study database}

The Cardiology Centers of the Netherlands ( $C C N)$ database contains routine clinical care data from 109, I5I patients that were referred for a full cardiac screening workup by their 
general practitioner on suspicion of cardiac disease between 2007 and 2018. During the first visit, information was collected on anthropometric measurements, cardiovascular risk factors, cardiovascular disease history, comorbidities and medication use by a healthcare professional. Information on medication use was recorded per individual prescription and comprised the medication's brand- and/or generic name, the start- and end date of the prescription, the dose, dosage and frequency, and the reason why a prescription ended. Information on medication use was updated during any follow-up visits that occurred.

There were 499,432 unique prescriptions in the CCN database. For the current study, all cardiovascular medication classes with over 10,000 prescriptions were included. This cutoff was chosen to filter out medication classes with too few prescriptions for meaningful comparisons between sexes and across ADR types. This resulted in the selection of eight medication classes, namely angiotensin-converting enzyme inhibitors (ACEls), angiotensin II receptor blockers (ARBs), aspirin, beta-blockers, calcium channel blockers (CCBs), nitrates, statins, and thiazide diuretics (TZDs). Patients could contribute more than one prescription both in the same and in different medication classes.

Relevant medication prescriptions were identified using pattern matching based on a combination of generic and brand names. One-off prescriptions and prescriptions with data entry errors such as the end date being earlier than the start date were excluded. Prescriptions for sporadic use during complaints or at the patient's discretion were excluded, as were prescriptions lacking information on dose.

\section{Prescription-level characteristics}

All prescriptions with a recorded stop date were checked for the reason to discontinue the medication. When this stop reason was the ADR leading to discontinuation, it was categorised into system organ classes according to the MedDRA hierarchy ${ }^{14}$. Prescriptions with stop reasons that were unlikely to signal an ADR ('dosis change') or that were unclear ('other') and prescriptions without a recorded stop reason were not included in ADRrelated analyses. Prescriptions without recorded stop date were categorised as ongoing. The prescription duration in days was calculated for all prescriptions, using the stop date or the end of follow-up (28 February 2018) for ongoing prescriptions. Prescriptions could contribute more than one ADR.

The daily dose for all prescriptions was calculated based on the recorded dose, frequency and dosage. To compare medication dose across individual medication types and medication classes, daily dose was compared to the defined daily dose (in milligrams) proposed by the World Health Organization ${ }^{15}$. Prescriptions were classified as having a lower, equal or higher dose compared to the defined daily dose. For aspirin, this classification was done based on frequency and dosage only because the daily defined dose for medication types in this class was 'I tablet'. Prescriptions for the nitrate medication nitroglycerin spray $(n=27 / 8)$ could 
not be classified because there is no daily defined dose for this medication type. In addition, daily dose could not be calculated for $16 \%$ of prescriptions because frequency information was not recorded. Dosage for these prescriptions was set to missing.

\section{Patient-level characteristics}

For each unique patient contributing at least one prescription, patient-level information including sex, age, body mass index (BMI), systolic blood pressure, comorbidities, smoking behaviour, number of cardiac disease symptoms, and cardiovascular disease history at time of prescription was extracted. The anthropometric measurements and information on comorbidities, symptoms, and smoking were taken from the screening visit closest to the start date of the prescription within a $3 \mathrm{I}$-day period before and after the start date. If there was no visit within this time period, the measurements were set to missing and comorbidities and smoking were classified as 'unknown' (approximately I5\% of patients). Cardiovascular disease diagnoses were classified as history if they occurred before time of prescription and as concurrent diagnosis if they occurred at the same date as the prescription started. The absence of cardiovascular disease diagnose entries was interpreted as being free from cardiovascular disease. The total number of cardiovascular medication prescriptions per unique patient was calculated. The 10-year cardiovascular disease risk was calculated using the SCORE algorithm ${ }^{16}$, but was unavailable for $45 \%$ of patients due to missing data in one or more of the components.

\section{Statistical analysis}

Continuous values are given as mean (standard error) or median [interquartile range], depending on their distribution. Categorical variables are given as number and percentage.

To correct for potential sex differences in prescription, the sex-specific number of ADRs per 10,000 prescriptions was calculated by dividing the total number of ADRs reports by the total number of prescriptions within each medication class and sex. These prescription-corrected ADR numbers were compared between the sexes to evaluate sex differences in the number of ADRs using Pearson's chi-squared test. The relative difference was calculated by dividing the prescription-corrected ADR number in women by that in men. To compare ADR types between the sexes, the absolute sex difference in the prescription-corrected ADRs for each system organ class group was calculated and plotted in bar graphs. To provide context about the frequency of ADRs, the prescription-corrected ADR numbers per system organ class were labelled according to the Council for International Organizations of Medical Sciences $(\mathrm{ClOMS})$ guidelines, which range from very common $(\geq 1 / 10)$ to very rare $(<1 / 10,000)^{17}$.

All analyses were performed in R (R Core Team, Vienna, Austria). A p-value of $<0.05$ was considered statistically significant. 


\section{Results}

\section{Study population}

Eight cardiovascular medication classes with in total 239,469 prescriptions were eligible for inclusion based on the 10,000-prescription cut-off. Of these prescriptions, 26,826 were excluded due to data quality reasons such as missing doses and data entry errors, leaving 212,618 prescriptions to include in this study (Figure I). These prescriptions came from 31,952 women and 31,946 men of similar age (63 \pm 13 vs $62 \pm 12$ years, respectively) and comorbidity profile (Table I). Women had a lower 10-year cardiovascular disease risk compared with men (2.9 [0.9-7.6] vs 4.7 [2.1-9.5]) and less often had a history of cardiovascular disease (19\% vs $30 \%$ ). Women also had fewer prescriptions per person than men (2 [I-4] vs 3 [2-5], respectively) (Table I).

The 212,618 included prescriptions comprised 6776 ADR-related discontinuations (52\% from women) and 143,284 ongoing prescriptions (46\% from women). The remainder were ADR-unrelated discontinuations (Figure I). Women stopped prescriptions 40 days earlier than men when they experienced an ADR (65 [2I-266] vs 105 [2I-37I] days). About one third of the patients received a lower dose compared to defined daily dose, and about one third received a higher dose, but this was similar between the sexes. ADR-related discontinued prescriptions were more often of lower or higher dose compared with ongoing prescriptions in both women (30\% vs $24 \%$ for lower dose and $33 \%$ vs $27 \%$ for higher dose) and men ( $29 \%$ vs $21 \%$ and $37 \%$ vs $29 \%$, respectively) (Table 2 ).

\section{Sex differences in the number of prescriptions and ADRs}

In absolute numbers, men had more prescriptions for ACEls (59\%), aspirin (57\%), nitrates (55\%), and statins (57\%) and fewer for TZDs (46\%) compared with women. Prescriptions for ARBs, beta-blockers and CCBs were similar between the sexes. The proportion of ongoing versus discontinued prescriptions varied across medication classes, ranging from $1-5 \%$ being discontinued due to an ADR. The differences between men and women were small (Table 3).

After correction for the total number of prescriptions, women reported more ADRs than men for all eight medication classes ranging from II\% more for statins to $50 \%$ more for CCBs. This sex difference was statistically significant for ACEls (404 vs 308 ADRs per 10,000 prescriptions, $p<0.001$ ), ARBs (295 vs $237, p=0.012$ ), beta-blockers (383 vs 322, $p=0.022$ ), CCBs (565 vs 376, $p<0.00 I$ ), and nitrates (372 vs $295, p=0.003$ ) (Table 3 ). 


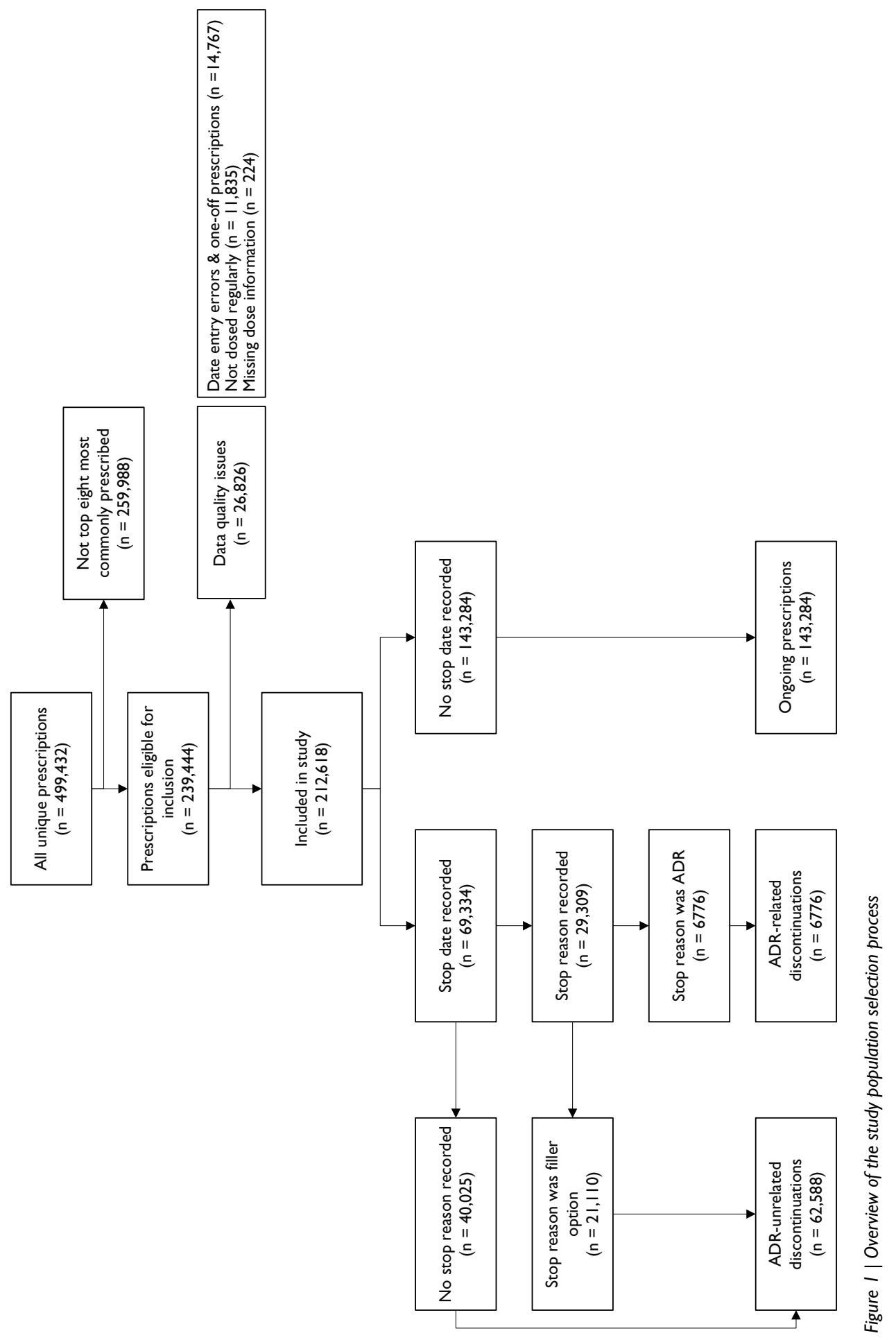


Table I | Patient-level characteristics of all included patients stratified by sex

\begin{tabular}{|c|c|c|}
\hline & $\begin{array}{c}\text { Women } \\
(n=31,952)\end{array}$ & $\begin{array}{c}\text { Men } \\
(n=31,946)\end{array}$ \\
\hline \multicolumn{3}{|l|}{ General characteristics } \\
\hline Age (years) & $63(13)$ & $62(12)$ \\
\hline Body mass index $\left(\mathrm{kg} / \mathrm{m}^{2}\right)$ & $27.1(5.3)$ & $27.4(4.2)$ \\
\hline Systolic blood pressure $(\mathrm{mmHg})$ & $147(23)$ & $\mid 47(2 \mid)$ \\
\hline Current smoker (n, \%) & $10,827(33.9)$ & $10,259(32.1)$ \\
\hline I0-year cardiovascular disease risk (SCORE) & $2.9[0.9-7.6]$ & $4.7[2.1-9.5]$ \\
\hline Cardiovascular medication prescriptions (n) & $2[1-4]$ & $3[2-5]$ \\
\hline \multicolumn{3}{|l|}{ Comorbidities (n, \%) } \\
\hline Hypertension & I5, I $47(47.4)$ & I3,642 (42.7) \\
\hline Dyslipidaemia & $6716(21.0)$ & $7497(23.5)$ \\
\hline Diabetes mellitus & $3383(10.6)$ & $4164(13.0)$ \\
\hline \multicolumn{3}{|c|}{ Cardiovascular disease history and diagnosis (n, \%) } \\
\hline Cardiovascular disease history & $6114(19.1)$ & $9582(30.0)$ \\
\hline Cardiovascular disease diagnosis & $358 \mid(11.2)$ & $4733(14.8)$ \\
\hline Heart failure & $2111(6.6)$ & $265 \mid(8.3)$ \\
\hline Coronary heart disease & $5118(16.0)$ & $8|3|(25.5)$ \\
\hline Cardiovascular intervention & $|7| \mid(5.4)$ & $4955(15.5)$ \\
\hline Cerebrovascular disease & $1923(6.0)$ & $1927(6.0)$ \\
\hline Congenital heart disease & $210(0.7)$ & $162(0.5)$ \\
\hline
\end{tabular}

Sex differences in the type of $A D R s$

General disorders and administration site conditions were reported most often in women $(n=196 \mid)$ and men $(n=1824)$, and they classified as common ADRs after correction for prescription in both sexes (Table 4). The second largest ADR group was surgical and medical procedures ( $n=199$ in women and $n=209$ in men), followed by musculoskeletal and connective tissue disorders ( $n=120$ and $n=\mid 52$, respectively). These groups both classified as uncommon ADRs in both sexes. Nervous system disorders also classified as uncommon ADRs in women, but as rare in men. All other system organ classes were either rare of very rare ADRs in both sexes (Table 4). There was some variation in most often reported ADRs across medication classes, with for example respiratory disorders being the second largest ADR group for ACEls $(n=82)$ and musculoskeletal disorders being the second largest for statins $(n=279)$, but most medication classes followed the general trends described above. However, as the number of reported ADRs in the other medication classes became very small, we could not draw any conclusions on differences in these reported ADRs (Supplementary Figure I). 
Table 2 | Prescription-level characteristics for ongoing and ADR-related discontinued prescriptions stratified by sex

\begin{tabular}{|c|c|c|c|c|}
\hline & \multicolumn{2}{|c|}{ Women } & \multicolumn{2}{|c|}{ Men } \\
\hline & $\begin{array}{c}\text { Ongoing } \\
(n=66,416)\end{array}$ & $\begin{array}{c}\text { Discontinued } \\
\text { due to ADR } \\
(n=35 \mid 6)\end{array}$ & $\begin{array}{c}\text { Ongoing } \\
(n=76,868)\end{array}$ & $\begin{array}{l}\text { Discontinued } \\
\text { due to ADR } \\
(n=3260)\end{array}$ \\
\hline \multicolumn{5}{|l|}{ Medication class (n, \%) } \\
\hline Angiotensin-converting enzyme inhibitors & $6459(9.7)$ & $377(10.7)$ & $9359(12.2)$ & $407(12.5)$ \\
\hline Angiotensin II receptor blockers & 8591 (12.9) & $347(9.9)$ & $8439(1 \mathrm{I} .0)$ & $28 \mid(8.6)$ \\
\hline Aspirin & $9661(14.5)$ & $164(4.7)$ & $13,622(17.7)$ & $183(5.6)$ \\
\hline Beta-blockers & $13,690(20.6)$ & $885(25.2)$ & $13,224(17.2)$ & $757(23.2)$ \\
\hline Calcium channel blockers & $7426($ (I I.2) & $657(18.7)$ & $7744(10.1)$ & $446(13.7)$ \\
\hline Nitrates & $2565(3.9)$ & $162(4.6)$ & $3164(4.1)$ & $156(4.8)$ \\
\hline Statins & $|3,49|(20.3)$ & $777(22.1)$ & $18,432(24.0)$ & $929(28.5)$ \\
\hline Thiazide diuretics & 8585 (12.9) & $308(8.8)$ & $7|4|(9.3)$ & $236(7.2)$ \\
\hline \multicolumn{5}{|l|}{ Prescription characteristics } \\
\hline Prescription duration (days) & $\begin{array}{c}1233 \\
{[454-2|4|]}\end{array}$ & $\begin{array}{c}65 \\
{[2 \mid-266]}\end{array}$ & $\begin{array}{c}1259 \\
{[478-2156]}\end{array}$ & $\begin{array}{c}105 \\
{[32-37 \mid]}\end{array}$ \\
\hline \multicolumn{5}{|l|}{ Number of active compounds ( $n, \%)$} \\
\hline I & $62,470(94.1)$ & $226 I(95.6)$ & $72,775(94.7)$ & $3127(95.9)$ \\
\hline 2 & $3840(5.8)$ & 149 (4.2) & $3929(5.1)$ & $|3|(4.0)$ \\
\hline 3 & $106(0.2)$ & $6(0.2)$ & $164(0.2)$ & $2(0.1)$ \\
\hline \multicolumn{5}{|l|}{ Dosage compared to defined daily dose (n, \%) } \\
\hline Lower & $15,906(23.9)$ & $1055(30.0)$ & I6,403 (2I.3) & $933(28.6)$ \\
\hline Equal & $19,927(30.0)$ & $871(24.8)$ & $24,359(31.7)$ & $744(22.8)$ \\
\hline Higher & $17,696(26.6)$ & I I 68 (33.2) & $22,173(28.8)$ & $1190(36.5)$ \\
\hline Not recorded & $12,887(19.4)$ & $422(12.0)$ & $13,933(18.1)$ & $393(12.1)$ \\
\hline
\end{tabular}

After correction for the total number of prescriptions, women outnumbered men in general disorder and administration site conditions (54 more general ADRs in women per 10,000 prescriptions). This was true for all medication classes, with the largest differences for CCBs ( 152 per 10,000 prescriptions), followed by nitrates (7I per 10,000 prescriptions) and ACEls (66 per 10,000 prescriptions) (Figure 2). For all ADR types, women and men reported a similar number of ADRs after correction for the number of prescriptions. However, some differences were observed between medication classes. Women reported more respiratory ADRs for ACEls (32 more per 10,000 prescriptions), which was mainly driven by coughing complaints. Nervous system disorders such as dizziness and headache, immune system disorders such as allergies, and gastrointestinal disorders such as nausea and stomach pain were in the top five ADR types with more reports in women than men for several medication classes (Figure 2). Interestingly, social disorders were the second largest ADR type with more female reports for statins, which was driven by women stopping prescriptions of their own volition. Cardiac disorders such as arrhythmias and renal and urinary disorders such as kidney dysfunction were in the top five ADR types with more reports in men than women for several medication classes. Interestingly, musculoskeletal and connective tissue disorders such as myalgia were the leading ADR type with more male reports for statins (Figure 2). 


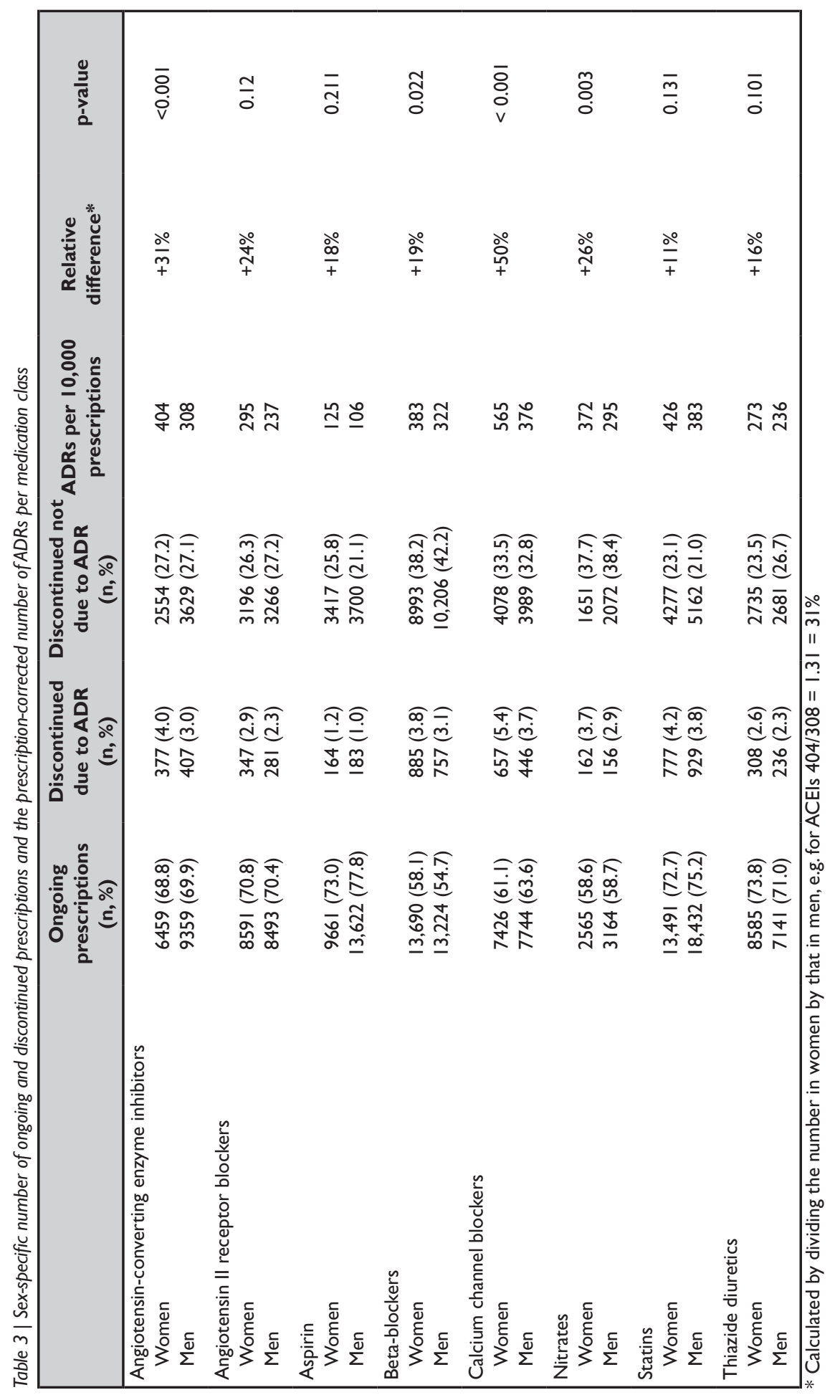




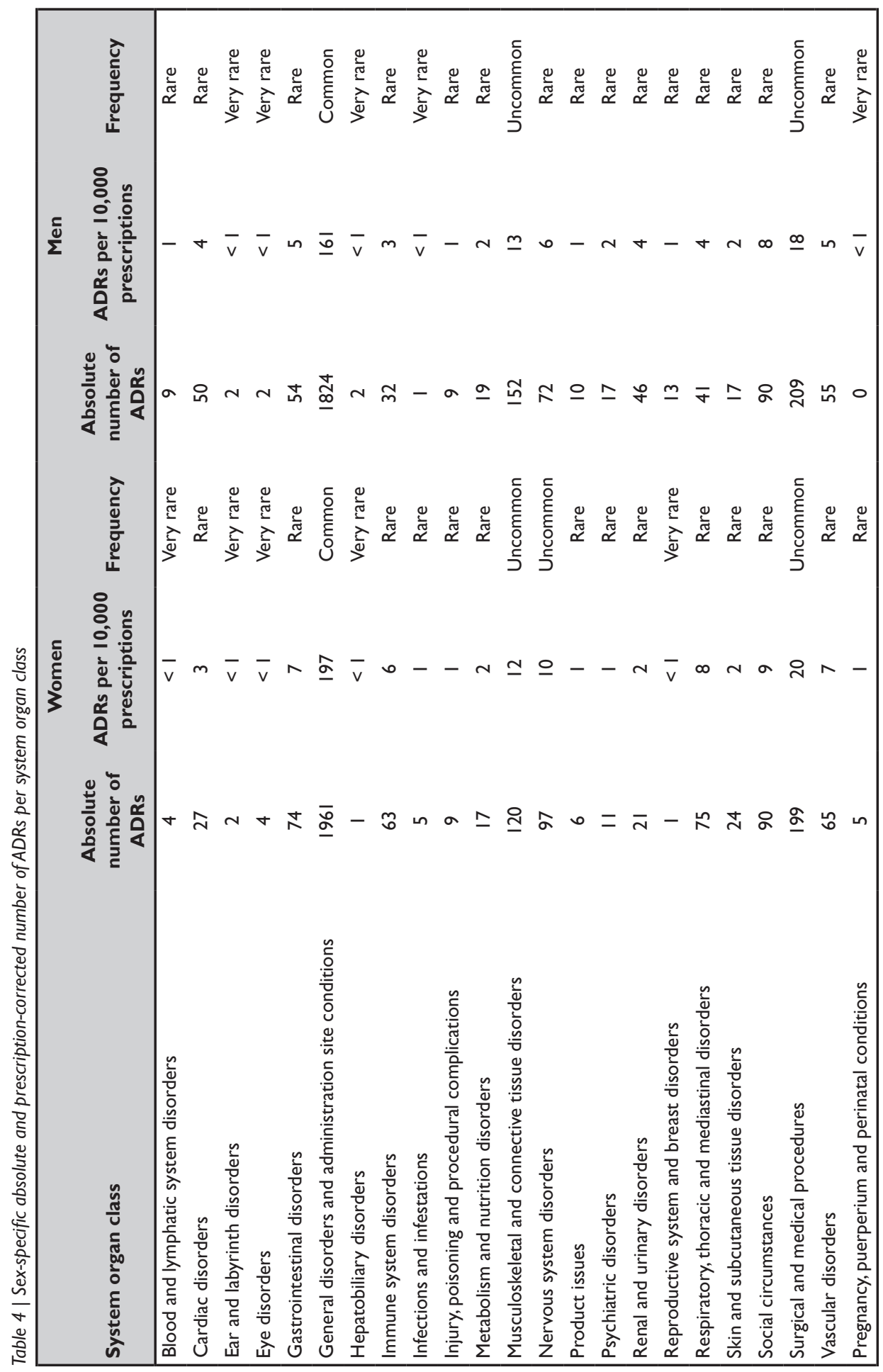




\section{Discussion}

Women seen in outpatient clinics more often discontinue their cardiovascular medication prescription because of an ADR than men, which becomes more pronounced after correction for total number of prescriptions. The largest sex-difference was found for CCBs with a $50 \%$ higher incidence of ADRs in women per 10,000 prescriptions. Women reported more nervous system disorders, respiratory disorders, immune system disorders, and gastrointestinal disorders, whereas men reported more musculoskeletal disorders, renal and urinary disorders, and cardiac disorders. Women also stopped their prescription 40 days earlier than men when they experienced an ADR.

\section{Strengths and limitations}

The strengths of this study are the availability of information on ADRs in combination with total prescription rate and patient-level clinical characteristics, and its size. In addition, as this dataset is a close reflection of daily clinical care in cardiac outpatient clinics, it is a good representation of ADRs commonly encountered in daily clinical practice that result in medication discontinuation. However, this study also has some limitations that need to be addressed. The data collection was not standardised and depended on both patients to truthfully report ADRs and healthcare professionals to correctly enter them. In addition, patients could have stopped their prescription without the CCN cardiologist knowing.

Taken together, this may have led to underreporting of ADRs. Underreporting is inherent to collecting ADR information and also occurs in pharmacovigilance databases ${ }^{18}$, which partially rely on healthcare professionals to relay ADR information to them. As these databases require healthcare professionals to take the additional step of reporting ADRs to them, underreporting in pharmacovigilance databases may be a larger problem compared with our database. This could introduce misclassification, but this would probably affect women and men equally and is thus unlikely to change our conclusions. Possible future solutions include using proxies for ADR-related discontinuations that are independent of clinician or patient reporting behaviour such as medication switches ${ }^{19}$, or employing text retrieval methods to search available free text in the clinical records.

\section{Sex differences in the number of ADR-related discontinuations}

Our finding that women more often stop cardiovascular medication prescriptions due to an ADR supports previous literature reporting higher ADR numbers in women ${ }^{10-12,20,21}$. This sex difference became more pronounced after correction for total number of prescriptions, underlining the importance of prescription information in answering questions about sexspecific ADR-risk. We found that $3 \%$ of prescriptions in our population were discontinued because of an ADR. This prevalence seems low compared to previous work from Sweden, which reported a I-month ADR prevalence of 7.8\% and a 3-month ADR prevalence of 

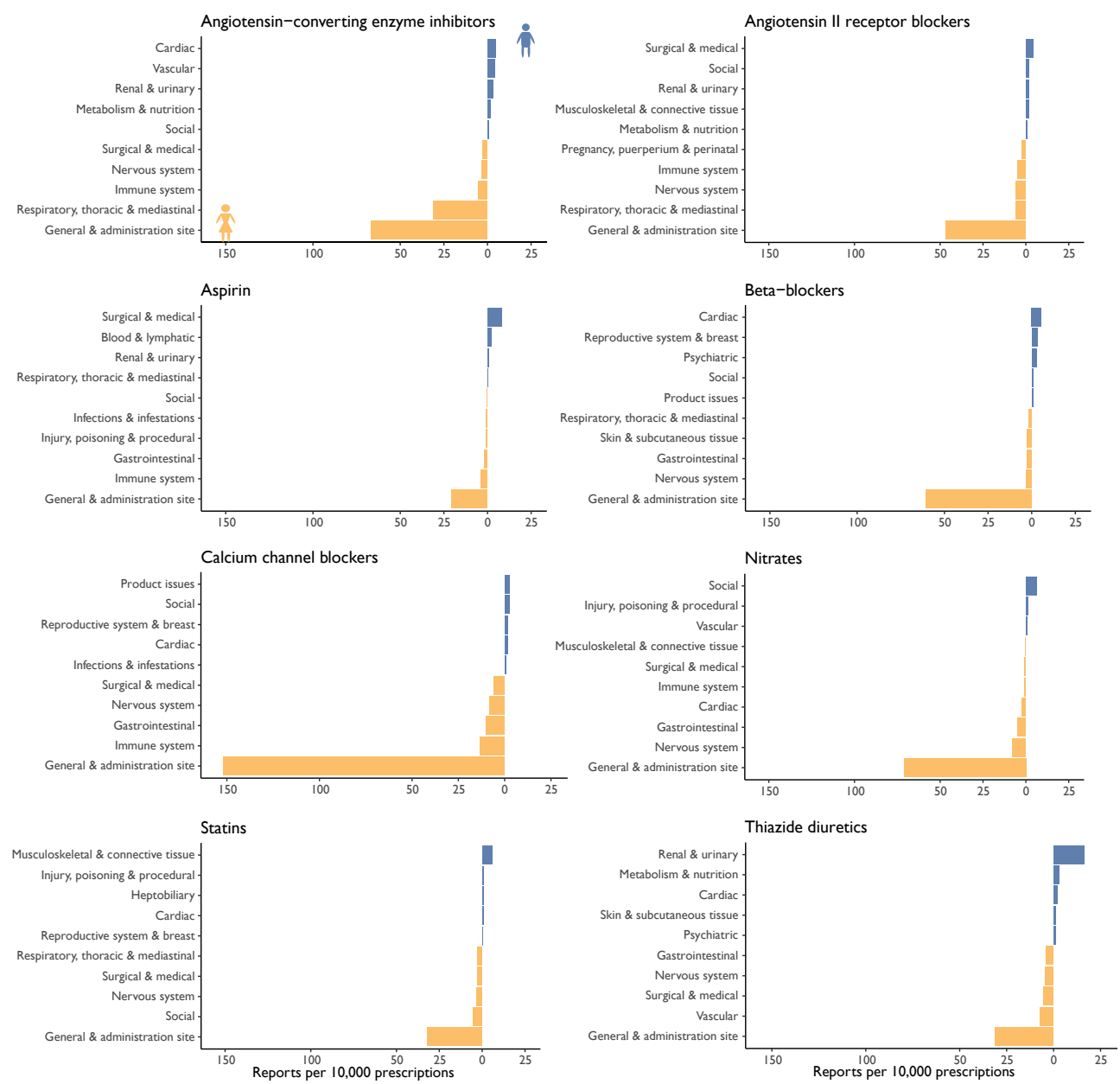

Figure $2 \mid$ Absolute sex difference in number of adverse drug reaction reports for the 10 system organ classes with the largest sex differences stratified by medication class

$6.9 \%$ in the general population 22,23 . A small cohort study from four American primary care practices found that $25 \%$ of patients reported an ADR in the three months after their visit, although they included non-cardiovascular medications as well ${ }^{24}$. However, there are few studies reporting on ADR prevalence in outpatient settings ${ }^{25}$, making it difficult to compare our findings with others. In addition, our study did not include ADRs that did not lead to medication discontinuation, which could explain the lower ADR prevalence.

We showed the largest sex difference for CCBs, where women had 50\% more ADR-related discontinuations per 10,000 prescriptions than men. This is specifically interesting given that $\mathrm{CCBs}$ are one of the recommended medications for the treatment of ischaemia with nonobstructive coronary arteries (INOCA), a coronary artery disease subtype that is present in $50-70 \%$ of women with angina referred for coronary angiography ${ }^{26}$. Given that angina is 
one of the most common symptom of ischaemic heart disease with an estimated global prevalence of 112 million cases ${ }^{26}$, many women worldwide are prescribed CCBs. A similar case can be made for ACEls, which are also recommended for patients with INOCA ${ }^{26}$ but have a $31 \%$ higher incidence of ADR-related discontinuations in women in our dataset. Interestingly, literature from the heart failure field has suggested sex-specific approaches to ACEI therapy out of efficacy considerations, such as sex-specific dosing ${ }^{27}$ or preferentially prescribing $A R B s$ to women instead ${ }^{28}$. The added value of such strategies should be more thoroughly evaluated to find the optimal balance between medication efficacy and safety and test whether this balance is different for women compared with men. Studies such as ours are a first attempt at scoping out medication classes with sex-skewed ADR risk profiles that may benefit most from a sex-specific approach to prescription and dosing.

Sex differences in the type of ADRs reported

Our findings regarding medication class-specific ADR types largely confirm previous work, with a higher prevalence of respiratory ADRs such as cough for $A C E l s^{29}$ and musculoskeletal ADRs such as myalgia for statins ${ }^{30}$.The sex-specific patterns we observed were also similar to previous work, with women reporting more cough for ACEls specifically,31 and more allergic reactions, dizziness, headaches and nausea across cardiovascular medication classes ${ }^{8}$ compared with men. Women also more often stopped their statin prescription on their own volition, which is in line with findings from a large cross-sectional study showing that women in community practice were more likely to refuse or discontinue their statin treatment compared with men ${ }^{32}$. Higher rates of bradycardia and kidney dysfunction in men treated with beta-blockers ${ }^{33}$ or diuretics ${ }^{34}$ have been shown previously, supporting our observation of more cardiac and renal and urinary ADR reports in men compared with women.

We did not see a high prevalence of skin and subcutaneous tissue ADRs such as angiooedema for ACEls, even though angio-oedema is considered an important ACEl-related ADR next to cough ${ }^{35,36}$. Similarly, we found that men reported more myalgia for statins compared to women, even though female sex is considered a risk factor for this $\operatorname{ADR}^{37}$ and women are more aware of muscle-related side effects of statin therapy than men ${ }^{32}$. These findings could be explained by misclassification of angio-oedema and myalgia cases as general oedema or general pain, respectively, which both fall under the general disorders system organ class. These examples illustrate the need for tools that improve the quality of ADR data collected within the clinical care infrastructure. One option is to adopt a systematic approach to ADR classification such as MedDRA in clinical care datasets. Ideally, future clinical care infrastructure incorporates an application that can identify ADRs in written text and link them to potential MedDRA system organ classes in real time, similar to a recently developed pipeline for automatic ICD-I0 code labelling ${ }^{38}$. Such an approach gives the healthcare professional the possibility to pick the best option and adapt their wording if the suggestions that come up are incorrect. This might also result in more specific labelling 
of ADRs, reducing the size of the non-informative general disorders system organ class that now comprised the majority of all ADR reports in our dataset.

To conclude, our findings largely confirm previous work and suggest women report more and different ADRs when treated with common cardiovascular medications compared with men. This information could be used in daily practice to inform patients about their sexspecific ADR risk and aid healthcare professionals in balancing this risk against potential treatment benefit. While better quality data are needed to validate these findings and investigate whether sex-specific optimal dosages reduce ADR risk, studies like these could be a first step in bringing a sex-specific approach to medication prescription to the clinic. 


\section{References}

I. Rosano GM, Lewis B, Agewall S, et al. Gender differences in the effect of cardiovascular drugs: a position document of the Working Group on Pharmacology and Drug Therapy of the ESC. Eur Heart J 20I5; 36(40): 2677-80.

2. Soldin OP, Mattison DR. Sex differences in pharmacokinetics and pharmacodynamics. Clin Pharmacokinet 2009; 48(3): 143-57.

3. Tamargo J, Rosano G, Walther T, et al. Gender differences in the effects of cardiovascular drugs. Eur Heart J Cardiovasc Pharmacother 2017; 3(3): 163-82.

4. Franconi F, Campesi I. Pharmacogenomics, pharmacokinetics and pharmacodynamics: interaction with biological differences between men and women. Br J Pharmacol 20I4; I7I (3): 580-94.

5. Leporini C, De Sarro G, Russo E. Adherence to therapy and adverse drug reactions: is there a link? Expert Opin Drug Saf 2014; 13 Suppl I: S4I-55.

6. Pilote L, Raparelli V. Participation of Women in Clinical Trials: Not Yet Time to Rest on Our Laurels. J Am Coll Cardiol 2018; 7I(18): 1970-2.

7. Watson S, Caster O, Rochon PA, den Ruijter H. Reported adverse drug reactions in women and men: Aggregated evidence from globally collected individual case reports during half a century. EClinicalMedicine 2019; 17: 100188.

8. de Vries ST, Denig P, Ekhart C, et al. Sex differences in adverse drug reactions reported to the National Pharmacovigilance Centre in the Netherlands: An explorative observational study. British Journal of Clinical Pharmacology 2019; 85(7): I507-I5.

9. Alharbi FF, Kholod AAV, Souverein PC, et al. The impact of age and sex on the reporting of cough and angioedema with renin-angiotensin system inhibitors: a case/noncase study in VigiBase. Fundamental \& Clinical Pharmacology 20I7; 3 I(6): 676-84.

10. Yu Y, Chen J, Li D, Wang L,Wang W, Liu H. Systematic Analysis of Adverse Event Reports for Sex Differences in Adverse Drug Events. Sci Rep 2016; 6: 24955.

II. Rodenburg EM, Stricker BH,Visser LE. Sex-related differences in hospital admissions attributed to adverse drug reactions in the Netherlands. Br J Clin Pharmacol 20I I; 7I (I): 95- 104.

12. Zopf Y, Rabe C, Neubert A, et al. Women encounter ADRs more often than do men. Eur J Clin Pharmacol 2008; 64(I0): 999-1004.

13. Khalil H, Huang C. Adverse drug reactions in primary care: a scoping review. BMC Health Serv Res 2020; 20(I): 5-.

14. Activities MDfR. MedDRA Hierarchy / Basics / How to use. 202I. https://www.meddra.org/how-to-use/ basics/hierarchy 202I).

I5. World Health Organization. Defined Daily Dose (DDD). 202I. https://www.who.int/tools/atc-ddd-toolkit/ about-ddd 202I).

16. Conroy RM, Pyörälä K, Fitzgerald AP, et al. Estimation of ten-year risk of fatal cardiovascular disease in Europe: the SCORE project. Eur Heart J 2003; 24(II): 987-I003.

17. Neubert A, Dormann H, Prokosch H-U, et al. E-pharmacovigilance: development and implementation of a computable knowledge base to identify adverse drug reactions. British journal of clinical pharmacology 20I3; 76 Suppl I (Suppl I): 69-77.

18. Hazell L, Shakir SAW. Under-Reporting of Adverse Drug Reactions. Drug Safety 2006; 29(5): 385-96.

19. Mahmoudpour SH,Asselbergs FW, de Keyser CE, et al. Change in prescription pattern as a potential marker for adverse drug reactions of angiotensin converting enzyme inhibitors. International Journal of Clinical Pharmacy 2015; 37(6): 1095-103.

20. McLernon DJ, Bond CM, Hannaford PC, et al. Adverse drug reaction reporting in the UK: a retrospective observational comparison of yellow card reports submitted by patients and healthcare professionals. Drug Saf 2010; 33(9): 775-88.

21. Hofer-Dueckelmann C, Prinz E, Beindl W, et al. Adverse drug reactions (ADRs) associated with hospital admissions - elderly female patients are at highest risk. Int J Clin Pharmacol Ther 201 I; 49(10): 577-86. 
22. Hakkarainen KM, Andersson Sundell K, Petzold M, Hägg S. Prevalence and perceived preventability of selfreported adverse drug events--a population-based survey of 7099 adults. PLoS One 2013; 8(9): e73I66.

23. Hakkarainen KM, Gyllensten H, Jönsson AK, Andersson Sundell K, Petzold M, Hägg S. Prevalence, nature and potential preventability of adverse drug events - a population-based medical record study of 4970 adults. $\mathrm{Br}$ J Clin Pharmacol 2014; 78(I): 170-83.

24. Gandhi TK, Weingart SN, Borus J, et al. Adverse Drug Events in Ambulatory Care. New England Journal of Medicine 2003; 348(I6): I556-64.

25. Bouvy JC, De Bruin ML, Koopmanschap MA. Epidemiology of adverse drug reactions in Europe: a review of recent observational studies. Drug safety 20I5; 38(5): 437-53.

26. Kunadian V, Chieffo A, Camici PG, et al. An EAPCI Expert Consensus Document on Ischaemia with NonObstructive Coronary Arteries in Collaboration with European Society of Cardiology Working Group on Coronary Pathophysiology \& Microcirculation Endorsed by Coronary Vasomotor Disorders International Study Group. European Heart Journal 2020; 4 I (37): 3504-20.

27. Santema BT, Ouwerkerk W, Tromp J, et al. Identifying optimal doses of heart failure medications in men compared with women: a prospective, observational, cohort study. Lancet 2019; 394(I0205): I254-63.

28. Hudson M, Rahme E, Behlouli H, Sheppard R, Pilote L. Sex differences in the effectiveness of angiotensin receptor blockers and angiotensin converting enzyme inhibitors in patients with congestive heart failure--a population study. Eur J Heart Fail 2007; 9(6-7): 602-9.

29. Mahmoudpour SH, Leusink M, van der Putten L, et al. Pharmacogenetics of ACE inhibitor-induced angioedema and cough: a systematic review and meta-analysis. Pharmacogenomics 20I3; 14(3): 249-60.

30. Thompson PD, Panza G, Zaleski A, Taylor B. Statin-Associated Side Effects. J Am Coll Cardiol 20I6; 67(20): 2395-4I0.

31. Goldsmith DJA. Tackling ACE inhibitor cough. The Lancet 1997; 350(9080): 814.

32. Nanna MG, Wang TY, Xiang Q, et al. Sex Differences in the Use of Statins in Community Practice. Circ Cardiovasc Qual Outcomes 2019; 12(8): e005562.

33. Hoppe UC. Beta-blocker induced bradycardia-should we pace? European Journal of Heart Failure 2004; 6(4): 449-5I.

34. Scott J, Jones T, Redaniel MT, May MT, Ben-Shlomo Y, Caskey F. Estimating the risk of acute kidney injury associated with use of diuretics and renin angiotensin aldosterone system inhibitors: $A$ population based cohort study using the clinical practice research datalink. BMC Nephrology 20I 9; 20(I): 48I.

35. Brown T, Gonzalez J, Monteleone C.Angiotensin-converting enzyme inhibitor-induced angioedema: A review of the literature.J Clin Hypertens (Greenwich) 2017; I9(12): 1377-82.

36. Kostis WJ, Shetty M, Chowdhury YS, Kostis JB.ACE Inhibitor-Induced Angioedema: a Review. Curr Hypertens Rep 2018; 20(7): 55.

37. Bitzur R, Cohen H, Kamari Y, Harats D. Intolerance to statins: mechanisms and management. Diabetes Care 2013; 36 Suppl 2(Suppl 2): S325-S30.

38. Sammani A, Bagheri A, van der Heijden PGM, et al. Automatic multilabel detection of ICDI0 codes in Dutch cardiology discharge letters using neural networks. npj Digital Medicine 202I; 4(I): 37. 


\section{Supplementary Figures}
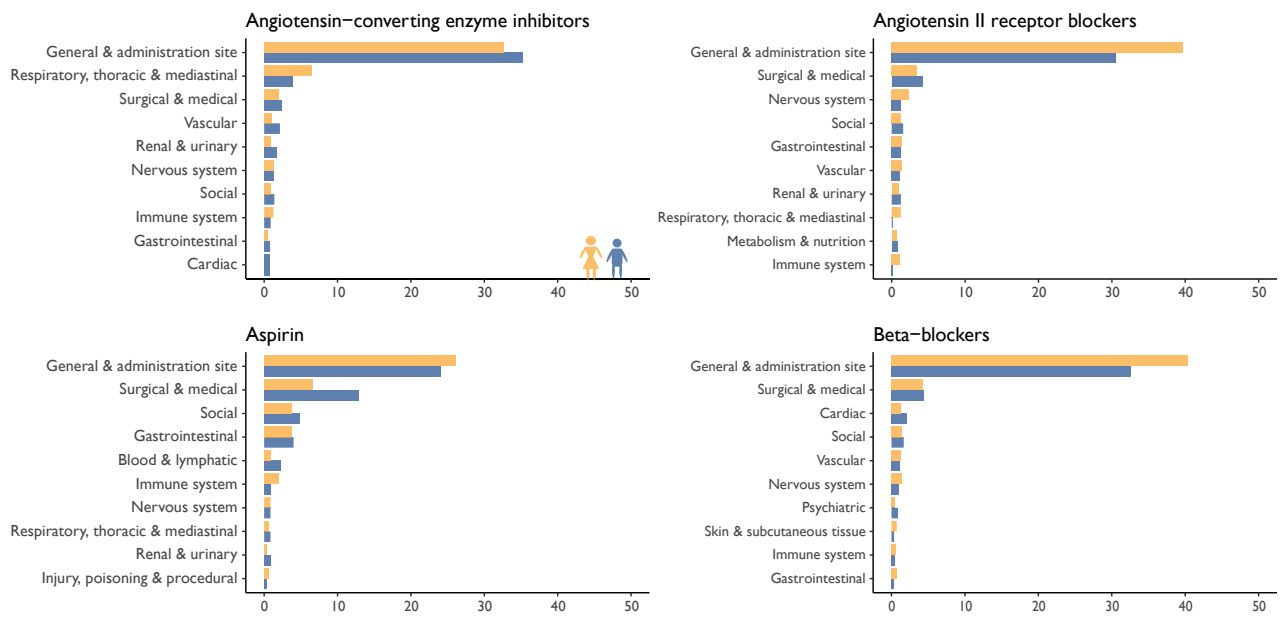

Calcium channel blockers
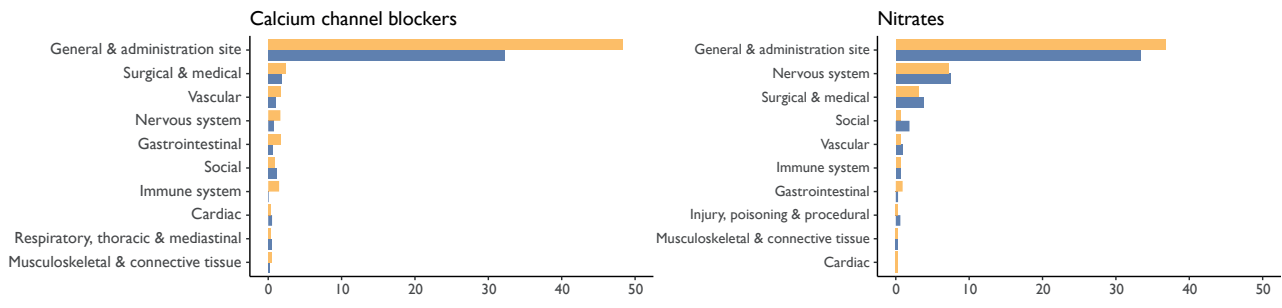

Statins

Thiazide diuretics
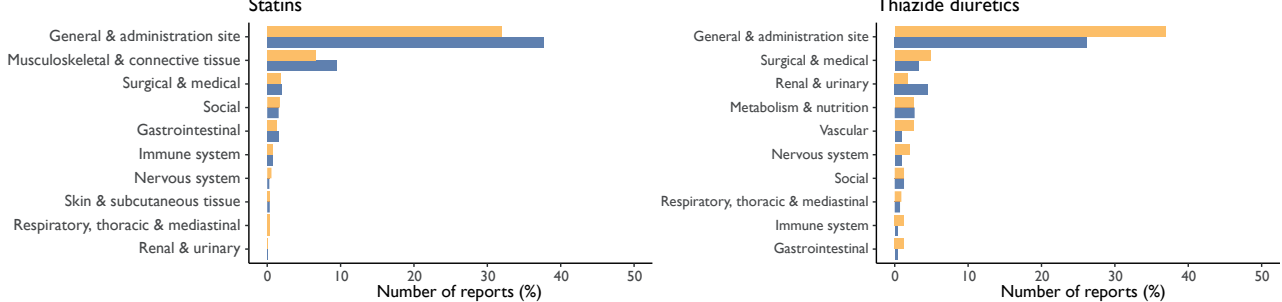

Supplementary Figure I | Sex-specific number of adverse drug reaction reports for the 10 largest system organ classes stratified by medication class 
SEX DIFFERENCES IN ADR NUMBER AND TYPE 



\title{
Heart failure medication dosage and survival in women and men seen at outpatient clinics
}

\author{
6 \\ Sophie H. Bots \\ N. Charlotte Onland-Moret \\ Igor I.Tulevski \\ Pim van der Harst \\ Maarten J. Cramer \\ Folkert W.Asselbergs \\ G.Aernout Somsen \\ Hester M. den Ruijter
}




\begin{abstract}
Objective

Women with heart failure with reduced ejection fraction (HFrEF) may reach optimal treatment effect at half of the guideline-recommended medication dose. This study investigates prescription practice and its relation with survival of heart failure (HF) patients in daily care.

\section{Methods}

Electronic health record data from 13 Dutch outpatient cardiology clinics were extracted for $\mathrm{HF}$ patients receiving at least one guideline-recommended HF medication. Dose changes over consecutive prescriptions were modelled using natural cubic splines. Inverse probabilityweighted Cox regression was used to assess the relationship between dose (reference $\geq$ $50 \%$ target dose) and all-cause mortality.
\end{abstract}

\title{
Results
}

The study population comprised $56 \mathrm{I}$ women ( $29 \% \mathrm{HFrEF}$ (EF<40\%), 49\% HF preserved EF (EF $\geq 50 \%)$; HFpEF) and 615 men (47\% and $25 \%$, respectively). During a median follow-up of 3.7 years, 252 patients died (48\% women; $167 \mathrm{HFrEF}, 84 \mathrm{HFpEF}$ ). Nine hundred thirty-four patients (46\% women) received angiotensin-converting enzyme inhibitors or angiotensin II receptor blockers (ACEI/ARBs), 795 (48\% women) beta-blockers, and 178 (42\% women) mineralocorticoid receptor antagonists (MRAs). In both sexes, the mean target dose across prescriptions was $50 \%$ for ACEI/ARBs and beta-blockers, and 100\% for MRAs. ACEI/ARB dose $<50 \%$ was associated with lower mortality in women but not men with HFrEF. This was not seen in HFpEF patients. Beta-blocker dose was not associated with all-cause mortality.

\section{Conclusions}

HF patients seen in outpatient cardiology clinics receive half of the guideline-recommended medication dose. Lower ACEI/ARB dose was associated with improved survival in women with HFrEF. These results underscore the importance of (re)defining optimal medical therapy for women with HFrEF. 


\section{Introduction}

Heart failure (HF) currently affects 26 million people worldwide', half of which are women². Despite known sex differences in both the presentation of $\mathrm{HF}^{3}$ and the response to drug therapy ${ }^{4}$, HF guidelines recommend the same target doses for women and men 5 . This "one size fits all" approach is debated because women are underrepresented in HF trials and sex-stratified data remains scarce ${ }^{2}$. Efforts to increase the number of women in HF trials have had limited success 6 , possibly because inclusion criteria favour the male pattern of disease. Women with $\mathrm{HF}$ are older, have a preserved ejection fraction $(\mathrm{EF})^{7}$ and lower brain natriuretic peptide levels than men ${ }^{8,9}$. Inclusion criteria based on these characteristics that do not consider inherent sex differences disproportionally exclude women. In addition, HF trials often exclude elderly or multimorbid patients, resulting in study populations healthier than patients seen in daily care ${ }^{10}$. It remains unclear how findings from HF trials translate to real-life patient populations and whether recommendations based on trials are optimal for clinical practice.

Two recent HF with reduced EF (HFrEF) cohorts challenged current recommendations by showing that women receiving $50 \%$ of the guideline-recommended dose for angiotensinconverting enzyme inhibitors (ACEls)/angiotensin-receptor blockers (ARBs) or betablockers had better survival and fewer HF hospitalisations during follow-up than women on higher doses, whereas in men prognosis improved with increasing dose" ${ }^{\text {. Dutch registry }}$ data showed that healthcare professionals often prescribed women with HFrEF lower doses than guideline-recommended, suggesting women already receive lower doses in daily care ${ }^{12}$. Medication dosage in women with HF may additionally be complicated because women more often present with $\mathrm{HF}$ with preserved $\mathrm{EF}(\mathrm{HFpEF})^{7}$, the $\mathrm{HF}$ subtype for which effective treatments and thus information on medication dosage is still lacking. ${ }^{5}$. We used routine care data from Dutch outpatient cardiology clinics to describe current dosage practices in a heterogeneous HF population and investigate whether medication dosage was associated with survival in both sexes and HF subtypes.

\section{Methods}

\section{Study population}

The Cardiology Centers of the Netherlands (CCN) database contains routine clinical care data from 109,15I patients that were referred for cardiac workup by their general practitioner (GP) on suspicion of cardiac disease between 2007 and 2018. During the first visit, information was collected on anthropometric measurements, cardiovascular risk factors, medical history, comorbidities and medication use. All patients underwent transthoracic echography. 
For the current study, all patients with a new HF diagnosis registered in the clinical care database within the two weeks prior to and including the baseline CCN visit ('de novo' HF) were included. As all patients referred to $\mathrm{CCN}$ are seen within two weeks, any diagnosis within this period can be considered de novo. Patients with known HF were excluded to minimise survival bias.

HF was defined as either (i) a diagnosis of heart failure or decompensatio cordis registered by the cardiologist, or (ii) a diagnosis of left ventricular dysfunction or diastolic dysfunction registered by the cardiologist that was verified using the left ventricular systolic or diastolic function reported by the echocardiographist, respectively. Patients for whom the left ventricular systolic or diastolic function could not be verified were excluded. HF was divided into subtypes according to the guidelines ${ }^{5}$, which were reduced $\mathrm{EF}(\mathrm{EF}<40 \%)$, midrange $\mathrm{EF}$ (40-50\%), and preserved EF ( $\geq 50 \%)$.

\section{Guideline-recommended HF medication and target dose}

Guideline-recommended medication groups were defined based on the 2016 ESC HF guidelines ${ }^{5}$ and included ACEls and ARBs, beta-blockers, and mineralocorticoid receptor antagonists (MRAs).

Medication prescriptions were identified with pattern matching based on a combination of generic compound names and brand names. Medication dose was extracted from the text fields using pattern matching and multiplied by daily frequency and dosage to obtain the daily dose per prescription.

Target doses were taken from the 2016 ESC HF guidelines ${ }^{5}$. For medications not included in the guideline, target doses were taken from the literature " or the doses were converted to another medication within the same class that had target dose available using conversion tables $^{13-15}$. To enable comparison across medications and medication groups, daily dose was converted to percentage of target dose. Percentage of target dose was used both as continuous and dichotomous variable with levels $<50 \%$ and $\geq 50 \%$, depending on the analysis. The dichotomous variable was chosen based on the hypothesis that women have a better prognosis at $<50 \%$ of target dose.

Baseline HF medication was defined as the first prescription for each medication category that was prescribed within the period 31 days prior to and 31 days after the patient's $\mathrm{CCN}$ visit. First prescriptions that started either before or after the relevant time window, prescriptions that ended before the $\mathrm{CCN}$ visit, one-off prescriptions, and prescriptions with data entry errors were excluded. As patients could have prescriptions from several medication groups, excluding first prescriptions from one medication group would not necessarily exclude a patient from the study altogether. Prescriptions started more than $3 \mathrm{I}$ days prior to the $\mathrm{CCN}$ visit were categorised as medication history. Medication use was tracked over time within the CCN clinical care database, including dose changes. To show 
dosage over time, all prescriptions registered after the baseline HF medication prescription were numbered in order of prescription date and the dose was converted to percentage of target dose as described above.

\section{Outcomes}

Passive follow-up for all-cause mortality was available for $95.9 \%$ of the study population via linkage to the national causes of death registry from Statistics Netherlands. This registry continuously collects all official cause of death reports submitted by medical doctors and coroners in the Netherlands and is updated quarterly throughout the year and at the end of each year. The cause of death is coded using the International Classification of Diseases and related Health Problems edition 10 (ICD-I0). For the current analyses, all-cause mortality was available until 12 February 2020. Follow-up time for patients who were still alive was censored at 12 February 2020.

\section{Statistical analyses}

Continuous variables were reported as mean (standard deviation) or median [interquartile range], depending on their distribution. Categorical variables were reported as number and percentage. Sex-specific dosing patterns were evaluated by plotting the percentage of target dose prescribed against consecutive number of prescriptions within each medication group.A natural cubic spline with three knots was used to visualise trends. The sex-specific relationship between percentage of target dose at baseline and all-cause mortality was evaluated using Cox regression and the percentage of target dose categories in the whole population and stratified by HF subtype. The highest dose group was taken as the reference group. We used the product of target dose and sex as an interaction term to test whether observed sex differences in the association between target dose and mortality were statistically significant. We used restricted cubic splines to explore possible non-linearity in the sex-specific relationship between percentage of target dose and all-cause mortality. The number of knots was determined using the Akaike Information Criterion (AIC). To account for confounding by indication, the analyses were inversely weighted with the probability of receiving a dose $\geq 50 \%$. This probability was calculated as a propensity score and included variables that were related to both prescription behaviour and all-cause mortality or allcause mortality only, as recommended in literature ${ }^{16}$. This resulted in more stable propensity scores and inverse probability weights. We calculated propensity scores for each medication group separately, as not all patients were prescribed similar doses in the different medication categories. Missing data on components of the propensity score were imputed with multiple imputation using the mice package ${ }^{17}$ before calculating the propensity scores using the ipw package ${ }^{18}$. Patients whose EF was not recorded (7\%) were excluded from HF subtype analyses. 


\section{Sensitivity analyses}

We performed two subgroup analyses to test the robustness of our findings across patient subgroups, one including only patients for whom the cardiologist wrote down a diagnosis of heart failure or decompensatio cordis (heart failure definition (i)) and one re-defining HFpEF as an $\mathrm{EF} \geq 40 \%$ to include patients with a midrange EF. We also performed five additional analyses to test the robustness of our findings across different categorisations of the exposure. The first used exposure categories based on literature" ", the second used tertiles of the target dose based on the distribution in the cohort, the third used quartiles, and the fourth the last recorded dosage for each patient with the same exposure groups as the main analysis. As dosage was left-skewed, we used the lowest exposure group as reference for the three sensitivity analyses with different exposure categories to obtain more stable estimates. The fifth used binary categories of $\leq 50 \%$ and $>50 \%$ of target dose. To maintain sufficient power, these five sensitivity analyses were performed in the population comprising all HF subtype subgroups.

All analyses were performed in R (R Core Team, Vienna, Austria). A p-value of $<0.05$ was considered statistically significant.

\section{Results}

\section{Study population}

Out of the $38 \mathrm{II} \mathrm{HF} \mathrm{patients} \mathrm{in} \mathrm{the} \mathrm{CCN} \mathrm{database,} \mathrm{II} 76$ patients met our criteria for both de novo HF and use of HF medication (Figure I). In total, 934 patients received an ACEI/ ARB, 795 a beta-blocker and I78 an MRA (Figure I). Thirty-five percent of HFrEF patients ( $n=160)$ had an $E F \leq 30 \%$.

Women comprised $48 \%$ of the study population $(n=56 \mathrm{I})$ and were on average older than men ( $71 \pm 12$ vs $67 \pm 12$, respectively). They more often had hypertension ( $55 \%$ vs $45 \%$ ) and were more often prescribed diuretics (58\% vs $51 \%$ ) than men. However, the prevalence of diabetes ( $13 \%$ vs $20 \%$ ), coronary heart disease ( $8 \%$ vs $19 \%$ ) and previous coronary interventions ( $8 \%$ vs $23 \%$ ) was lower in women compared with men. Women more often presented with HFpEF ( $49 \%$ vs $25 \%$ ) and less often with HFrEF ( $29 \%$ vs $47 \%$ ) than men (Table I).

\section{Sex-specific current dosing practice}

At baseline, the distribution of prescriptions across the three medication groups was similar for both sexes. Seventy six percent of women and $82 \%$ of men received ACEI/ ARBs. These percentages were $68 \%$ and $67 \%$ for beta-blockers and $13 \%$ and $17 \%$ for MRAs, respectively (Table I). Over time, the I I 76 patients in our cohort received 2768 medication prescriptions (46\% for women) and on average each patient had I.5 prescriptions. Only I5\% of prescriptions were given at $\geq 100 \%$ target dose in both sexes. The median dose for all 
three medication categories was similar between the sexes (Table 2). Seventy nine percent of women vs $86 \%$ of men with HFrEF received ACEI/ARBs, compared with $77 \%$ vs $81 \%$ in $\mathrm{HFpEF}$, respectively. For beta-blockers these percentages were $78 \%$ vs $75 \%$ for $\mathrm{HFrEF}$ and $60 \%$ vs $45 \%$ for HFpEF, respectively (Supplementary Table I).

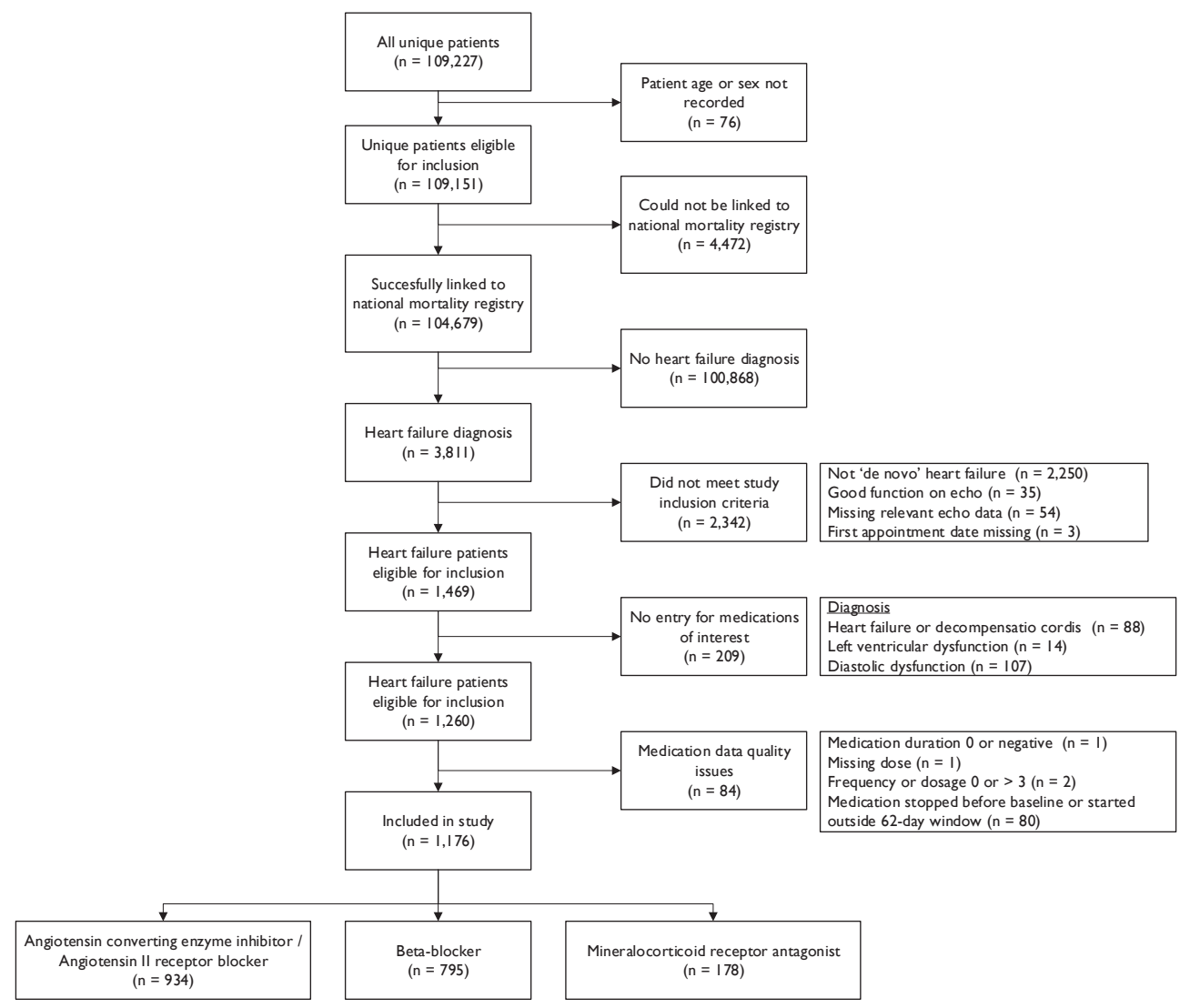

Figure I | Overview of the patient selection process

The natural cubic spline analysis showed that the average dose over consecutive ACEI/ARB and beta-blocker prescriptions remained stable around $50 \%$ of target dose for both sexes, despite some individual patients having dosages of $\geq 100 \%$ target dose. MRA prescriptions did show an upward trend in both sexes and reached $100 \%$ after three or four consecutive prescriptions (Figure 2).

Relationship between target dose and all-cause mortality

Median follow-up time was 3.7 years [2.5-5.9] and 252 patients ( $48 \%$ women) died during follow-up, with cardiovascular disease being the leading cause of death (49\%). Due to the low number of MRA prescriptions, the survival analyses were restricted to ACEl/ARBs and 
beta-blockers. The number of women and men included in each analysis can be found in Supplementary Table I. The interaction terms assessing the interaction between sex and dose were not statistically significant, and neither were the restricted cubic spline analyses.

Table I | Baseline characteristics of the study population

\begin{tabular}{|c|c|c|}
\hline & $\begin{array}{l}\text { Women } \\
(n=561)\end{array}$ & $\begin{array}{c}\text { Men } \\
(n=615)\end{array}$ \\
\hline \multicolumn{3}{|l|}{ General } \\
\hline Age (years) & $7 I(12)$ & $67(12)$ \\
\hline \multicolumn{3}{|l|}{ Ethnicity (n, \%) } \\
\hline Native Dutch & 47I (84.0) & 478 (77.7) \\
\hline First generation immigrant & $54(9.6)$ & $88(14.3)$ \\
\hline Second generation immigrant & $36(6.4)$ & $49(8.0)$ \\
\hline Income $(€)$ & $17,435[10,733-26,39 \mid]$ & $32,35 \mid[18,545-46,647]$ \\
\hline Current smoker (n, \%) & $158(28.2)$ & $167(27.2)$ \\
\hline \multicolumn{3}{|l|}{ Ejection fraction (n, \%) } \\
\hline$<40 \%$ & $162(28.9)$ & 291 (47.3) \\
\hline $40-49 \%$ & $86(15.3)$ & $123(20.0)$ \\
\hline$\geq 50 \%$ & $272(48.5)$ & $155(25.2)$ \\
\hline not recorded & $41(7.3)$ & $46(7.5)$ \\
\hline \multicolumn{3}{|l|}{ Medical history and comorbidities (n, \%) } \\
\hline Hypertension & $309(55.1)$ & $274(44.6)$ \\
\hline Diabetes mellitus & $74(13.2)$ & $122(19.8)$ \\
\hline Coronary heart disease & $42(7.5)$ & $118(19.2)$ \\
\hline Cerebrovascular disease & $47(8.4)$ & $52(8.5)$ \\
\hline Cardiovascular intervention & $47(8.4)$ & $140(22.8)$ \\
\hline Arrhythmia & II 6 (20.7) & $154(25.0)$ \\
\hline Valvular heart disease & $50(8.9)$ & $62(10.1)$ \\
\hline \multicolumn{3}{|l|}{ Clinical measures } \\
\hline Body mass index $\left(\mathrm{kg} / \mathrm{m}^{2}\right)$ & $27.5(5.6)$ & $28.1(4.7)$ \\
\hline Systolic blood pressure (mmHg) & $150(26)$ & $146(24)$ \\
\hline Diastolic blood pressure $(\mathrm{mmHg})$ & $88(14)$ & $88(15)$ \\
\hline Total cholesterol (mmol/L) & $5.02(1.13)$ & $4.55(1.13)$ \\
\hline Triglycerides (mmol/L) & $1.40[1.00-2.10]$ & $1.60[1.10-2.20]$ \\
\hline Estimated glomerular filtration rate $\left(\mathrm{mL} / \mathrm{min} / 1.73 \mathrm{~m}^{2}\right)$ & $65(27)$ & $66(28)$ \\
\hline \multicolumn{3}{|l|}{ Medication prescribed at baseline $(n, \%)$} \\
\hline ACE inhibitor/AR blocker & $428(76.3)$ & $506(82.3)$ \\
\hline Beta-blocker & $38 I(67.9)$ & $414(67.3)$ \\
\hline Mineralocorticoid receptor antagonist & $74(13.2)$ & $104(16.9)$ \\
\hline \multicolumn{3}{|l|}{ Number of medication classes prescribed } \\
\hline $\mathrm{I}$ & $26 \mathrm{I}(46.5)$ & $25 I$ (4I.I) \\
\hline 2 & $25 \mathrm{I}(44.7)$ & $286(46.5)$ \\
\hline 3 & 49 (8.7) & $76(12.4)$ \\
\hline
\end{tabular}

$\mathrm{ACE}=$ angiotensin-converting enzyme, $\mathrm{AR}$ = angiotensin II receptor 
Table 2 | Characteristics of all medication prescriptions in the study population

\begin{tabular}{|lcc|}
\hline & $\begin{array}{c}\text { Women } \\
(\mathbf{n}=\text { I 276) }\end{array}$ & $\begin{array}{c}\text { Men } \\
(\mathbf{n}=\text { I 492) }\end{array}$ \\
\hline Medication group (n, \%) & $619(48.5)$ & $724(48.5)$ \\
ACE inhibitor/AR blocker & $571(44.7)$ & $644(43.2)$ \\
Beta-blocker & $86(6.7)$ & $124(8.3)$ \\
Mineralocorticoid receptor antagonist & & $654(43.8)$ \\
Target dose group (n, \%) & $564(44.2)$ & $608(40.8)$ \\
I-49\% & $513(40.2)$ & $230(15.4)$ \\
$50-99 \%$ & $199(15.6)$ & $50.00[25.00,57.10]$ \\
I00\% or higher & & $25.00[25.00,50.00]$ \\
Median target dose baseline prescriptions $[$ IQR $]$ & $50.00[25.00,66.70]$ & $50.00[50.00,50.00]$ \\
ACE inhibitor/AR blocker & $25.00[25.00,50.00]$ & \\
Beta-blocker & $50.00[50.00,50.00]$ & \\
Mineralocorticoid receptor antagonist & &
\end{tabular}

$\mathrm{ACE}=$ angiotensin-converting enzyme, $\mathrm{AR}=$ angiotensin II receptor

\section{Angiotensin-converting enzyme inhibitors / Angiotensin II receptor blockers}

In all HF patients, the inverse-probability weighted mortality risk was similar for the $<50 \%$ target dose group and the reference group $(H R=1.01,95 \% \mathrm{Cl} 0.76-1.33)$. The results were the same for women and men (Table 3). In the HFrEF subgroup, the mortality risk was lower for the $<50 \%$ target dose group. This was statistically significant in the whole cohort (HR $=0.63,95 \% \mathrm{Cl} 0.42-0.96)$ and in women $(\mathrm{HR}=0.49,95 \% \mathrm{Cl} 0.25-0.99)$ but not in men $(\mathrm{HR}$ $=0.76,95 \% \mathrm{Cl} 0.45-\mathrm{I} .28)$. In the HFpEF subgroup, the mortality risk was not significantly different between the $<50 \%$ target dose group and the reference group $(\mathrm{HR}=1.02,95 \% \mathrm{Cl}$ 0.57-1.84). The restricted cubic spline suggested women had lower mortality risk at lower target doses, whereas men had lower mortality risk at higher target doses (Figure 3A and 3C; Supplementary Figure IA).

\section{Beta-blockers}

In all HF patients, there was no difference in inverse-probability weighted mortality risk between the $<50 \%$ target dose group and the reference group in the whole population (HR $=1.05,95 \% \mathrm{Cl} 0.78-\mathrm{I} .4 \mathrm{I}$ ). Results were similar in women and men separately (Table 3). There was no association between dosage and all-cause mortality in $\mathrm{HFrEF}(\mathrm{HR}=0.99,95 \% \mathrm{Cl}$ 0.62-1.59) or in HFpEF (HR = I.27, 95\% Cl 0.69-2.35). The restricted cubic spline suggested women had lower mortality risk at both extremes of the target dose spectrum, whereas men had lower mortality risk at intermediate doses (Figure 3B and 3D; Supplementary Figure IB). 


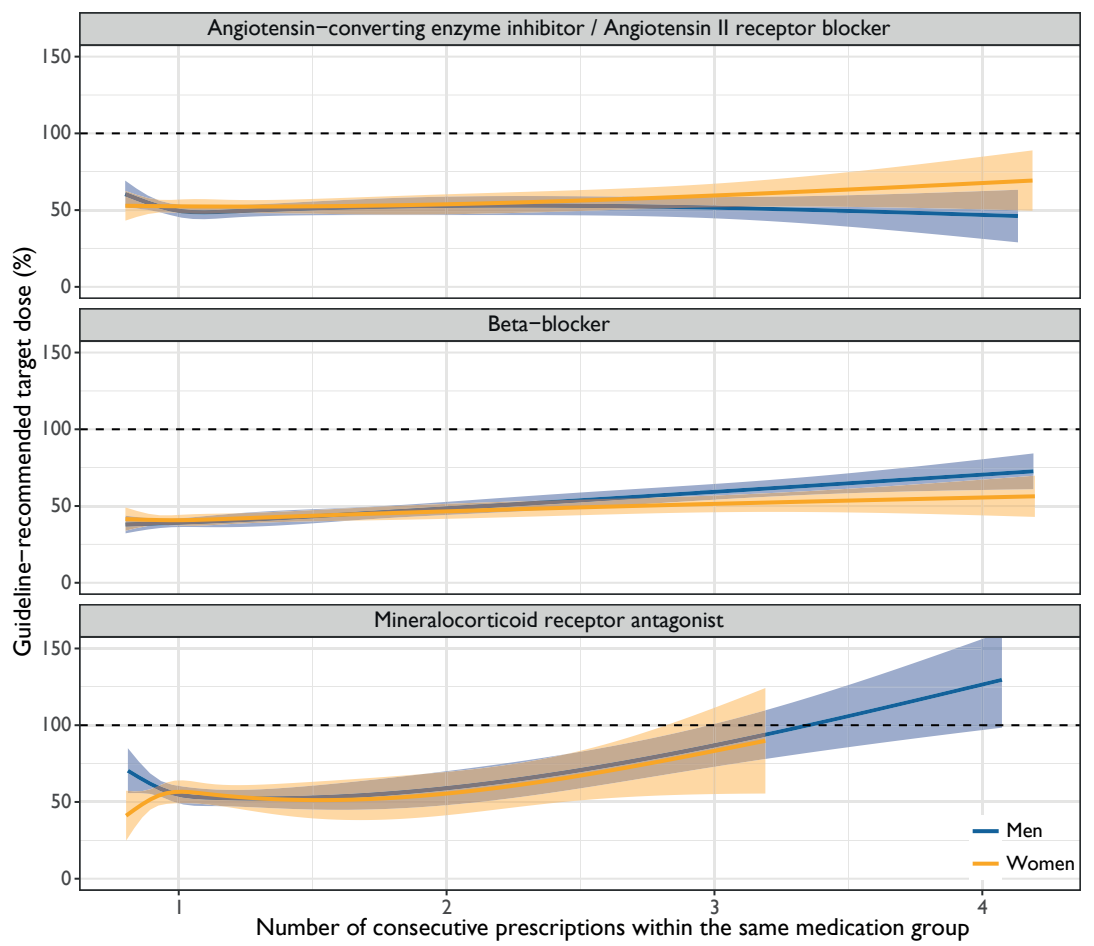

Figure 2 | Percentage of target dose across consecutive prescriptions

\section{Sensitivity analysis}

Performing our analysis in the subgroup of patients with a diagnosis of heart failure or decompensatio cordis $(n=814)$ did not significantly change our results and neither did using the broader definition of HFpEF (Supplementary Table 2, Supplementary Figure 2). The five different target dose categories showed similar results to our main analyses, except for the cohort-based tertiles which showed a statistically significantly higher mortality risk in the intermediate beta-blocker dosage group (50-75\%) in the whole cohort $(\mathrm{HR}=1.70,95 \% \mathrm{Cl}$ $\mathrm{I} .18-2.44)$ and women $(\mathrm{HR}=2.25,95 \% \mathrm{Cl} \mathrm{I} .3 \mathrm{I}-3.38)$ but not in men $(\mathrm{HR}=1.29,95 \% \mathrm{Cl}$ 0.78-I.2I) (Supplementary Table 3).

\section{Discussion}

The majority of HF patients seen in cardiology outpatient clinics received $50 \%$ of the guideline-recommended medication dose. Lower ACEI/ARB dose was associated with the best survival outcomes in women with HFrEF but not men. Beta-blocker dosage was not associated with survival in HFrEF patients. There was no association between medication dosage and survival in HFpEF. 

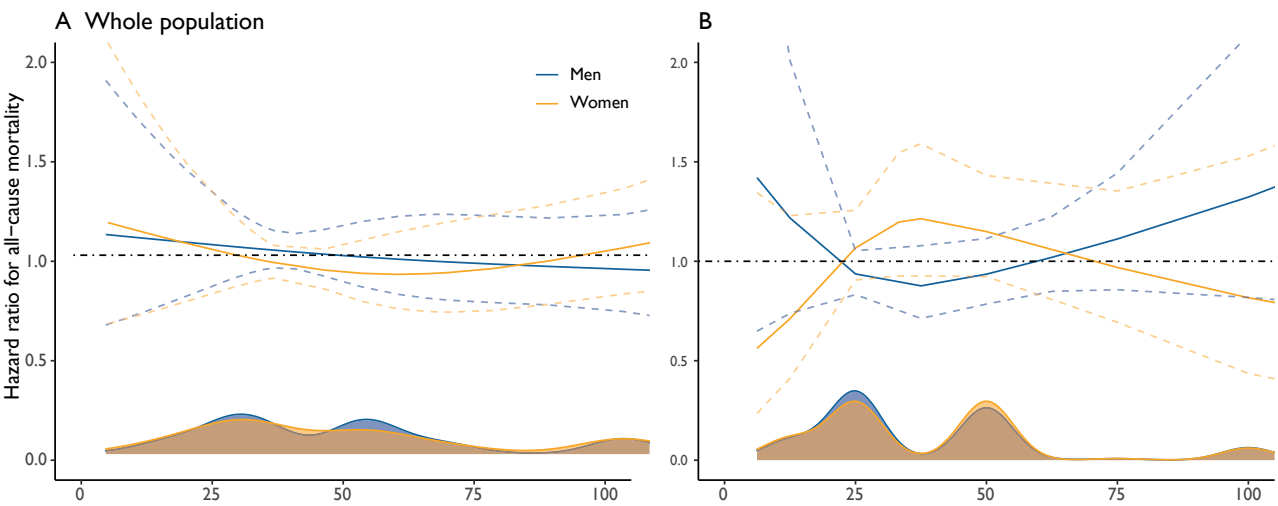

C Heart failure with reduced ejection fraction
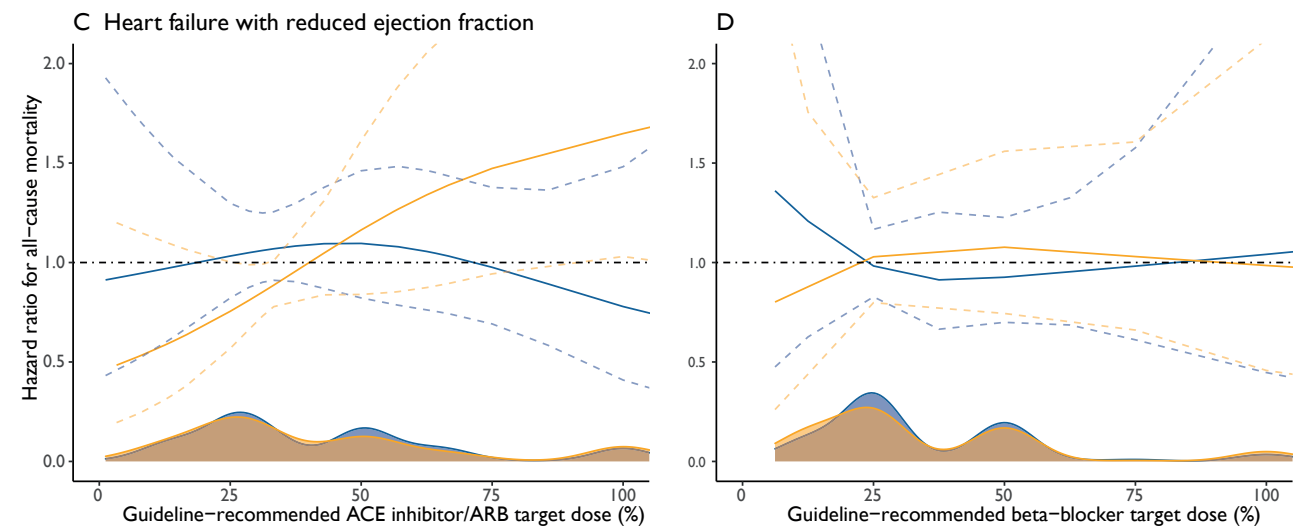

Figure 3 | Sex-specific relationship between percentage of target dose and all-cause mortality risk for ACE-inhibitors / ARBs in all HF subtypes (A) and HFrEF (C), and beta-blockers in all HF subtypes (B) and HFrEF (D)

The distribution of percentage of target dose in the population is given by the density plots on the bottom. The coloured solid lines represent the estimated hazard ratio across the range of target dose and the coloured dashed lines the $95 \%$ confidence interval. The black dashed line shows the line of no effect $(H R=1)$. The cubic spline is by default linear before the first knot, which may be interpreted as no dose (target dose $0 \%$ ) being better than any dose. However, this is an artefact of the cubic spline and thus should not be interpreted as suggesting no dose is better than any dose.

The main strength of our study is the large outpatient cardiology dataset that better reflects current practice and covers a wide range of HF patients with respect to sex, co-morbidities and HF subtype, including patients with mild HF that are not often recruited for studies ${ }^{19}$. The prevalence of HF in the clinical care population seen at CCN is low (3.5\%) and the HF patients included in our study seem to be healthier than other HF populations based on their mortality rate, which was $21 \%$ over a median follow-up of 3.7 years. This is much lower than the $52 \%$ five-year mortality observed in a recent population-based cohort from the United Kingdom ${ }^{20}$ and the $17 \%$ one-year mortality seen across European patients hospitalised for $\mathrm{HF}^{2 \mathrm{l}}$. 


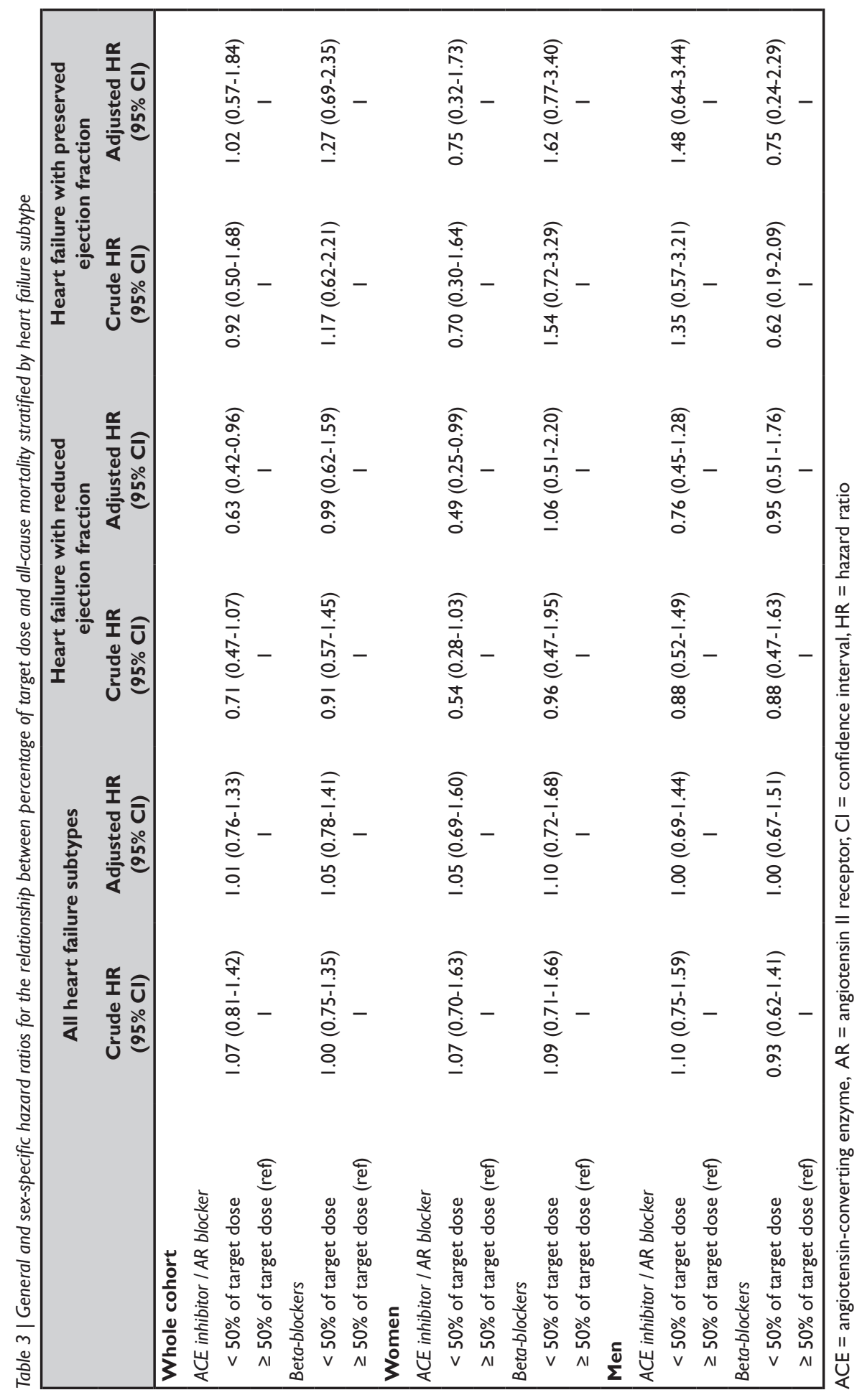


The main limitation of our study is that data collection and active follow-up were driven by medical need and thus not performed systematically. Information on medication trajectory was therefore not complete for each patient, so we could not adjust for changes in regimen in our analyses. Despite our efforts to control for indication bias through inverse probability weighting, residual confounding remains likely and we cannot conclude our observations are causal.We addressed the low number of HFmrEF patients in a sensitivity analysis, which did not change our findings. We could not include a comparison with 'no dose' patients because patients not receiving the medications of interest were excluded to reduce risk of bias. We could not evaluate the effects of specific medication or dose combinations, and could not include sacubitril/valsartan in our analysis because it was not indicated for 'de novo' HF in Dutch guidelines. We were unable to obtain follow-up through record linkage for outcomes such as HF hospitalisation, quality of life and adverse drug reactions (ADRs). Gender was not specifically collected, but we did not observe any significant differences between women and men in prescription behaviour in our cohort.

We found that women with HFrEF receiving $<50 \%$ of the guideline-recommended ACEI/ ARB target dose had a better prognosis than women receiving higher doses. The cubic spline analysis also suggested women might do better on lower dosages while men may have a better prognosis on higher dosages. These results are in line with a previous study reporting significant sex differences in the relationship between medication dosage and a composite outcome of all-cause mortality and HF hospitalisation, where women with HFrEF seemed to have the best prognosis at $50 \%$ of target dose for ACEI/ARBs whereas men with HFrEF required the full dose" ${ }^{\prime}$. Literature has not identified definite sex differences in pharmacokinetics and -dynamics for this medication group ${ }^{22}$, although pharmacogenomic data suggest the risk of ACEl-specific side effects such as cough may be influenced by sex ${ }^{23}$. This remaining uncertainty about potential biological mechanisms underlying our observations, however, does not take away the fact that our findings support the hypothesis that sexspecific ACEI/ARB target doses might benefit HFrEF patients. Prospective dose-finding trials are needed to confirm this and identify the exact optimal dosage. Importantly, our findings are based on HF patients with above average survival situated between the GP and the hospital, so they may not be generalisable to patients with more severe HF.

In contrast to previous work", we did not find any significant association between betablocker dosage and mortality risk in HFrEF patients. This was surprising because women have a lower volume of distribution and slower clearance for this medication group, which results in higher beta-blocker concentrations in the blood when women are given the same dose as men ${ }^{22}$. This would support sex-specific dosing, also because these unintentional higher doses may lead to adverse drug reactions that negatively affect medication adherence and thus long-term prognosis ${ }^{24}$. In this light it is also interesting that the cubic spline analyses for beta-blockers showed a trend opposite to the previous study, with women doing better at higher doses and men doing better at intermediate doses. This might be an artefact due 
to the lower sample size, or because our correction for indication bias was insufficient due to residual confounding.

Alternatively, the low prevalence of prior cardiovascular disease and other co-morbidities in our HF population may explain the lack of association between dosage and all-cause mortality. Data on the effectiveness of guideline-recommended treatment in relatively healthy HF patients treated by a GP are scarce ${ }^{19}$. A meta-analysis of HF trials showed that treatment with ACEls, beta-blockers and MRAs reduced mortality risk equally for all NYHA classes $^{25}$. However, the only trial that focussed specifically on patients with mild HF (defined here as patients with asymptomatic LV dysfunction) did not show a significant difference in all-cause mortality between the placebo and the treatment $\mathrm{arm}^{26}$. ACEl treatment did reduce HF-related hospitalisations in those with mild HF and the incidence of HF in those with reduced EF but no symptoms ${ }^{26}$, suggesting all-cause mortality might not be the most clinically relevant outcome in our HF population. Future work focussing on the association between medication dosage and HF hospitalisations or quality of life-related outcomes may better support first-line healthcare professionals in their medication dosage choices.

Importantly, none of the studies included in this meta-analysis included HFpEF patients ${ }^{25}$ and effective therapies for this HF subtype are still lacking ${ }^{5}$. Guideline-recommended medications for HFrEF are not expected to improve prognosis in HFpEF patients, which explains the lack of association between medication dosage and mortality observed in our HFpEF patients. We also showed that about $80 \%$ of women and men with $\mathrm{HFpEF}$ received ACEI/ARBs, and $60 \%$ of women and $45 \%$ of men were prescribed beta-blockers. This is in line with the guideline recommendation to prescribe these medication types in $\mathrm{HFpEF}$ patients to reduce co-morbidity burden 5 .

Adverse drug reactions (ADRs) are an important component of quality of life related to medication dosage. ADR risk varies both between women and men and across the spectrum of HF due to inherent biological differences and physiological changes that occur as HF progresses ${ }^{22,27}$. Treating physicians may choose not to increase dose if this only results in a higher ADR risk, especially when the benefit of a higher dose is not readily apparent. This may explain why only $15 \%$ of all prescriptions in our study population were given at $\geq 100 \%$ target dose and why we did not see uptitration for ACEI/ARBs and beta-blockers over time. In a previous study only $25 \%$ of ACEI/ARB treated patients and $14 \%$ of beta-blocker treated patients reached target dose after an uptitration phase of three month " $^{\prime}$. Similarly, in a large HFrEF registry approximately $25 \%$ of ACEI/ARB prescriptions and $45 \%$ of betablocker prescriptions were given at less than $50 \%$ of target dose ${ }^{12}$. Data on the (sex-specific) relationship between HF medication dosage and ADRs is still scarce, partially due to poor reporting ${ }^{28,29}$, but also because $A D R s$ are rarely recorded in the electronic health record. 
Better quality sex-specific data are needed to evaluate whether ADRs are indeed one of the main reasons for not up-titrating HF medication and whether a sex-specific approach to dosage could alleviate this issue.

\section{Conclusion}

The majority of women and men with HF seen at cardiology outpatient clinics received half of the guideline-recommended medication dose. This dose was associated with improved survival in women with HFrEF for ACEI/ARBs but not for beta-blockers. Dose was not associated with survival in HFpEF patients regardless of sex. These results underscore the need for dose-finding trials to (re)define optimal medical therapy for women with HFrEF.

\section{Acknowledgements}

Results based on calculations by the authors using non-public microdata from Statistics Netherlands.

\section{Funding}

This work was supported by the Dutch Cardiovascular Alliance consortium DCVA IMPRESS (2020B004) and ERC Consolidator grant UCARE (866478).

\section{Disclosures}

GAS and IIT are employed by Cardiology Centers of the Netherlands. FWA is supported by UCL Hospitals NIHR Biomedical Research Centre. The other authors declare no conflict of interest. 


\section{References}

I. Savarese G, Lund LH. Global Public Health Burden of Heart Failure. Card Fail Rev 2017; 3(I): 7-II.

2. Scott PE, Unger EF, Jenkins MR, et al. Participation of Women in Clinical Trials Supporting FDA Approval of Cardiovascular Drugs. Journal of the American College of Cardiology 2018; 7I(18): 1960-9.

3. Dunlay SM, Roger VL, Redfield MM. Epidemiology of heart failure with preserved ejection fraction. Nature Reviews Cardiology 2017; I4(10): 591-602.

4. Soldin OP, Mattison DR.Sex Differences in Pharmacokinetics and Pharmacodynamics. Clinical Pharmacokinetics 2009; 48(3): 143-57.

5. Ponikowski P,Voors AA, Anker SD, et al. 2016 ESC Guidelines for the diagnosis and treatment of acute and chronic heart failure: The Task Force for the diagnosis and treatment of acute and chronic heart failure of the European Society of Cardiology (ESC)Developed with the special contribution of the Heart Failure Association (HFA) of the ESC. European Heart Journal 2016; 37(27): 2129-200.

6. Pilote L, Raparelli V. Participation of Women in Clinical Trials: Not Yet Time to Rest on Our Laurels. Journal of the American College of Cardiology 2018; 7I(18): 1970-2.

7. Dunlay SM, Roger VL, Redfield MM. Epidemiology of heart failure with preserved ejection fraction. Nat Rev Cardiol 2017; 14(10): 591-602.

8. Sobhani K, Nieves Castro DK, Fu Q, Gottlieb RA, Van Eyk JE, Noel Bairey Merz C. Sex differences in ischemic heart disease and heart failure biomarkers. Biol Sex Differ 2018; 9(I): 43-.

9. Harada E, Mizuno Y, Kugimiya F, et al. Plasma Levels of BNP Are Lower in Women Than Men among Patients with HFpEF. Journal of Cardiac Failure 2017; 23(I0): S58.

10. Sharpe N. Clinical Trials and the RealWorld: Selection Bias and Generalisability of Trial Results. Cardiovascular Drugs and Therapy 2002; 16(I): 75-7.

II. Santema BT, Ouwerkerk W, Tromp J, et al. Identifying optimal doses of heart failure medications in men compared with women: a prospective, observational, cohort study. The Lancet 2019; 394(10205): 1254-63.

12. Veenis JF, Rocca HB, Linssen GC, et al. Impact of sex-specific target dose in chronic heart failure patients with reduced ejection fraction. Eur J Prev Cardiol 2020: 2047487320923I85.

13. McMurray J, Packer M, Desai AS, et al. Dual angiotensin receptor and neprilysin inhibition as an alternative to angiotensin-converting enzyme inhibition in patients with chronic systolic heart failure: rationale for and design of the Prospective comparison of ARNI with ACEI to Determine Impact on Global Mortality and morbidity in Heart Failure trial (PARADIGM-HF). European journal of heart failure 2013; 15(9): 1062-73.

14. McMurray JJ, Packer M, Desai AS, et al. Angiotensin-neprilysin inhibition versus enalapril in heart failure. The New England journal of medicine 2014; 37I(II): 993-1004.

15. Shalansky K, RSunderji R, Loewen P, Frighetto L. Drug and Therapeutics Newsletter. CSU Pharmaceutical Sciences 2004; II(3).

16. Elze MC, Gregson J, Baber U, et al. Comparison of Propensity Score Methods and Covariate Adjustment: Evaluation in 4 Cardiovascular Studies. J Am Coll Cardiol 2017; 69(3): 345-57.

17. Buuren Sv, Groothuis-Oudshoorn K. mice: Multivariate imputation by chained equations in R. Journal of statistical software 2010: I-68.

18. van derWal WM, Geskus RB. ipw:An R Package for Inverse Probability Weighting. 20II 20I I; 43(I3): 23.

19. Gollop ND, Ford J, Mackeith $P$, et al. Are patients in heart failure trials representative of primary care populations? A systematic review. BJGP Open 2018; 2(I): bjgpopen I8X101337-bjgpopen I8X.

20. Taylor CJ, Ordóñez-Mena JM, Roalfe AK, et al.Trends in survival after a diagnosis of heart failure in the United Kingdom 2000-2017: population based cohort study. Bmj 2019; 364: 1223.

21. Maggioni AP, Dahlström U, Filippatos G, et al. EURObservational Research Programme: regional differences and I-year follow-up results of the Heart Failure Pilot Survey (ESC-HF Pilot). European journal of heart failure 2013; 15(7): 808-17.

22. Rosano GM, Lewis B, Agewall S, et al. Gender differences in the effect of cardiovascular drugs: a position document of the Working Group on Pharmacology and Drug Therapy of the ESC. Eur Heart J 20I5; 36(40): 2677-80. 
23. Mas S, Gassò P, Alvarez S, et al. Pharmacogenetic predictors of angiotensin-converting enzyme inhibitorinduced cough: the role of ACE, ABO, and BDKRB2 genes. Pharmacogenet Genomics 20I I; 2 I (9): 53 I-8.

24. Leporini C, De Sarro G, Russo E. Adherence to therapy and adverse drug reactions: is there a link? Expert Opin Drug Saf 2014; 13 Suppl I: S4I-55.

25. Miller RJH, Howlett JG, Exner DV, Campbell PM, Grant ADM, Wilton SB. Baseline Functional Class and Therapeutic Efficacy of Common Heart Failure Interventions: A Systematic Review and Meta-analysis. Canadian Journal of Cardiology 2015; $31(6)$ : 792-9.

26. The SOLVD Investigators. Effect of Enalapril on Mortality and the Development of Heart Failure in Asymptomatic Patients with Reduced Left Ventricular Ejection Fractions. New England Journal of Medicine 1992; 327(10): 685-91.

27. Lainscak M, Vitale C, Seferovic P, Spoletini I, Cvan Trobec K, Rosano GMC. Pharmacokinetics and pharmacodynamics of cardiovascular drugs in chronic heart failure. International Journal of Cardiology 2016; 224: $191-8$.

28. Bots SH, den Ruijter HM. Recommended Heart Failure Medications and Adverse Drug Reactions in Women. Circulation 2019; I39(12): 1469-7I.

29. Bots SH, Groepenhoff F, Eikendal ALM, et al. Adverse Drug Reactions to Guideline-Recommended Heart Failure Drugs in Women:A Systematic Review of the Literature. JACC Heart Fail 2019; 7(3): 258-66. 


\section{Supplementary files}

\section{Supplementary Figures}
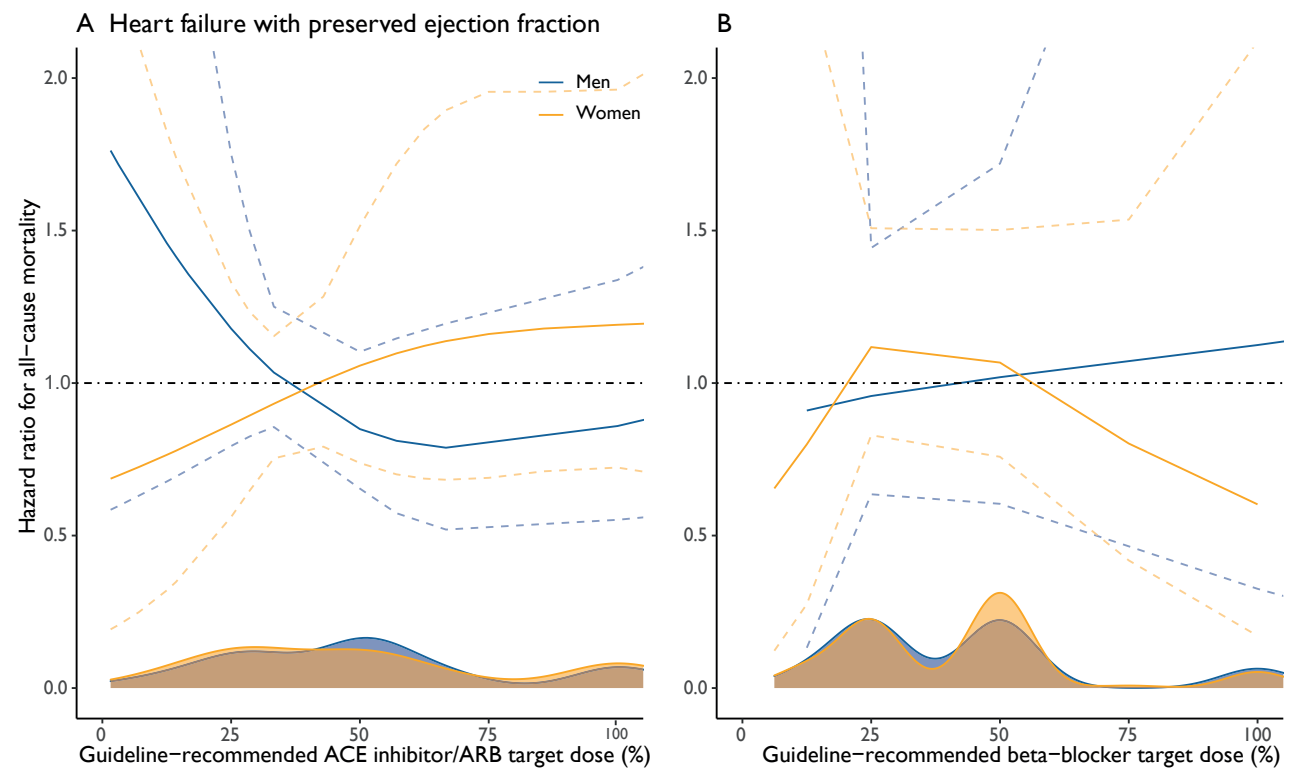

Supplementary Figure I | Sex-specific relationship between percentage of target dose and all cause mortality risk in the subset of patients with heart failure with preserved ejection fraction for ACE inhibitors / ARBs (A) and beta-blockers (B)

The distribution of percentage of target dose in the population is given by the density plots on the bottom. The coloured solid lines represent the estimated hazard ratio across the range of target dose and the coloured dashed lines the $95 \%$ confidence interval. The black dashed line shows the line of no effect $(H R=I)$. The cubic spline is by default linear before the first knot, which may be interpreted as no dose (target dose $0 \%$ ) being better than any dose. However, this is an artefact of the cubic spline and thus should not be interpreted as suggesting no dose is better than any dose. 
A Heart failure specifically mentioned in diagnosis text

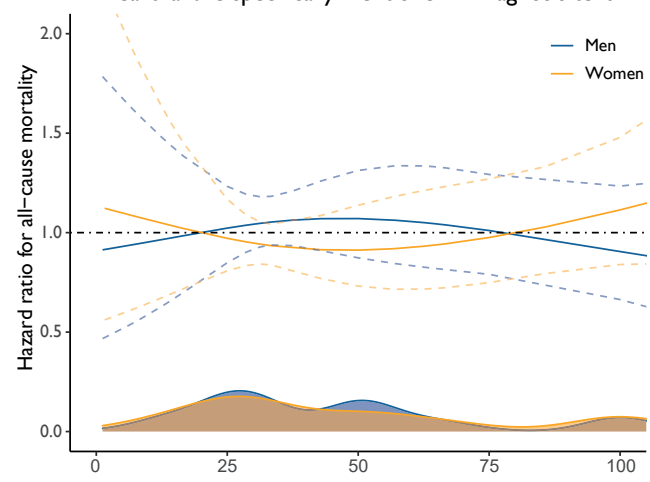

C Last observation carried forward

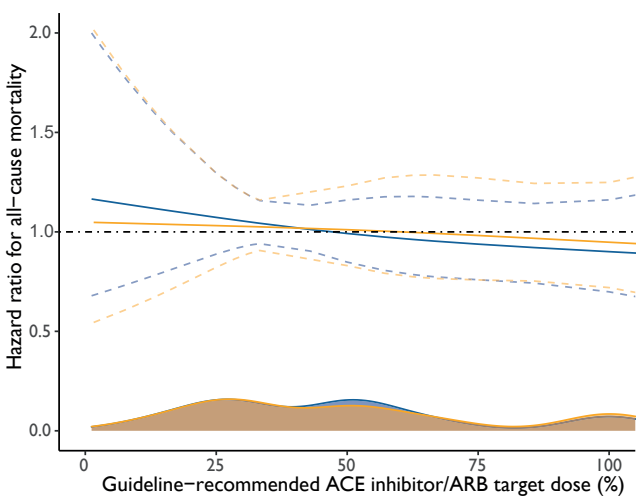

B

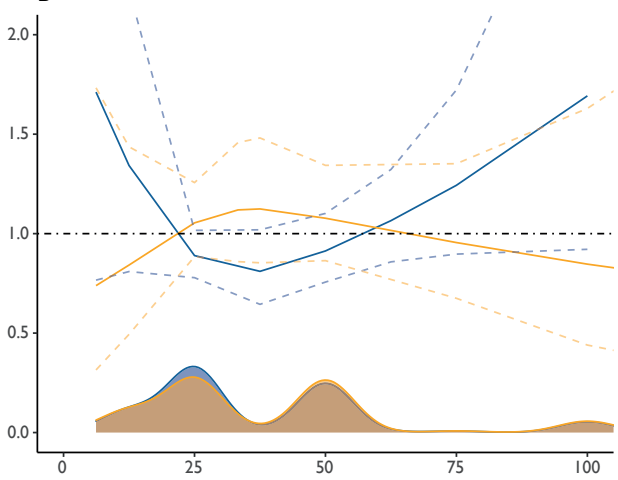

D

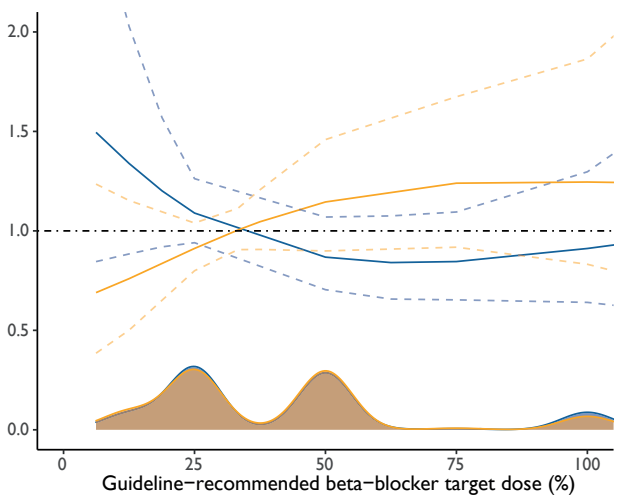

Supplementary Figure 2 | Sex-specific relationship between percentage of target dose and all cause mortality risk in the subset of patients with specific mention of heart failure in their diagnosis text for ACE inhibitors / ARBs (A) and beta-blockers (B), and using last recorded dosage for ACE inhibitors / ARBs (C) and beta-blockers (D)

The distribution of percentage of target dose in the population is given by the density plots on the bottom. The coloured solid lines represent the estimated hazard ratio across the range of target dose and the coloured dashed lines the $95 \%$ confidence interval. The black dashed line shows the line of no effect $(H R=I)$. The cubic spline is by default linear before the first knot, which may be interpreted as no dose (target dose $0 \%$ ) being better than any dose. However, this is an artefact of the cubic spline and thus should not be interpreted as suggesting no dose is better than any dose. 


\section{Supplementary Tables}

Supplementary Table I | Overview of number of women and men included in each analysis based on heart failure subtype and medication group

\begin{tabular}{|lcccc|}
\hline & $\begin{array}{c}\text { Angiotensin-converting enzyme } \\
\text { inhibitors I }\end{array}$ & Beta-blockers \\
& Angiotensin II receptor blockers & & \\
\hline Heart failure subtype & Women & Men & Women & Men \\
\hline Reduced ejection fraction & 128 & 250 & 126 & 219 \\
Midrange ejection fraction & 60 & 93 & 63 & 87 \\
Preserved ejection fraction & 210 & 126 & 162 & 70 \\
Unrecorded ejection fraction & 30 & 37 & 30 & 38 \\
\hline
\end{tabular}

Supplementary Table 2 | General and sex-specific hazard ratios for the relationship between percentage of target dose and all-cause mortality for sensitivity analyses in the subgroup of patients with heart failure specifically mentioned in their diagnosis text, using the last observed medication dosage instead of baseline, and using a different definition of HFrEF

\begin{tabular}{|c|c|c|c|}
\hline & $\begin{array}{l}\text { Heart failure specifically } \\
\text { mentioned in the } \\
\text { diagnosis text }\end{array}$ & $\begin{array}{l}\text { Last observation } \\
\text { carried forward }\end{array}$ & $\begin{array}{l}\text { Heart failure with } \\
\text { preserved ejection } \\
\text { fraction ( } E F \geq 40 \% \text { ) }\end{array}$ \\
\hline & $\begin{array}{l}\text { Adjusted HR } \\
(95 \% \mathrm{Cl})\end{array}$ & $\begin{array}{l}\text { Adjusted HR } \\
\qquad(95 \% \mathrm{Cl})\end{array}$ & $\begin{array}{l}\text { Adjusted HR } \\
\qquad(95 \% \mathrm{Cl})\end{array}$ \\
\hline \multicolumn{4}{|l|}{ Whole cohort } \\
\hline \multicolumn{4}{|c|}{ Angiotensin-converting enzyme inhibitors/Angiotensin II receptors } \\
\hline$<50 \%$ of target dose & $0.82(0.60-1.12)$ & $\mathrm{I} .03(0.78-\mathrm{I} .37)$ & $0.72(0.47-1.10)$ \\
\hline$\geq 50 \%$ of target dose (ref) & I & I & I \\
\hline \multicolumn{4}{|l|}{ Beta-blockers } \\
\hline$<50 \%$ of target dose & $1.21(0.88-1.67)$ & $\mathrm{I} .08(0.80-\mathrm{I} .44)$ & $0.91(0.59-1.42)$ \\
\hline$\geq 50 \%$ of target dose (ref) & $\mathrm{I}$ & I & I \\
\hline \multicolumn{4}{|l|}{ Women } \\
\hline \multicolumn{4}{|c|}{ Angiotensin-converting enzyme inhibitors/Angiotensin II receptors } \\
\hline$<50 \%$ of target dose & $0.85(0.53-1.35)$ & $1.05(0.69-1.6 I)$ & $0.61(0.33-1.12)$ \\
\hline$\geq 50 \%$ of target dose (ref) & 1 & I & I \\
\hline \multicolumn{4}{|l|}{ Beta-blockers } \\
\hline$<50 \%$ of target dose & I.3I (0.83-2.07) & $0.87(0.57-1.33)$ & $0.83(0.46-1.49)$ \\
\hline$\geq 50 \%$ of target dose (ref) & I & I & $\mathrm{I}$ \\
\hline \multicolumn{4}{|l|}{ Men } \\
\hline \multicolumn{4}{|c|}{ Angiotensin-converting enzyme inhibitors/Angiotensin II receptors } \\
\hline$<50 \%$ of target dose & $0.82(0.54-1.24)$ & $1.03(0.7 \mid-1.50)$ & $0.84(0.45-1.57)$ \\
\hline$\geq 50 \%$ of target dose (ref) & 1 & I & I \\
\hline \multicolumn{4}{|l|}{ Beta-blockers } \\
\hline$<50 \%$ of target dose & I.II (0.7I-I.75) & I.3I (0.88-I.99) & $1.04(0.53-2.06)$ \\
\hline$\geq 50 \%$ of target dose (ref) & I & I & I \\
\hline
\end{tabular}

$\mathrm{Cl}=$ confidence interval, $\mathrm{HR}=$ hazard ratio 
Supplementary Table 3 | General and sex-specific hazard ratios for the relationship between percentage of target dose and all-cause mortality using three different medication dosage categories

\begin{tabular}{|c|c|c|c|}
\hline & $\begin{array}{c}\text { Literature-based } \\
\text { categories }\end{array}$ & Cohort-based tertiles & Quartiles \\
\hline & $\begin{array}{l}\text { Adjusted HR } \\
(95 \% \mathrm{Cl})\end{array}$ & $\begin{array}{l}\text { Adjusted HR } \\
(95 \% \mathrm{CI})\end{array}$ & $\begin{array}{l}\text { Adjusted HR } \\
(95 \% \mathrm{CI})\end{array}$ \\
\hline \multicolumn{4}{|l|}{ Whole cohort } \\
\hline \multicolumn{4}{|c|}{ Angiotensin-converting enzyme inhibitors/Angiotensin II receptors } \\
\hline First category (ref) & 1 & I & I \\
\hline Second category & $0.98(0.72-1.34)$ & $0.86(0.6 I-I .2 I)$ & I.04 (0.63-I.7I) \\
\hline Third category & I.0I (0.70-I.48) & $0.90(0.64-1.26)$ & I.0I (0.6I-I.68) \\
\hline Fourth category & na & na & I.04 (0.60-I.79) \\
\hline \multicolumn{4}{|l|}{ Beta-blockers } \\
\hline First category (ref) & 1 & I & I \\
\hline Second category & $0.92(0.67-1.26)$ & $1.70(1.18-2.44)$ & $1.29(0.76-2.19)$ \\
\hline Third category & I.II (0.67-I.84) & $0.97(0.65-1.44)$ & I. 10 (0.64-I.90) \\
\hline Fourth category & na & na & I. $43(0.75-2.74)$ \\
\hline \multicolumn{4}{|l|}{ Women } \\
\hline \multicolumn{4}{|c|}{ Angiotensin-converting enzyme inhibitors/Angiotensin II receptors } \\
\hline First category (ref) & 1 & 1 & I \\
\hline Second category & $0.83(0.50-1.40)$ & $0.95(0.56-1.62)$ & $\mathrm{I} .10(0.5 \mathrm{I}-2.36)$ \\
\hline Third category & I.I2 (0.66-I.89) & $\mathrm{I} .05(0.63-1.74)$ & $0.92(0.4 \mid-2.06)$ \\
\hline Fourth category & na & na & $1.16(0.52-2.62)$ \\
\hline \multicolumn{4}{|l|}{ Beta-blockers } \\
\hline First category (ref) & 1 & 1 & 1 \\
\hline Second category & $0.97(0.63-I .5 I)$ & $2.25(1.31-3.88)$ & $2.13(0.91-5.01)$ \\
\hline Third category & $0.62(0.25-1.56)$ & $1.06(0.58-1.96)$ & $1.72(0.72-4.1 \mathrm{I})$ \\
\hline Fourth category & na & na & $1.58(0.54-4.60)$ \\
\hline \multicolumn{4}{|l|}{ Men } \\
\hline \multicolumn{4}{|c|}{ Angiotensin-converting enzyme inhibitors/Angiotensin II receptors } \\
\hline First category (ref) & I & 1 & 1 \\
\hline Second category & $\mathrm{I} .05(0.70-\mathrm{I} .57)$ & $0.79(0.5|-| .22)$ & $1.00(0.52-1.93)$ \\
\hline Third category & $0.90(0.52-I .56)$ & $0.79(0.50-1.24)$ & $\mathrm{I} .04(0.54-2.00)$ \\
\hline Fourth category & na & na & $0.92(0.44-1.94)$ \\
\hline \multicolumn{4}{|l|}{ Beta-blockers } \\
\hline First category (ref) & 1 & 1 & 1 \\
\hline Second category & $0.86(0.55-1.35)$ & $1.29(0.78-2.12)$ & $0.82(0.4|-| .62)$ \\
\hline Third category & $1.61(0.87-2.98)$ & $0.91(0.54-1.52)$ & $0.74(0.37-1.50)$ \\
\hline Fourth category & na & na & I.27 (0.56-2.89) \\
\hline
\end{tabular}

$\mathrm{Cl}=$ confidence interval, $\mathrm{HR}=$ hazard ratio 



\section{Statins reduce mortality}

risk to a greater extent in women compared
with men in a cardiac
outpatient population

7

Sophie H. Bots

N. Charlotte Onland-Moret

Milena Jancev

Monika Hollander

Igor I.Tulevski

Leonard Hofstra

G. Aernout Somsen

Hester M. den Ruijter 


\begin{abstract}
Aims

Uncertainty about the benefit of (high-intensity) statins for women remains due to underrepresentation of women in primary prevention trials and scarcity of sex-stratified data.This study evaluates the sex-specific relation between statin treatment and survival and the additional benefit of high-intensity regimens.
\end{abstract}

\title{
Methods
}

Electronic health record data from 47,80I patients without prior cardiovascular disease were extracted from thirteen Dutch outpatient cardiology clinics. Patients prescribed statins at baseline were propensity-score matched to those eligible for statin therapy (low-density lipoprotein $>2.5 \mathrm{mmol} / \mathrm{L}$ ) without a statin prescription. Statins were divided into low- and high-intensity according to Dutch guidelines. Mortality data were obtained via linkage to the national mortality registry. Cox regression was used to evaluate the relationship between statin prescription and intensity and all-cause and cardiovascular mortality.

\section{Results}

Propensity score matching created a cohort of $863 \mathrm{I}$ statin users and $863 \mathrm{I}$ non-users. Thirtyfive percent of women and $28 \%$ of men received a low-intensity statin. The beneficial effect of statins on both all-cause and cardiovascular mortality was stronger in women $(H R=0.66$, 0.58-0.74 and $H R=0.55,0.39-0.7 I$, respectively) than in men ( $\mathrm{HR}=0.89,0.8 \mathrm{I}-0.95$ and $\mathrm{HR}$ $=0.93,0.77-\mathrm{I} .08$, respectively). High-intensity statins conferred modest protection against all-cause mortality $(H R=0.94,0.88-1.00)$ and cardiovascular mortality $(H R=0.86,0.74-0.98)$ in both sexes.

\section{Conclusion}

The protective effect of primary prevention statins was stronger in women than men for both all-cause and cardiovascular mortality. High-intensity statins conferred a modest additional benefit in both sexes. Statins seem to be effective regardless of treatment intensity, especially in women. 


\section{Introduction}

Statins have been shown to effectively lower the risk of cardiovascular events by reducing lowdensity lipoprotein (LDL) levels, with high-intensity regiments being more effective than lowintensity variants'. This effect is seen independently of baseline mortality risk ${ }^{2}$, suggesting that all people at risk of cardiovascular events benefit from treatment. However, women are less likely to receive guideline-recommended statin therapy than men in both primary and secondary prevention $^{3-5}$. It has been shown that women are more likely to refuse or stop statin therapy ${ }^{3,6}$ and that healthcare professionals are also less likely to prescribe (high-intensity) statins for women ${ }^{3,5}$.This careful approach may stem from remaining uncertainty about the benefit of statin therapy as primary prevention strategy for women due to the low number of women in primary prevention trials ${ }^{7}$.

There is some evidence to suggest that women and men may benefit equally from statin treatment ${ }^{8,9}$. The Justification for the Use of Statins in Prevention:An Intervention Trial Evaluating Rosuvastatin (JUPITER) trial showed that rosuvastatin reduced the risk of cardiovascular events and cardiovascular mortality in both women and men ${ }^{10}$. In addition, data from a primary care cohort of new statin users showed that improved adherence to statins reduced the risk of hospitalisation for cardiovascular events in both men and women to a similar extent ${ }^{6}$. While some meta-analyses of primary prevention trials confirmed that statin treatment benefits both sexes $^{2,10}$, others failed to find such positive effects ${ }^{11,12}$. Moreover, it remains unclear whether the intensity of therapy influences outcomes. Therefore, we used regular care data from cardiac outpatient clinics to study whether receiving a statin prescription for primary prevention affects long-term prognosis in women and men without a history of cardiovascular disease. We also explored whether high-intensity statins confer additional protection over low-intensity regimens.

\section{Methods}

\section{Study population}

The Cardiology Centers of the Netherlands (CCN) regular care database contains data from 109,15I unique patients that were referred for a full cardiac workup by their general practitioner on suspicion of cardiac disease between 2007 and 2018. During the first visit information was collected on anthropometric measurements, cardiovascular risk factors, medical history, comorbidities, and medication use. All patients underwent transthoracic echography and electrocardiography at rest. Stress electrocardiography and laboratory measurements were performed in approximately $75 \%$ of patients. Passive follow-up was obtained for $95.9 \%$ of the study population via linkage to the national mortality registry.

For the current study all patients with a history of coronary heart disease, heart failure, cerebrovascular disease or congenital heart disease, or a prior or ongoing statin prescription were excluded. 


\section{Selection of statin users and non-users}

Medication prescriptions were identified using pattern matching based on a combination of generic compound names and brand names. The search term was based on brand names of statins available in the Netherlands tailored to those that were prescribed within the study population. It contained the words statin, crestor, lipitor, selektine, zocor, and tahor. Medication dose was extracted from the text fields and multiplied by daily frequency and dosage to obtain the daily dose per prescription. The grepl and gsub functions from the R grep package were used for the name and dose pattern matching, respectively. Statin prescription entries missing information on dose or daily frequency, prescriptions with a negative duration, and one-off prescriptions were excluded. Combination preparations with ezetimibe or fenofibrate were also excluded because Dutch guidelines recommend these when prior statin-only therapy was not effective enough, so these prescriptions are unlikely to be a patient's first statin prescription.

Statin users were defined as patients who received their first statin prescription within 31 days prior to or after their baseline visit. The $3 \mathrm{I}$-day window was chosen to both allow for small delays in data entry and include people prescribed statins shortly before or after their visit at the cardiac outpatient clinic, because labelling these people as non-users would introduce misclassification. Non-users were defined as patients eligible for statin therapy according to the Dutch primary prevention guidelines (low-density lipoprotein $>2.5 \mathrm{mmol} / \mathrm{L}$ ) who did not receive a statin prescription within the 3 I-day period.

\section{Definition of statin intensity}

The intensity of statin treatment was categorised as lower, equal, or higher based on how much it lowered LDL cholesterol levels compared to the Dutch primary prevention guidelinerecommended dose of $40 \mathrm{mg}$ simvastatin (Supplementary Table I) ${ }^{13-15}$. We compared patients on lower intensity statin regimens (low-intensity) with patients on equal and higher intensity regimens (high-intensity).

\section{Outcome}

Passive follow-up for all-cause mortality was available for $95.9 \%$ of the study population via linkage to the national mortality registry from Statistics Netherlands. This registry continuously collects all official cause of death reports submitted by medical doctors and coroners in the Netherlands and is updated quarterly throughout the year and at the end of each year. The cause of death is coded according to the guidelines of the World Health Organisation using the International Classification of Diseases and related Health Problems edition 10 (ICD-I0). For the current analyses, all-cause mortality was available until 12 February 2020 and cause-specific mortality was available until I January 2020. Follow-up time for patients who were still alive was censored on I 2 February 2020. All-cause mortality was defined as having a date of death recorded in the mortality registry. Cardiovascular 
mortality was defined as any mortality entry that was labelled with an ICD-I0 code from the diseases of the circulatory system chapter (100-199).

\section{Propensity score matching}

We created propensity-score matched cohorts for our analyses to account for confounding by indication. We first imputed missing data on components of the propensity score using the mice ${ }^{16}$ package, resulting in five imputed datasets. We subsequently calculated the propensity score in each imputed dataset based on a set of confounding variables (Supplementary Table 2) and matched one-on-one to create five new cohorts where all confounders were balanced between the two groups. We used the Matchlt ${ }^{17}$ package to calculate the propensity score and perform the matching, applying the nearest neighbours approach with a caliper width of 0.1 .We assessed whether covariates were properly balanced based on the mean difference using the cobalt package. We then ran our survival analyses in each imputed matched dataset and pooled the results using the meta package.

For the analyses exploring the relationship between statin use and long-term prognosis, we propensity-score matched statin users with statin non-users. For the analyses exploring the relationship between statin intensity and long-term prognosis, we propensity-score matched low-intensity statin users with high-intensity statin users.

\section{Statistical analyses}

Continuous variables were reported as mean (standard deviation) or median [interquartile range], depending on their distribution. Categorical variables were reported as number and percentage. The effect of statin prescription on long-term prognosis and the effect of statin intensity on long-term prognosis were evaluated using Cox regression in the whole propensity-matched cohort and stratified by sex. We calculated robust confidence intervals to account for the matched nature of the dataset. The presence of sex differences in treatment effect was tested using an interaction term.

\section{Sensitivity analysis}

We excluded participants with a history of cardiovascular disease, which was defined as having a cardiovascular diagnosis before baseline. However, 2000 patients (I2\%) were diagnosed with cardiovascular disease at baseline. To test the robustness of our findings, we repeated the main analysis excluding these 2000 patients. A new propensity score matched cohort was created with the same set of variables used in the main analysis except those related to cardiovascular disease diagnosis. We based our definition of high-intensity statin on Dutch guidelines, which resulted in a different classification than what is internationally used. To check generalisability of our results, we repeated the main analysis defining highintensity statins as those that reduce LDL cholesterol by $50 \%$ or more (atorvastatin $40 \mathrm{mg}$ or higher, rosuvastatin $20 \mathrm{mg}$ or higher, and simvastatin $80 \mathrm{mg}$ or higher). 
All analyses were performed in R (R Core Team, Vienna, Austria). A p-value of $<0.05$ was considered statistically significant.

\section{Ethics statement}

The Cardiology Center of the Netherlands data were made available under implied consent and transferred to the University Medical Center Utrecht under the Dutch Personal Data Protection Act. This study used data collected during the regular care process and did not subject participants to additional procedures or impose behavioural patterns on them. The Medical Research Ethics Committee of the University Medical Center Utrecht declared that the Medical Research Involving Human Subjects Act does not apply to this study (proposal number 17/359).

\section{Results}

\section{Propensity score matching}

We extracted data from 17,008 statin users (48\% women) and 30,793 patients $(57 \%$ women) eligible for statin therapy who did not receive any statins (non-users) (Figure I; Supplementary Table 3). Statin users were on average older (62 \pm I I vs $55 \pm 13$ years), more often men ( $52 \%$ vs $43 \%$ ) and had a higher median 10-year cardiovascular mortality risk (3.94 [1.68-8.36] vs I.66 [0.45-4.77]) compared with non-users. They also more often presented with co-morbidities such as hypertension ( $46 \%$ vs $23 \%$ ) and dyslipidaemia ( $39 \%$ vs $7 \%$ ), and were more often prescribed cardiovascular medication other than statins at baseline ( $80 \%$ vs $34 \%$ ) compared with non-users (Supplementary Table 3). Due to these pronounced differences between statin users and non-users, it was not possible to match each statin user to a non-user with a similar propensity score and individuals that could not be matched were excluded from analyses. Propensity-score matching resulted in a cohort consisting of $863 \mathrm{I}$ statin users and $863 \mathrm{I}$ non-users who were highly similar on all baseline characteristics (Table I).

\section{Propensity-score matched study population}

Female statin users were on average older than male statin users (62 \pm 10 vs $59 \pm$ II years) and more often had a low (0-4\%) cardiovascular mortality risk (61\% vs $49 \%)$. They less often presented without cardiovascular complaints (36\% vs $45 \%$ ) and had a lower estimated glomerular filtration rate (eGFR) $\left(76 \pm 24\right.$ vs $\left.89 \pm 28 \mathrm{~mL} / \mathrm{min} / \mathrm{m}^{3}\right)$. Similar differences between the sexes were seen for the statin non-users. The prevalence of comorbidities and medication use was similar between the sexes for both statin users and non-users (Table 2). 


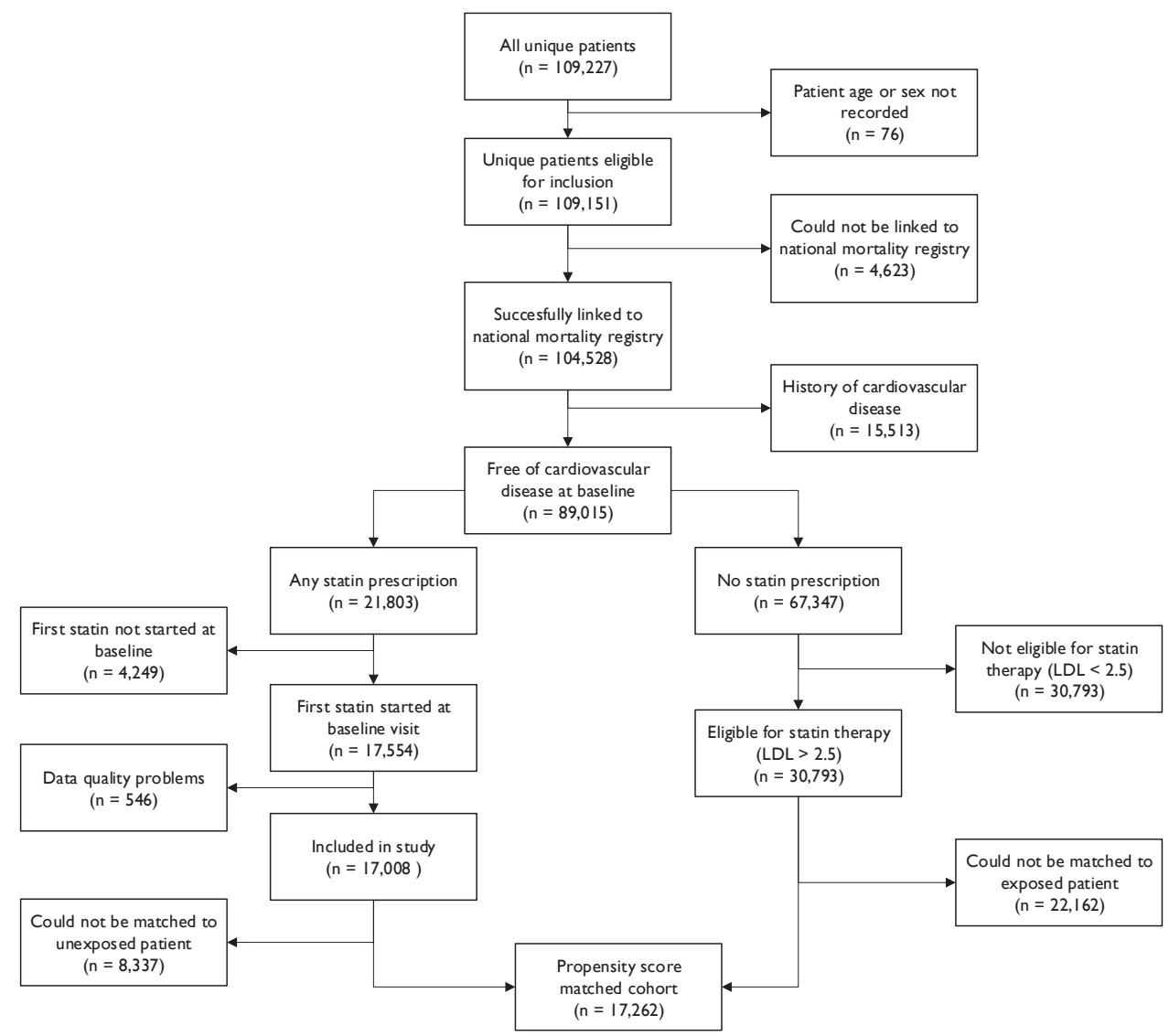

Figure I | Overview of the study population selection process

Statin use and mortality risk

Median follow-up in the matched cohort was 5.8 [3.5-7.9] years, during which 1035 patients ( $50 \%$ women) died. Cardiovascular deaths accounted for 270 events ( $26 \%$ of total). In the whole cohort, statin use reduced the risk of all-cause mortality by $24 \%(\mathrm{HR}=0.76,95 \% \mathrm{Cl}$ $0.7 \mathrm{I}-0.82)$ and the risk of cardiovascular mortality by $28 \%(\mathrm{HR}=0.72,95 \% \mathrm{Cl} 0.6 \mathrm{I}-0.83)$. This protective effect was stronger in women, with a $34 \%$ lower all-cause mortality risk (HR $=0.66,95 \% \mathrm{Cl} 0.58-0.74)$ and a $45 \%$ lower cardiovascular mortality risk $(\mathrm{HR}=0.55,95 \% \mathrm{Cl}$ 0.39-0.7I) in statin users compared with non-users. A similar but weaker trend was seen for men, with an II\% reduction in all-cause mortality $(H R=0.89,95 \% \mathrm{Cl} 0.8 \mathrm{I}-0.96)$ and a non-significant $7 \%$ reduction in cardiovascular mortality $(\mathrm{HR}=0.93,95 \% \mathrm{Cl} 0.77-\mathrm{I} .08)$ in statin users compared with non-users (Table 3). The interaction term for sex was statistically significant for both all-cause mortality ( $p$-value for interaction $=0.040$ ) and cardiovascular mortality $(p$-value for interaction $=0.035)$. 
Table I | Baseline characteristics of statin users and non-users after propensity score matching

\begin{tabular}{|c|c|c|}
\hline & $\begin{array}{l}\text { Statin users } \\
(n=863 I)\end{array}$ & $\begin{array}{l}\text { Statin non-users } \\
\qquad(\mathrm{n}=\mathbf{8 6 3} \mathrm{I})\end{array}$ \\
\hline \multicolumn{3}{|l|}{ General characteristics } \\
\hline Age (years) & $61(11)$ & $61(13)$ \\
\hline Women (n, \%) & $4482(52)$ & $4499(52)$ \\
\hline \multicolumn{3}{|l|}{ Ethnicity (n, \%) } \\
\hline Native Dutch & $6753(78.2)$ & $684 \mid(79.3)$ \\
\hline First generation immigrant & $1297(15.0)$ & $1200(13.9)$ \\
\hline Second generation immigrant & $581(6.7)$ & $590(6.8)$ \\
\hline Annual personal income $(€)$ & $27,860[14,647-48,390]$ & $28,26 \mid[\mid 4,956-47,613]$ \\
\hline Current smoker (n, \%) & $3543(4 I .0)$ & $3540(4 I .0)$ \\
\hline \multicolumn{3}{|l|}{ I0-year cardiovascular mortality risk (SCORE) } \\
\hline $0-4 \%$ & $4757(55.1)$ & $4562(52.9)$ \\
\hline $5-9 \%$ & $2380(27.6)$ & $2175(25.2)$ \\
\hline$\geq 10 \%$ & $1493(17.3)$ & $1887(21.9)$ \\
\hline \multicolumn{3}{|l|}{ Complaints (n, \%) } \\
\hline \multicolumn{3}{|l|}{ Number of complaints } \\
\hline 0 & $345 I(40.0)$ & $3389(39.3)$ \\
\hline I & $4925(57.1)$ & $5014(58.1)$ \\
\hline 2 & $235(2.7)$ & $216(2.5)$ \\
\hline$\geq 3$ & $20(0.2)$ & $12(0.1)$ \\
\hline Chest pain & $3176(36.8)$ & $3158(36.6)$ \\
\hline Dyspnoea & $778(9.0)$ & $759(8.8)$ \\
\hline Fatigue & $277(3.2)$ & $273(3.2)$ \\
\hline Heart murmurs & $77(0.9)$ & $75(0.9)$ \\
\hline Palpitations & $1088(12.6)$ & || $4 \mid(\mid 3.2)$ \\
\hline Collapse & $63(0.7)$ & $76(0.9)$ \\
\hline \multicolumn{3}{|l|}{ Co-morbidities (n, \%) } \\
\hline Hypertension & $3386(39.2)$ & $3310(38.4)$ \\
\hline Diabetes mellitus & $843(9.8)$ & $686(7.9)$ \\
\hline Dyslipidaemia & $2078(24.1)$ & $1957(22.7)$ \\
\hline Cardiovascular diagnosis at baseline & $1061(12.3)$ & $939(10.9)$ \\
\hline \multicolumn{3}{|l|}{ Clinical characteristics } \\
\hline Body mass index $\left(\mathrm{kg} / \mathrm{m}^{2}\right)$ & $27.0(4.5)$ & $27.0(4.7)$ \\
\hline Systolic blood pressure $(\mathrm{mmHg})$ & $\mid 46(2 \mid)$ & $146(22)$ \\
\hline Total cholesterol (mmol/L) & $5.53(1.29)$ & $5.62(0.86)$ \\
\hline Low-density lipoprotein cholesterol (mmol/L) & $3.30[2.50-4.20]$ & $3.30[2.90-3.80]$ \\
\hline High-density lipoprotein cholesterol (mmol/L) & $1.40[1.10-1.80]$ & $1.40[1.10-1.80]$ \\
\hline Triglycerides (mmol/L) & $1.50[1.00-2.20]$ & $1.50[1.00-2.20]$ \\
\hline Estimated glomerular filtration rate $\left(\mathrm{mL} / \mathrm{min} / 1.73 \mathrm{~m}^{2}\right)$ & $82(27)$ & $82(28)$ \\
\hline History of cardiovascular medication use & $970(11.2)$ & $971(11.3)$ \\
\hline Cardiovascular medication prescribed at baseline & $5798(67.2)$ & $5807(67.3)$ \\
\hline
\end{tabular}




\begin{tabular}{|c|c|c|}
\hline & $\begin{array}{l}\text { Statin users } \\
(n=863 I)\end{array}$ & $\begin{array}{l}\text { Statin non-users } \\
\quad(n=863 I)\end{array}$ \\
\hline \multicolumn{3}{|l|}{ Number of cardiovascular medications } \\
\hline 0 & $2256(26.1)$ & $2240(26.0)$ \\
\hline $1-3$ & $5316(61.6)$ & $5457(63.2)$ \\
\hline $4-7$ & $1048(12.1)$ & $924(10.7)$ \\
\hline $8-10$ & II (0.I) & $10(0.1)$ \\
\hline Blood pressure lowering medication & $5388(62.4)$ & $5352(62.0)$ \\
\hline Antiplatelet or anticoagulant medication & $3086(35.8)$ & $3032(35.1)$ \\
\hline Other lipid-lowering medication & $187(2.2)$ & $191(2.2)$ \\
\hline Diabetes mellitus medication & $728(8.4)$ & $572(6.6)$ \\
\hline Nitrates & $859(10.0)$ & $803(9.3)$ \\
\hline Anti-arrhythmic medication & $108(\mathrm{I} .3)$ & $121(1.4)$ \\
\hline Digoxin & $62(0.7)$ & $65(0.8)$ \\
\hline \multicolumn{3}{|l|}{ Statin prescriptions at baseline (n, \%) } \\
\hline \multicolumn{3}{|l|}{ Statin name } \\
\hline Atorvastatin & $1859(2 \mid .5)$ & \\
\hline Fluvastatin & $42(0.5)$ & \\
\hline Pravastatin & $643(7.4)$ & \\
\hline Rosuvastatin & $889(10.3)$ & \\
\hline Simvastatin & $5198(60.2)$ & \\
\hline \multicolumn{3}{|l|}{ Statin intensity } \\
\hline Lower than guideline-recommended & $2805(32.5)$ & \\
\hline Equal to guideline-recommended & $3473(40.2)$ & \\
\hline Higher than guideline-recommended & $2353(27.3)$ & \\
\hline
\end{tabular}

Statin intensity and mortality risk

Thirty-six percent of women who were prescribed statins $(n=8 I 7 I)$ received a low-intensity statin and $26 \%$ received a high-intensity statin, compared with $29 \%$ and $28 \%$ of men who were prescribed statins $(n=8837)$, respectively. The remainder received a statin prescription at guideline-recommended dose (38\% of women vs $42 \%$ of men).

Propensity score matching resulted in a cohort of 10,688 patients ( $52 \%$ women) with a balanced confounder distribution (Supplementary Table 4). The total number of deaths was 820 in this cohort, of which 224 were cardiovascular. Higher intensity statins conferred a small protective effect against all-cause mortality $(\mathrm{HR}=0.94,95 \% \mathrm{Cl} 0.88-\mathrm{I} .00)$ and cardiovascular mortality $(\mathrm{HR}=0.86,95 \% \mathrm{Cl} 0.74-0.98)$ compared to lower intensity statins. The results were similar for women $(\mathrm{HR}=0.91,95 \% \mathrm{Cl} 0.82-\mathrm{I} .00$ and $\mathrm{HR}=0.88,95 \% \mathrm{Cl} 0.70$ I.05, respectively) and men $(\mathrm{HR}=0.96,95 \% \mathrm{Cl} 0.88-\mathrm{I} .05$ and $\mathrm{HR}=0.85,95 \% \mathrm{Cl} 0.69-\mathrm{I} .0 \mathrm{I}$, respectively) albeit not statistically significant (Table 4). There was no significant interaction between sex and statin intensity for neither all-cause mortality ( $p$-value for interaction = 0.73 ) nor cardiovascular mortality ( $p$-value for interaction $=0.76$ ). 


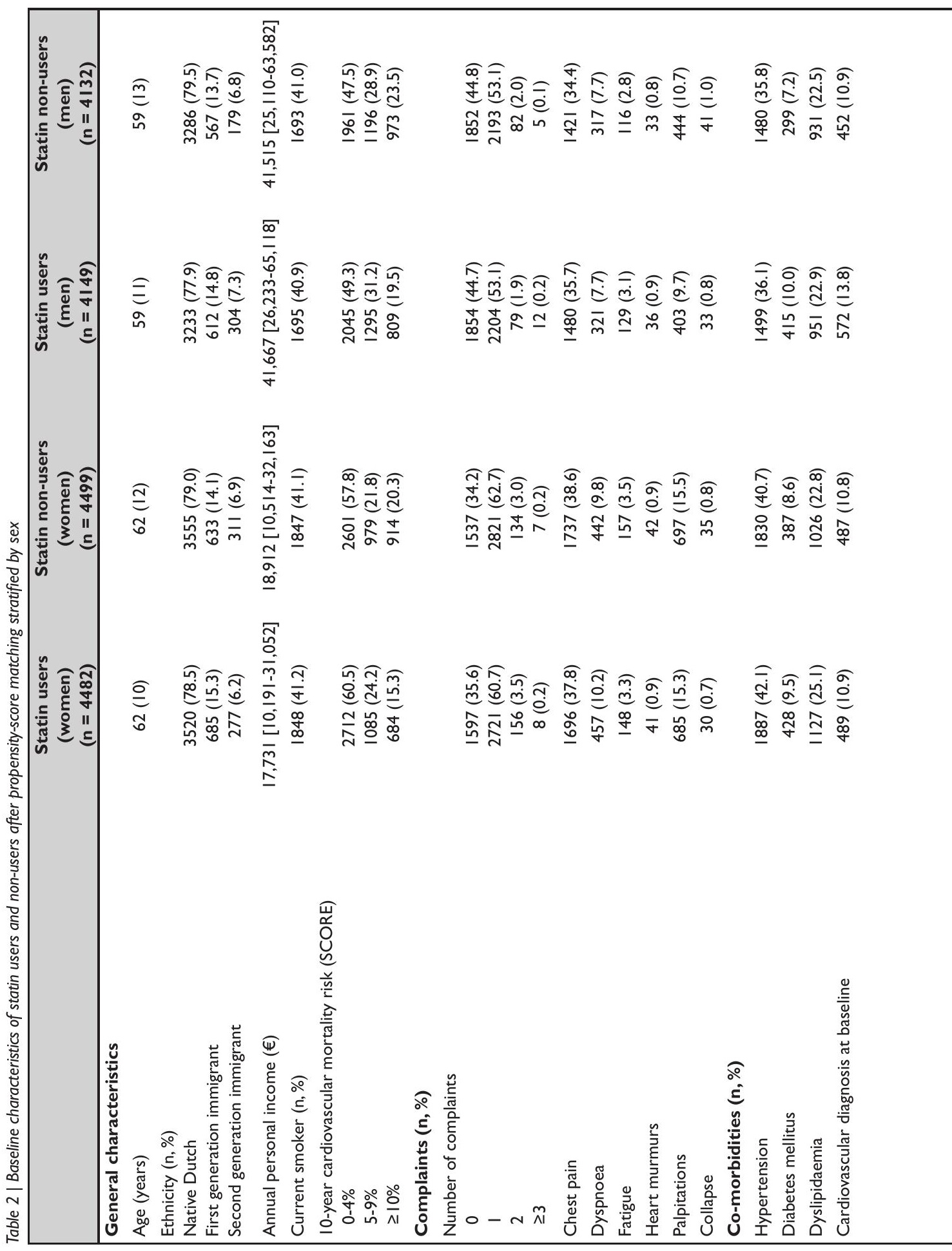




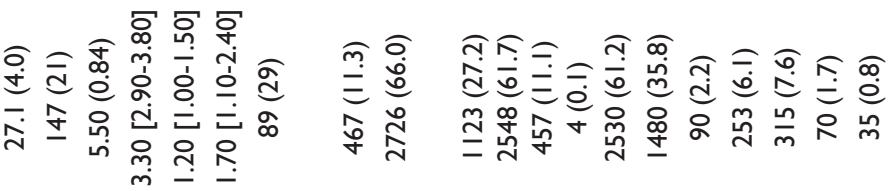

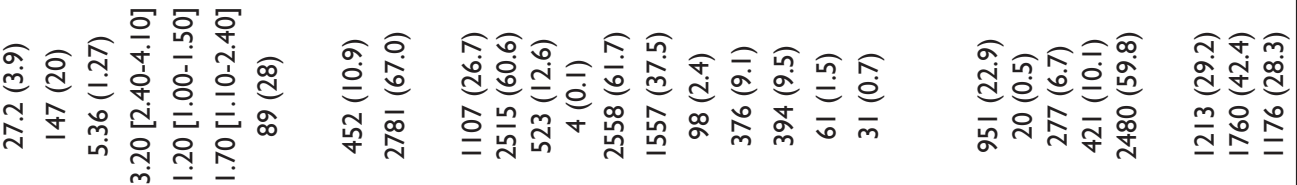

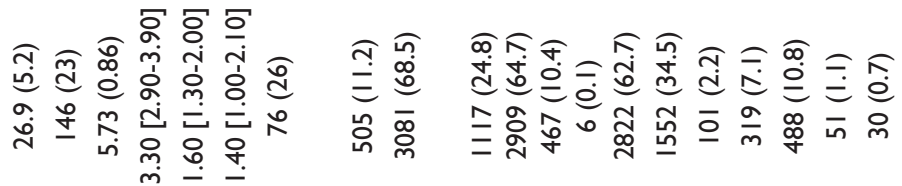

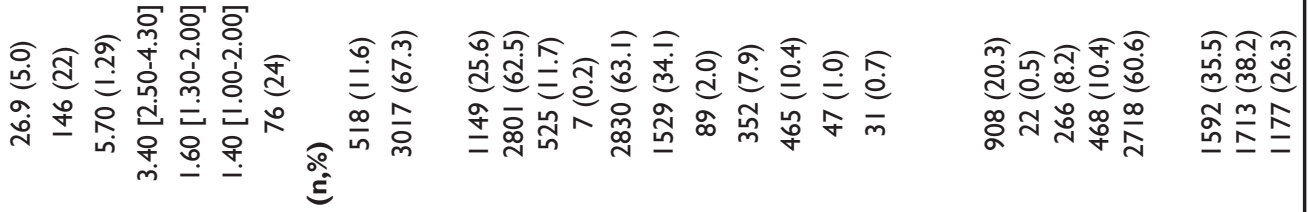

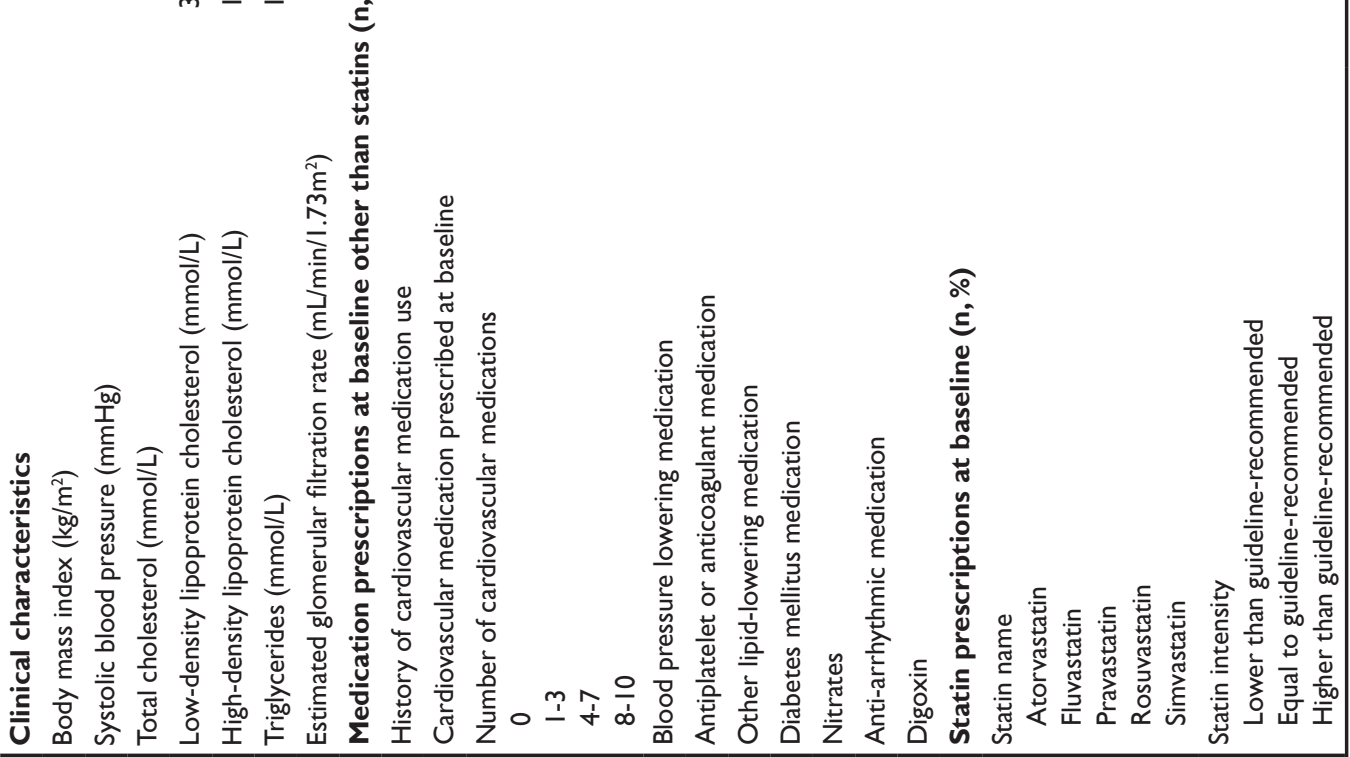


Table 3 |Adjusted hazard ratios with 95\% confidence intervals for the risk of all-cause and cardiovascular mortality associated with statin use stratified by sex

\begin{tabular}{|c|c|c|c|}
\hline & $\begin{array}{l}\text { Whole population } \\
\qquad(\mathrm{n}=\mid 7,262)\end{array}$ & $\begin{array}{l}\text { Women } \\
(n=8981)\end{array}$ & $\begin{array}{c}\text { Men } \\
(n=828 I)\end{array}$ \\
\hline \multicolumn{4}{|c|}{ All-cause mortality $\left(n_{\text {event }}=1035\right)$} \\
\hline No statin (ref) & I & I & I \\
\hline Statin & $0.76(0.7 \mid-0.82)$ & $0.66(0.58-0.74)$ & $0.89(0.81-0.96)$ \\
\hline \multicolumn{4}{|c|}{ Cardiovascular mortality $\left(\mathrm{n}_{\text {event }}=270\right)$} \\
\hline No statin (ref) & I & I & I \\
\hline Statin & $0.72(0.6 I-0.83)$ & $0.55(0.39-0.7 \mathrm{I})$ & $0.93(0.77-1.08)$ \\
\hline
\end{tabular}

Table 4 |Adjusted hazard ratios with 95\% confidence intervals for the risk of all-cause and cardiovascular mortality associated with having a statin prescription equal to or higher than guideline-recommended intensity stratified by sex

\begin{tabular}{|c|c|c|c|}
\hline & $\begin{array}{l}\text { Whole population } \\
\qquad(n=10,694)\end{array}$ & $\begin{array}{l}\text { Women } \\
(n=5598)\end{array}$ & $\begin{array}{c}\text { Men } \\
(n=5096)\end{array}$ \\
\hline \multicolumn{4}{|c|}{ All-cause mortality $\left(n_{\text {event }}=820\right)$} \\
\hline Low intensity (ref) & I & I & I \\
\hline Equal/higher intensity & $0.94(0.88-1.00)$ & $0.91(0.82-1.00)$ & $0.96(0.88-1.05)$ \\
\hline \multicolumn{4}{|c|}{ Cardiovascular mortality $\left(\mathrm{n}_{\text {event }}=224\right)$} \\
\hline Low intensity (ref) & I & I & I \\
\hline Equal/higher intensity & $0.86(0.74-0.98)$ & $0.88(0.70-1.05)$ & $0.85(0.69-1.01)$ \\
\hline
\end{tabular}

\section{Sensitivity analysis}

Repeating our analyses in those without a cardiovascular diagnosis at baseline resulted in a matched cohort of 15,180 people ( $52 \%$ women). Results for all-cause mortality were similar to the main analysis in both sexes, but the protective effect of statins for cardiovascular mortality was no longer present in men $(\mathrm{HR}=1.02,95 \% \mathrm{Cl} 0.86$ to $\mathrm{I} .18)$ when restricted to this subgroup (Supplementary Table 5). Repeating our analyses with the adapted definition of high-intensity statin did not change our results (data not shown).

\section{Discussion}

This study shows that fewer women eligible for statin therapy received statins compared with men, and when they did, they more often received a low-intensity statin. Statin prescription for primary prevention reduced the risk of all-cause and cardiovascular mortality to a greater extent in women compared with men. High-intensity statins conferred modest additional protection against both mortality outcomes and this effect was similar for women and men.

The main strength of our study was the use of a clinical care database that closely reflects the current situation in clinical care, both regarding statin prescription practices and patients that are seen at such outpatient clinics.Women were well represented $(52 \%)$ and the majority of patients $(55 \%)$ had a low ( $<5 \%)$ 10-year risk of cardiovascular mortality, which is exactly the patient population for whom more evidence regarding statin efficacy is warranted. The 
main limitation of our study is that data collection was driven by medical need and thus not performed systematically. Residual confounding may remain despite our efforts to control for confounding by indication. This may have biased our findings towards the null because patients with a higher mortality risk have a higher chance of being prescribed (high-intensity) statins, which would reduce the difference in survival between statin users and non-users and low- and high-intensity statin users. We were also unable to evaluate more short-term outcomes that may be relevant in this low-risk population such as cardiovascular events and hospitalisations, and lacked good quality data on adverse drug reactions. We were unable to correct for potential sex differences in adherence, but this is unlikely to change the interpretation of our findings because literature suggests women have poorer adherence than men, which would lead us to underestimate the observed benefit in women ${ }^{3,18}$.

Our finding that the protective effect of statins was stronger in women compared with men corresponds to findings from previous studies looking at sex differences in statin effectiveness ${ }^{2,10-12}$. The protective effect of statins was not seen in men after the exclusion of those diagnosed with cardiovascular disease at baseline. This is in line with findings from a meta-analysis that showed statin use for primary prevention reduced mortality in women but not men, while statin use for secondary prevention did reduce mortality in men ${ }^{2}$. Literature also suggests low-risk patients could gain most benefit from statin treatment ${ }^{2,19}$. This may explain both why we found such a strong effect of statin treatment in our whole cohort compared with previous trials, and why we found a stronger effect in women compared with men. Our study population had a low median 10-year cardiovascular mortality risk of approximately $3.5 \%$ percent. Women had a lower average mortality risk than men $(2.9 \%$ versus $4.1 \%$, respectively), and fewer women in both the statin-treated and statin-untreated group had a high mortality risk ( $\geq 10 \%)$ compared with men. Another potential explanation might be that women adhered better to lifestyle recommendations such as dietary advice provided complementary to statin treatment than men, but literature on this topic is scarce ${ }^{20}$. In addition, our findings need to be validated. This may be difficult within a trial setting due to the long follow-up time required to accrue sufficient mortality endpoints in these relatively healthy populations. Other observational studies using real-world data may offer valuable insights, provided they are able to properly correct for indication bias. Such studies can also evaluate the generalisability of our findings. Our database only includes Dutch patients located at the unique intersection between primary and secondary care, and we had to exclude the healthiest and least healthy patients because these could not be matched to a counterpart with a sufficiently similar propensity score. It remains unclear how our findings translate to these patient groups.

Women in our dataset more often received low-intensity statins compared with men, confirming previous work ${ }^{3-5}$. The evidence supporting equal statin prescription strategies for both sexes is growing, but higher rates of side effects in women may be the underlying reason for maintaining this careful approach to statin prescription in women. The topic of statin- 
related side effects is still debated. Meta-analyses suggesting no differences in adverse events

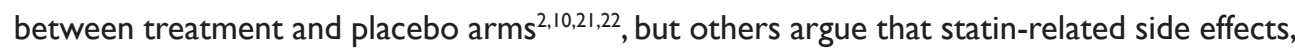
especially milder ones, are very common and an important challenge in statin treatment ${ }^{23}$. There is also some evidence for sex differences in adverse events ${ }^{2,3,21}$, but this is not yet conclusive. Our analyses exploring the relationship between statin intensity and long-term prognosis suggested high-intensity regimens only conferred marginal additional benefit. This raises the question whether low-intensity statins could already be sufficiently effective in women, and whether the small additional benefit of high-intensity statins is proportional to the increased risk of potential side effects. These yet unanswered questions underscore the importance of clinical trials providing sex-specific data for both the efficacy of low- versus high-intensity statins and adverse events rates associated with different statin intensities. Dose-finding trials could answer the question which statin dosage best balances prognosis benefits against possible harms such as side effects.

\section{Conclusion}

Fewer women received statins than men, and when they did, they were more likely to receive low-intensity regimens. The protective effect of primary prevention statins was stronger in women than men for both all-cause and cardiovascular mortality, but the additional benefit of high-intensity statins was modest and similar for both sexes. Statins seem to be effective regardless of treatment intensity, especially in women.

\section{Acknowledgements}

Results are based on calculations by the authors using non-public microdata from Statistics Netherlands.

\section{Funding}

This work was supported by the Dutch Cardiovascular Alliance consortium DCVA IMPRESS (2020B004) and ERC Consolidator grant UCARE (866478).

\section{Conflict of interest}

LH, IT and GS are employed by Cardiology Centers of the Netherlands. All other authors declare no competing interests. 


\section{References}

I. Cholesterol Treatment Trialists Collaboration. Efficacy and safety of more intensive lowering of LDL cholesterol: a meta-analysis of data from 170000 participants in 26 randomised trials. The Lancet 2010; 376(9753): 1670-8I.

2. Kostis WJ, Cheng JQ, Dobrzynski JM, Cabrera J, Kostis JB. Meta-Analysis of Statin Effects in Women Versus Men. Journal of the American College of Cardiology 2012; 59(6): 572-82.

3. Nanna MG, Wang TY, Xiang Q, et al. Sex Differences in the Use of Statins in Community Practice. Circulation: Cardiovascular Quality and Outcomes 2019; I2(8): e005562.

4. Peters SAE, Colantonio LD, Zhao H, et al. Sex Differences in High-Intensity Statin Use Following Myocardial Infarction in the United States. Journal of the American College of Cardiology 20I8; 7I (I6): I729-37.

5. Virani SS, Woodard LD, Ramsey DJ, et al. Gender Disparities in Evidence-Based Statin Therapy in Patients With Cardiovascular Disease. American Journal of Cardiology 20I5; II5(I):21-6.

6. Olmastroni E, Boccalari MT,Tragni E, et al. Sex-differences in factors and outcomes associated with adherence to statin therapy in primary care: need for customisation strategies. Pharmacological Research 2019: I045I4.

7. Cangemi R, Romiti GF, Campolongo G, et al. Gender related differences in treatment and response to statins in primary and secondary cardiovascular prevention: The never-ending debate. Pharmacological Research 2017; II7: 148-55.

8. Reiner Ž. Statins in the primary prevention of cardiovascular disease. Nature Reviews Cardiology 20I3; I0(8):453-64.

9. Fulcher J, O'Connell R,Voysey M, et al. Efficacy and safety of LDL-lowering therapy among men and women: metaanalysis of individual data from I74,000 participants in 27 randomised trials. Lancet 20I5; 385(9976): I397-405.

10. Samia Mora, Robert J. Glynn, Judith Hsia, Jean G. MacFadyen, Jacques Genest, Ridker PM. Statins for the Primary Prevention of Cardiovascular Events in Women With Elevated High-Sensitivity C-Reactive Protein or Dyslipidemia. Circulation 2010; 121 (9): 1069-77.

II. Ray KK, Seshasai SRK, Erqou S, et al. Statins and All-Cause Mortality in High-Risk Primary Prevention:A Metaanalysis of II Randomized Controlled Trials Involving 65229 Participants. Archives of Internal Medicine 20I0; I70(I2): I024-3I.

12. Walsh JME, Pignone M. Drug Treatment of Hyperlipidemia in Women. JAMA 2004; 29I(I8): 2243-52.

13. Banga J, Van Dijk J, Van Dis I, Giepmans L, Goudswaard A, Grobbee D. NHG-Standaard Cardiovasculair risicomanagement (tweede herziening). Huisarts Wet 20।2; 55: I4-28.

14. Burgers J, Simoons M, Hoes A, Stehouwer C, Stalman W. Guideline'Cardiovascular Risk Management'. Nederlands tijdschrift voor geneeskunde 2007; I5I(I9): I068-74.

15. Law MR,Wald NJ, Rudnicka AR. Quantifying effect of statins on low density lipoprotein cholesterol, ischaemic heart disease, and stroke: systematic review and meta-analysis. BMJ 2003; 326(7404): I423.

16. van Buuren S, Groothuis-Oudshoorn K. mice:Multivariate Imputation by Chained Equations in R. 20 I I 20 I I;45(3):67.

17. Ho D, Imai K, King G, Stuart EA. Matchlt: Nonparametric Preprocessing for Parametric Causal Inference. 20I I 20I I;42(8): 28.

18. Bots SH, Inia JA, Peters SAE. Medication Adherence After Acute Coronary Syndrome in Women Compared With Men:A Systematic Review and Meta-Analysis. Frontiers in GlobalWomen's Health 202I;2(I).

19. Cholesterol Treatment Trialists Collaboration, Mihaylova B, Emberson J, et al.The effects of lowering LDL cholesterol with statin therapy in people at low risk of vascular disease: meta-analysis of individual data from 27 randomised trials. Lancet 2012;380(984I): 58I-90.

20. Szymczyk I,Wojtyna E, LukasW,Kępa J, Pawlikowska T.How does gender influence the recognition of cardiovascular risk and adherence to self-care recommendations?: a study in polish primary care. BMC Family Practice 2013 ; I4(I): 165.

2I. Hsue PY, Bittner VA, Betteridge J, et al. Impact of Female Sex on Lipid Lowering, Clinical Outcomes, and Adverse Effects in Atorvastatin Trials. The American Journal of Cardiology 20 I 5; I I 5(4):447-53.

22. Taylor F, Huffman MD, Macedo AF, et al. Statins for the primary prevention of cardiovascular disease. Cochrane Database Syst Rev 2013; 2013(I): CD0048I6-CD.

23. Redberg RF, Katz MH. Statins for Primary Prevention:The Debate Is Intense, but the Data Are Weak. JAMA Internal Medicine 20I7; I77(I):2I-3. 


\section{Supplementary files}

\section{Supplementary tables}

Supplementary Table I | Classification of statin regimens as lower, equal or higher intensity depending on how much they lower low-density lipoprotein cholesterol levels compared to the Dutch guideline recommended $40 \mathrm{mg}$ simvastatin (37\% lowering)

\begin{tabular}{|lll|}
\hline Lower intensity & Equal intensity & Higher intensity \\
\hline Atorvastatin $5 \mathrm{mg}$ & Atorvastatin $10 \mathrm{mg}$ & Atorvastatin $>10 \mathrm{mg}$ \\
Fluvastatin any dose & & Rosuvastatin $>2.5 \mathrm{mg}$ \\
Pravastatin any dose & & Simvastatin $>40 \mathrm{mg}$ \\
Rosuvastatin $2.5 \mathrm{mg}$ & & \\
Simvastatin $<40 \mathrm{mg}$ & & \\
\hline
\end{tabular}


Supplementary Table 2 | List of variables included in the propensity score

\begin{tabular}{|c|c|}
\hline Variable & Additional sub-classifications \\
\hline \multicolumn{2}{|l|}{ Sex } \\
\hline \multicolumn{2}{|l|}{ Age } \\
\hline \multirow[t]{5}{*}{ Cardiovascular diagnosis at baseline } & Heart failure \\
\hline & Coronary heart disease \\
\hline & Cardiovascular intervention \\
\hline & Cerebrovascular \\
\hline & Congenital \\
\hline \multicolumn{2}{|l|}{ Ethnicity } \\
\hline \multicolumn{2}{|l|}{ Personal income } \\
\hline \multicolumn{2}{|l|}{ Height } \\
\hline \multicolumn{2}{|l|}{ Weight } \\
\hline \multicolumn{2}{|l|}{ Body mass index } \\
\hline \multicolumn{2}{|l|}{ Systolic blood pressure } \\
\hline \multicolumn{2}{|l|}{ Diastolic blood pressure } \\
\hline \multirow[t]{6}{*}{ Complaints at baseline } & Chest pain \\
\hline & Dyspnoea \\
\hline & Fatigue \\
\hline & Heart murmur \\
\hline & Palpitations \\
\hline & Collapse \\
\hline \multicolumn{2}{|l|}{ Number of complaints at baseline } \\
\hline \multirow[t]{3}{*}{ Comorbidities at baseline } & Diabetes mellitus \\
\hline & Hypertension \\
\hline & Dyslipidaemia \\
\hline \multicolumn{2}{|l|}{ Current smoker } \\
\hline \multicolumn{2}{|l|}{ Total cholesterol } \\
\hline \multicolumn{2}{|l|}{ High-density lipoprotein cholesterol } \\
\hline \multicolumn{2}{|l|}{ Low-density lipoprotein cholesterol } \\
\hline \multicolumn{2}{|l|}{ Creatinine } \\
\hline \multicolumn{2}{|l|}{ Estimated glomular filtration rate } \\
\hline \multicolumn{2}{|l|}{ Triglycerides } \\
\hline \multicolumn{2}{|l|}{ History of cardiovascular medication } \\
\hline \multirow[t]{7}{*}{ Cardiovascular medication prescribed at baseline } & Blood-pressure lowering \\
\hline & Antiplatelet or anticoagulation \\
\hline & Lipid-lowering other than statins \\
\hline & Diabetes mellitus \\
\hline & Nitrates \\
\hline & Anti-arrhythmic \\
\hline & Digoxin \\
\hline \multicolumn{2}{|l|}{ Number of medication prescriptions } \\
\hline Baseline appointment after 2016 & \\
\hline
\end{tabular}




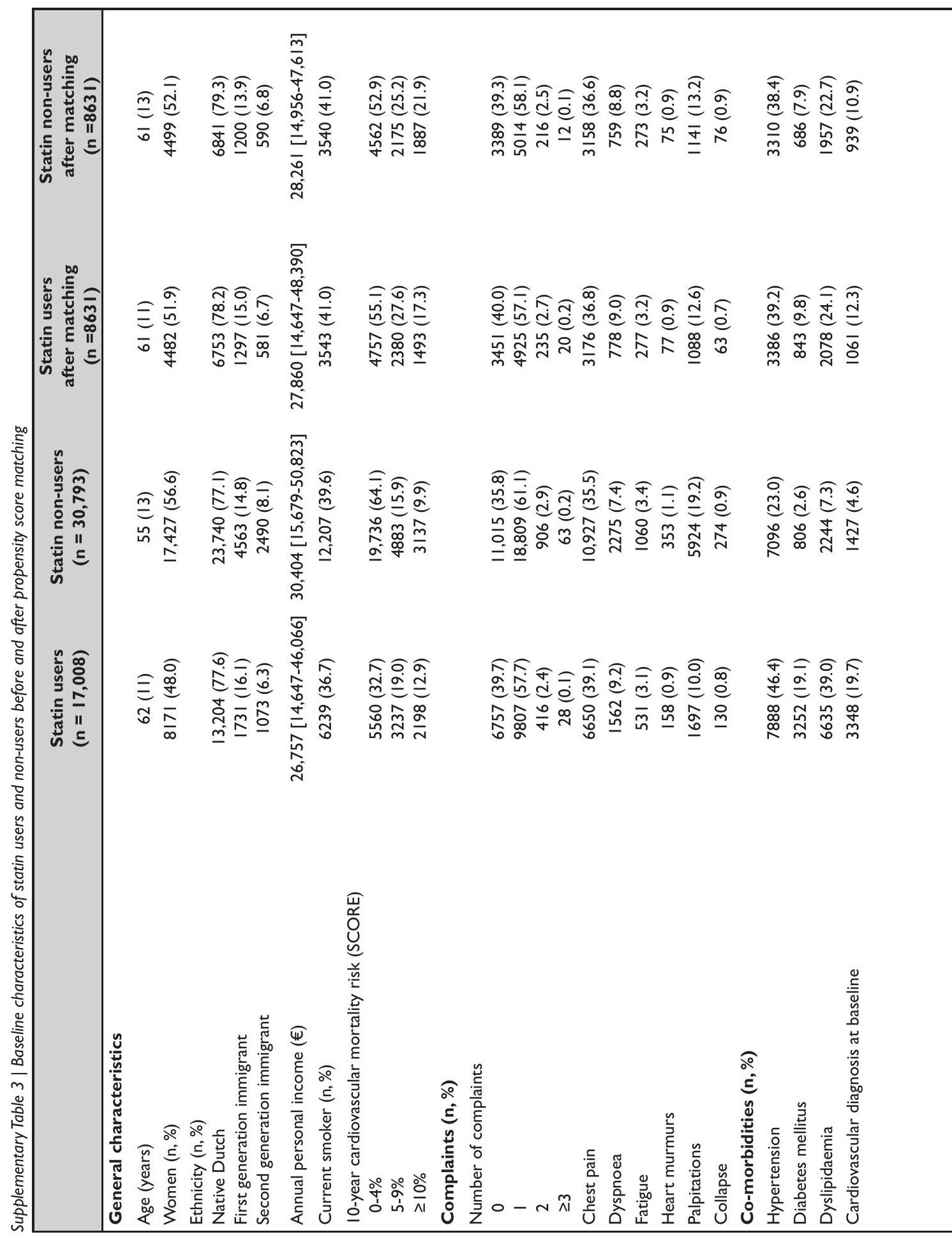




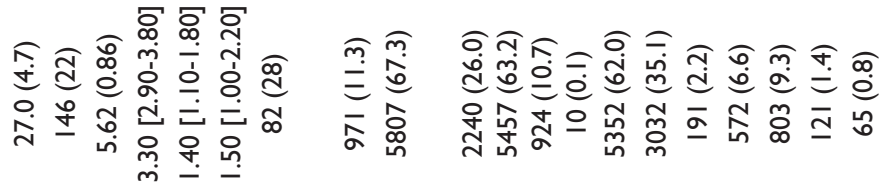

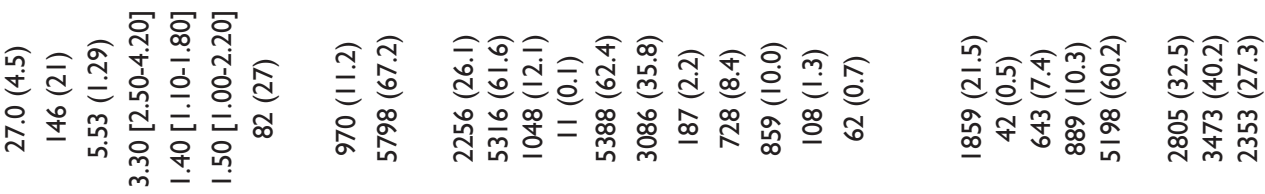

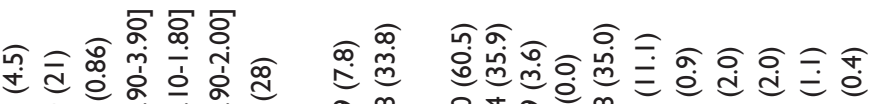

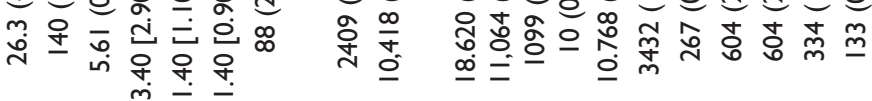

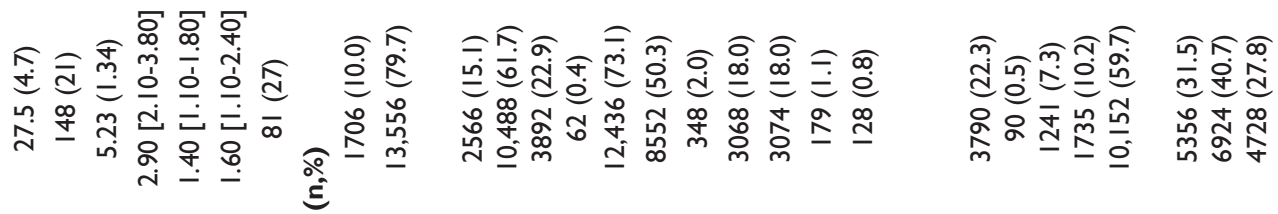

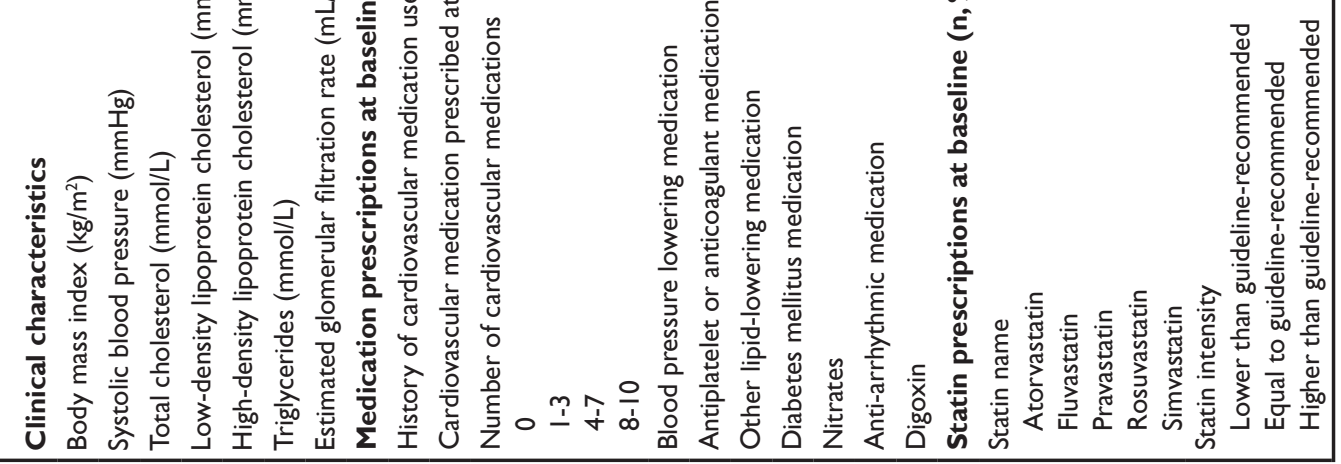




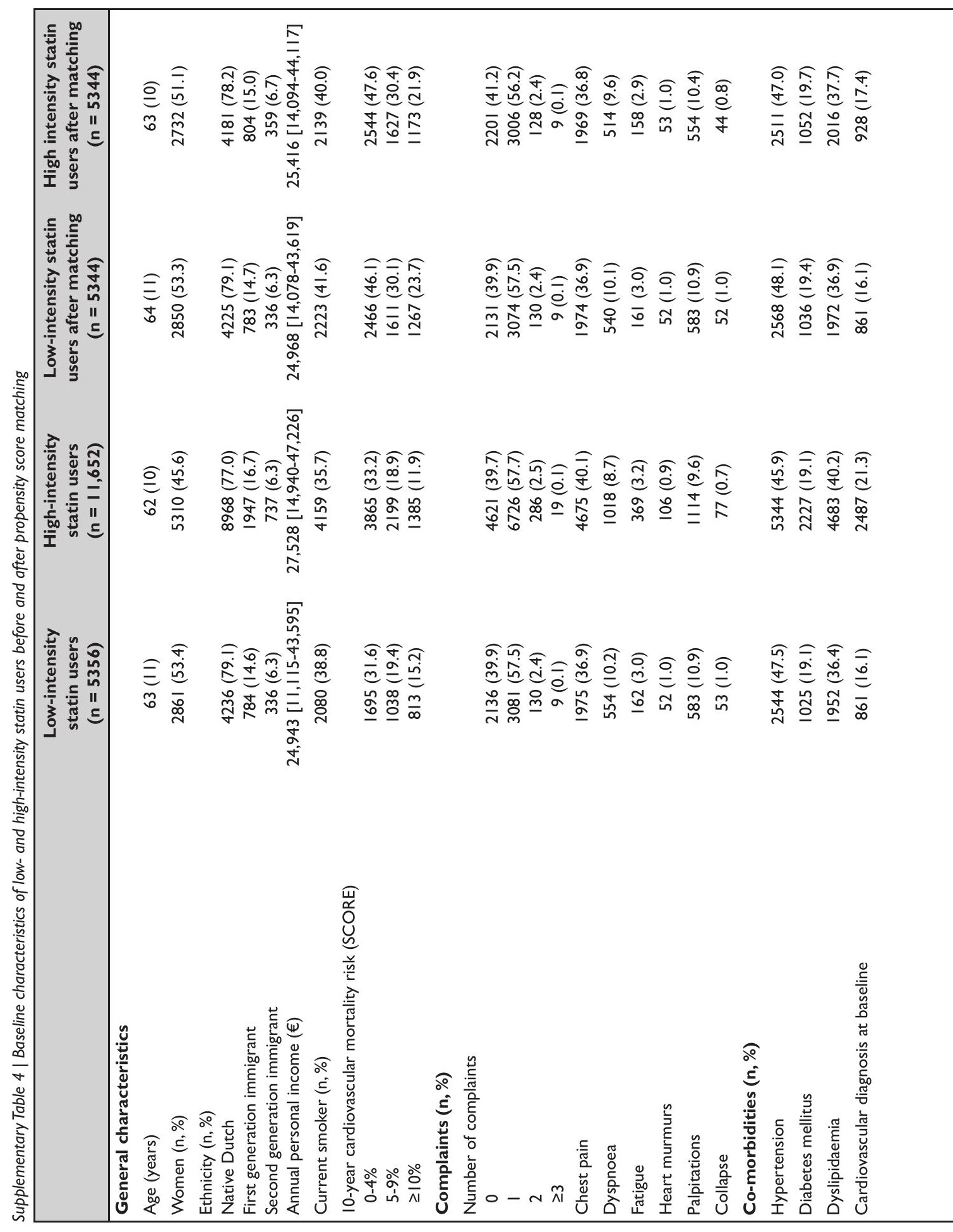




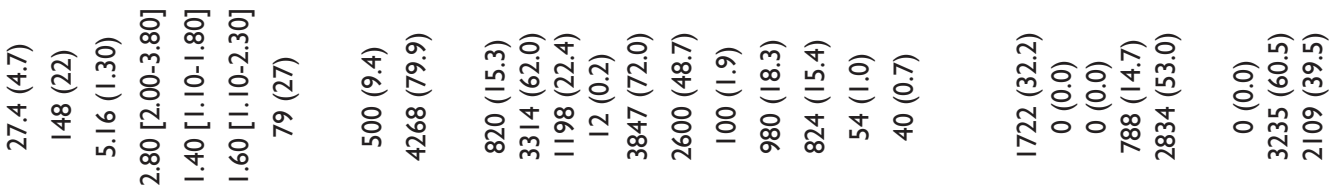

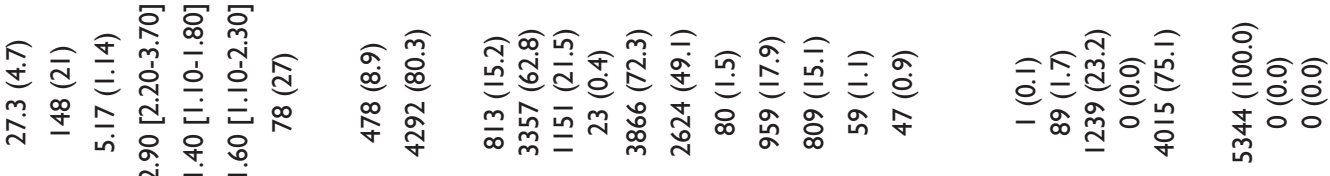

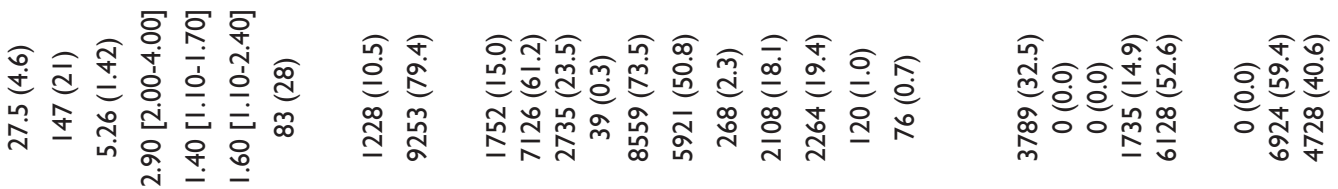

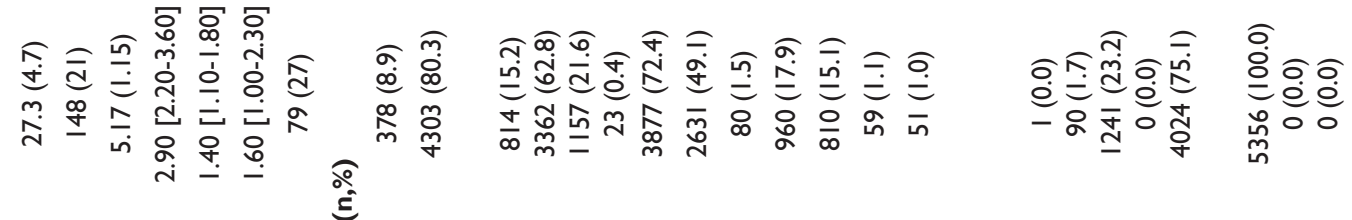

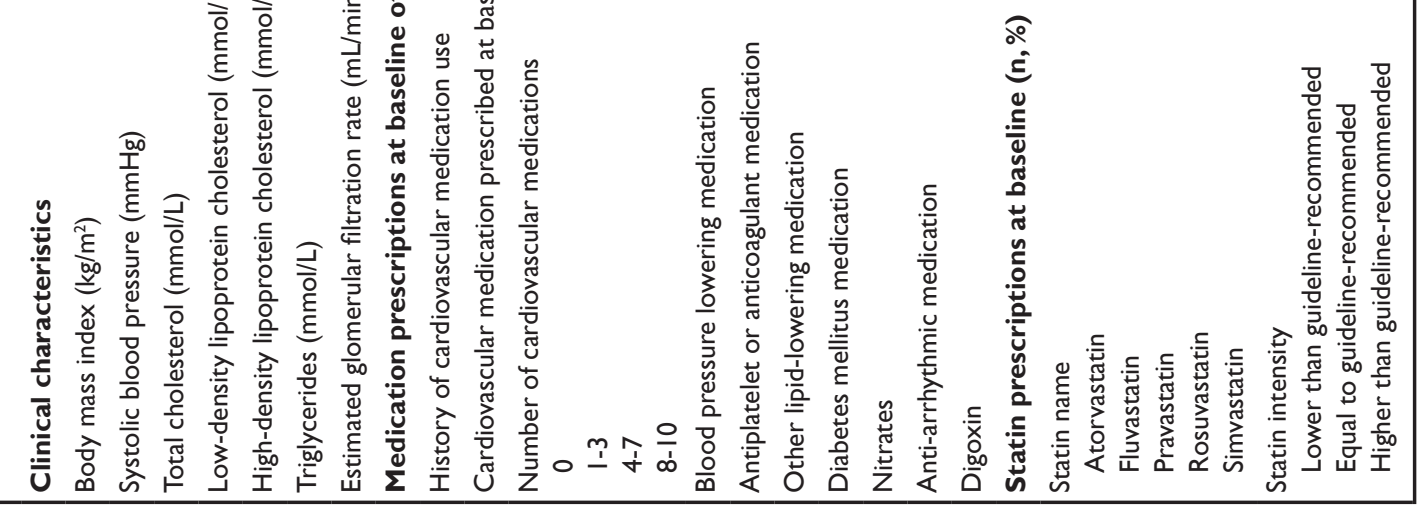


Supplementary Table 5 | Adjusted hazard ratios for the risk of all-cause and cardiovascular mortality associated with statin use stratified by sex in those without a cardiovascular diagnosis at baseline

\begin{tabular}{|lccc|}
\hline & $\begin{array}{c}\text { Whole population } \\
(\mathbf{n = 1 5 , 1 8 0 )}\end{array}$ & $\begin{array}{c}\text { Women } \\
(\mathbf{n = 7 9 3 0})\end{array}$ & $\begin{array}{c}\text { Men } \\
(\mathbf{n}=\mathbf{7 2 5 0})\end{array}$ \\
\hline $\begin{array}{l}\text { All-cause mortality } \\
\text { No statin (ref) }\end{array}$ & $\mathrm{I}$ & $\mathrm{I}$ & $\mathrm{I}$ \\
$\begin{array}{l}\text { Statin } \\
\text { Cardiovascular mortality } \\
\text { No statin (ref) }\end{array}$ & $0.78(0.72-0.84)$ & $0.68(0.60-0.77)$ & $0.89(0.81-0.97)$ \\
Statin & $\mathrm{I}$ & $\mathrm{I}$ & $\mathrm{I}$ \\
\hline
\end{tabular}


STATINS REDUCE MORTALITY MORE STRONGLY IN WOMEN 

General discussion

8 


\section{General discussion}

\section{Short summary}

It has been suggested that biological differences between women and men affect both the efficacy and the safety of cardiovascular medication ${ }^{1-5}$, but translating this knowledge to clinical care is hampered by a lack of sex-specific data. This is especially relevant for the syndrome of heart failure (HF), which presents a growing healthcare concern and manifests differently in women compared with men $^{6}$. Sex-specific data on the optimal dosing of and adverse drug reactions (ADRs) associated with HF medication is needed to fuel the discussion about tailoring HF treatment strategies to patient sex to improve clinical care. Chapter $\mathbf{2}$ and Chapter $\mathbf{3}$ demonstrated that this information is unlikely to be found within published literature, identifying a pressing need for datasets with proper representation of women to address these questions. Clinical care datasets such as the one presented in Chapter $\mathbf{4}$ would fit this bill nicely because of their unique broad scope across patient groups and clinical measures. This thesis therefore studied the potential of routine clinical care data for addressing and closing existing evidence gaps concerning sex-specific dosage of HF medication and sex-specific ADR risk in HF patients. Chapter $\mathbf{5}$ showed that women more often discontinue cardiovascular medication than men because of ADRs even after correction for prescription differences, but also demonstrated quality issues regarding reporting and recording of ADRs in clinical care datasets. Chapter 6 suggested women with HFrEF may do better on lower medication dosages than those currently recommended in the guidelines, but the relatively small number of HF patients included complicated drawing sound conclusions, partially because the statistical techniques applied to account for indication bias are less reliable in small datasets. Chapter $\mathbf{7}$ found that women benefitted from statin treatment regardless of dosage and suggested that lower dosages might be sufficient in women, but the interpretation of these findings were complicated by the fact that we were unable to explain why the observed effect sizes were much larger than expected based on previous work.

These last three chapters show that clinical care data are a valuable resource for expanding the available sex-specific data. However, while each chapter successfully addressed an existing evidence gap, it proved difficult to fully close each gap based on clinical care data alone due to the inherent limitations of these datasets. This chapter first discusses short- and longterm strategies to reduce the effect of these limitations and how these could increase the potential of clinical care datasets. It then puts this thesis in a broader perspective, discussing whether clinical care data alone will be sufficient to close the evidence gaps addressed in this thesis or a more comprehensive approach is needed. 


\section{Generating evidence where there is none: the potential of clinical care datasets}

The main attraction of clinical care datasets is their potential to generate evidence in a relatively short time period, without the need for laborious and expensive data collection associated with clinical trials and cohort studies. However, clinical care data also come with their unique quirks that set them apart from data collected for research purposes ${ }^{7-11}$. The most important difference is the reason behind data collection. Clinical care data is collected primarily for the purpose of delivering clinical care9, meaning data collection is driven by medical need.This is inherently different from research datasets, where data is collected with the purpose of answering specific research questions. The next two sections will discuss how this affects key aspects of the collected data. The first section will introduce the theoretical framework for research with clinical datasets, and the second section will translate that theory into practice using examples from this thesis.

\section{The theory behind clinical care data-based research}

Having medical need as the driver for data collection has various effects on the data, which can be divided into the comprehensiveness and the quality of the data ${ }^{9}$. Comprehensiveness describes whether the dataset contains all relevant information about a single patient ${ }^{9}$. Often clinical care datasets only capture a snapshot of the patient that contains the information clinically relevant at that time and thus do not tell a complete story. Information may remain out of reach, either because clinical care centres cannot collect information on patients that never visit or those that leave after being treated, or because one part of the system (hospitals) cannot access other parts of the system (general practitioners), or because there is no medical reason to collect certain information. It may also be impossible to record certain information, for example due to time constraints or because equipment is not available, or because certain conditions do not yet exist as a designated diagnosis term or code $^{7,10,11}$.

Quality is about how well the collected information reflects the truth about a single patient. Data entry in clinical care datasets is performed by healthcare professionals for healthcare professionals and is not subjected to extensive quality control. Entered information may be incorrect, inaccurate, incomplete, or incomprehensible ${ }^{10,11}$. These quality issues can arise both unintentionally (randomly) and systematically. Examples of the former are typos or something not being entered by accident. Examples of the latter are reimbursement coding systems that tend to mislabel certain conditions, diagnostic codes that change over time $^{10,11}$, or the use of ambiguous abbreviations in free text summaries. Another important aspect of quality is whether the recorded information can be operationalised. For example, information recorded in free text format cannot be used without properly developed text retrieval methods ${ }^{10}$. 
These considerations lie at the basis of deciding whether a clinical care dataset is fit for purpose, meaning that the information collected is of sufficient comprehensiveness and quality to answer the research question posed ${ }^{7}$. Another concept that ties in with this is validity, which is about whether the size of random and systematic error interferes with drawing sound conclusions ${ }^{8}$. Taken together, these concepts offer researchers a crude guide for deciding whether or not to use clinical care data. However, this crude guide is not always sufficient to navigate the many nuances and grey areas surrounding the use of clinical care data. Often researchers have to balance the limitations of a clinical care dataset against the opportunities, for example in some areas of sex differences research where the only data available comes from clinical care datasets. Some authors have published examples of how to deal with these kind of trade-offs ${ }^{12-14}$, but literature on this topic is scarce. The next section will translate the theory into practice using examples from this thesis, with the aim of providing a practical guide to performing research with clinical care data.

\section{The reality of working with existing clinical care databases}

\section{(1) Not everything is black or white: performing a realistic fitness for purpose check}

The first step of any project using clinical care data is the fitness for purpose check as described in the previous section. In practical terms, this entails defining the study population (domain), exposure of interest (determinant), endpoint of interest (outcome) and any other relevant clinical parameters (confounders and effect modifiers) needed to answer the research question. When the study population fits the domain and the determinants, outcomes and covariables are present and properly recorded, the dataset is fit for purpose.

However, fitness for purpose also depends on factors outside of the dataset. In the case of sex differences research, the scarcity of available data and need for answers can balance out against the dataset's inherent limitations. Chapters 5 and 6 are good examples of this. The outcome variable of Chapter 5, ADRs, was poorly recorded in our clinical care dataset compared with for example pharmacovigilance databases or clinical trials. Pharmacovigilance databases have a highly structured approach to ADR categorisation, resulting in data that are easy to analyse. Clinical trials are obligated to record all adverse events experienced by participants, resulting in good quality follow-up that catches all potential ADRs. Our clinical care dataset had neither of these things, recording ADRs as free text and relying on patient and physician self-report to catch events. However, it also had its own advantages. Pharmacovigilance datasets lack data on prescription and thus cannot correct for sex-specific prescription practices, which we could. Clinical trials are limited in the number and type of ADRs they can pick up because they often include specific subpopulations that are followed up for a relatively short time period, whereas our dataset comprised data from patients across the medical spectrum collected over a 10 year period. 
In Chapter 6 the patient selection was challenging because the diagnosis of heart failure (HF) itself, and especially its subtypes, is complex and requires multiple clinical parameters ${ }^{15}$ that are not always part of the standard clinical workup. Research studies work around this by either limiting recruiting sites to specific locations that do measure these parameters or performing these measurements themselves as part of the recruitment process. While this approach may result in highly detailed phenotyping of the HF patients included in such a study ${ }^{16}$, it ignores all HF patients that will never be subjected to such detailed phenotyping because they are for example treated by their general practitioner who does not have the resources to do it. In these patients, ejection fraction (EF) is an important clinical parameter in deciding HF phenotype. Our dataset addresses exactly this patient population and had sufficient information available on EF to allow for clinically meaningful subgroups, turning the unique position between the general practitioner and the hospital into a strength instead of a limitation.

Both of these chapters are good examples of situations where the need for answers can balance out against limitations of the dataset. This allows researchers to search for the best possible alternative that will enable them to answer their research question in a reliable way. The decision whether the relevance of a project outweighs the dataset limitations differs per project and situation. If for Chapter 6 ejection fraction data had not been available, this limitation could have outweighed the clinical relevance leading to cancellation of the project. Of course, choosing the best possible alternative will always be connected to inherent limitations. However, being hung up on those limitations and therefore abandoning all projects that cannot be performed under perfect conditions is unrealistic and undermines the potential advantages clinical care data can bring. The fitness for purpose check, while being a 'black-or-white' decision moment, should thus incorporate all these grey area considerations that balance relevance and advantages against limitations.

\section{(2) Tell me who you (do not) see: the risk of introducing selection bias}

Clinical care datasets are comprised of a heterogeneous population, meaning that often not all patients in the dataset belong to the relevant domain for a specific research question. This is true for all three analysis chapters in this thesis.

Even though selecting a subset of patients does not necessarily leads to selection bias, exclusion criteria should be considered carefully because in clinical care datasets absence of information is not always equal to absence of a specific criterion. For example, in Chapter 6 there were several ways in which we could have defined and selected patients with HF from our dataset. We could have used an available diagnostic score or made our own based on a combination of echocardiographic and laboratory parameters. Naturally, those without information on these parameters would have to be excluded. As mentioned earlier, existing diagnostic scores or parameter cut-off strategies for HF often rely on highly 
specific biomarkers that are not routinely measured in clinical care. This selection strategy will thus favour HF patients who undergo additional workup for these specific parameters, which are probably the more severe cases, while the original domain of the study was all HF patients. This introduces selection bias if the effect of medication on mortality differs between patients with mild and severe HF. We therefore considered selecting patients based on having a diagnosis of HF in their medical record the safer choice, because the medical record is collected and updated similarly for all patients.

Selection (bias) can also occur in other stages of a research project. In Chapter 7, a large number of both statin users and statin non-users were excluded because they could not be matched to a sufficiently similar counterpart based on their propensity score. The excluded statin users were less healthy compared to the included users, given they had more comorbidities and concomitant medication prescriptions. The excluded non-users, on the other hand, were younger and healthier compared to the included non-users. For the latter one could argue that they should have been excluded in the first place because they were highly unlikely to ever receive a statin based on Dutch guidelines and thus did not fit the domain of the study, but the fact remains that Chapter 7 excluded the more extreme patients on both sides of the spectrum. However, propensity score matching was one of the preferred methods to deal with confounding by indication for these types of analysis. We therefore chose to stick with our method because it was the most reliable option.

There can also be selection at the gate and selection at the exit, both of which are common in clinical care datasets. Selection at the gate occurs when not all patients from the domain of interest are equally likely to enter a clinical dataset, whereas selection at the exit occurs when not all patients from the domain of interest are equally like to be followed up over time. The latter can in some cases be circumvented through passive follow-up via record linkage, which was used in Chapter 6 and 7. The former is difficult to remediate, but the degree of selection can be tested by comparing the study population against a random sample of the general population as provided in Chapter 4.

Considering which patients are (not) seen in a particular clinical care dataset and whether this selection may introduce bias helps to separate clinically relevant questions that can be answered with clinical care data from those that cannot. This not only highlights the potential of clinical care datasets, but also identifies which clinically relevant questions still require a cohort study or clinical trial to be answered and thus should have priority on the research agenda.

\section{(3) There is a reason for that: mechanisms underlying missing data}

The next step is checking clinical care data for missing values and choosing the best approach to deal with them. Imputation of missing data, through simpler single imputation methods or through multiple imputation, has become a methodological mainstay in epidemiological research. It has been incorporated into statistical programmes such as $\mathrm{R}^{17}$, making it both widely available and easily accessible. However, it is important to realise these methods 
were developed based on research data and that they make implicit assumptions about why missing values occur. Specifically, they assume that the chance of something being missing is not related to unobserved covariates. Or in other words, that the data collector tried to collect each piece of information with equal effort and thus missing values occur either by chance (they forgot to enter it; missing completely at random) or can be predicted based on the information that was collected (older people were less likely to show up; missing at random). However, this assumption may not always hold for clinical care data.

First because there is a type of missing data unique to clinical care data called informative missingness. This occurs when the absence of information in itself has value and does not call for imputation but rather interpretation of what the absence means. For example, when a medication end date is missing the patient is unlikely to have experienced an ADR (Chapter 5), when a diagnosis of HF is missing a patient does not have HF (Chapter 6), or when a statin prescription is missing the patient is not a statin user (Chapter 7). These assumptions may be specific to a particular clinical care dataset or may hold true across datasets and must always be made explicit for the sake of transparency. Second, there are missing values whose occurrence is driven by unmeasured or unknown variables (missing not at random). For example, severely ill HF patients with a high mortality risk are less likely to have their ejection fraction measured at CCN because they are immediately referred to a hospital. This type of missingness is not unique to clinical care datasets and impossible to prove, which means that researchers often assume data is missing at random and thus can be safely imputed, similar to Chapters 6 and 7. Using missing data strategies that assume data is missing completely at random, such as complete case analysis, is discouraged because there often is a reason why missing data occurs in clinical care databases. As statistical methods that deal with missing not at random are not yet available, assuming that data is missing at random and subsequently using multiple imputation to deal with missing values is probably the best approach at the moment.

\section{(4) On correlation and causation: approaches to causal inference}

Statistical analysis and interpretation of results are the last important steps of research projects. As clinical care data are observational, any suggestions about causality should be considered very carefully in light of all potential biases and unmeasured confounders. Causal inference, the process of judging whether a relationship may be causal, is a staple of epidemiological research ${ }^{18,19}$. Over time, this process gradually focussed on statistical or mathematical tools that reduce the effect of confounders as much as possible to approach a randomised trial setting ${ }^{18}$. The propensity score methods used in Chapter 6 and Chapter 7 are a good example of this. These methods were developed to deal with confounding by indication, a type of confounding where treatment assignment is not random but dependent on the characteristics of the patient. Propensity score methods can rebalance the likelihood of treatment assignment either through matching or weighting, resulting in a randomised 
trial-like situation where confounders are relatively equally balanced between treatment groups $^{20}$. This ties in with the target trial concept from the counterfactual framework ${ }^{21}$, which suggests researchers design a fictional target trial that answers their research question and then check how well their observational dataset can emulate this target trial. While this approach is very effective in making explicit underlying confounding or biases, it may also be too rigid in that not every research question can be turned into a target trial ${ }^{19}$. Instead of disregarding these questions or the clinical care datasets that cannot answer them, it may be more fruitful to broaden the discussion on causal inference to include for example the Bradford Hill criteria ${ }^{18,19}$ or the concept of triangulation ${ }^{22}$. This may be especially relevant in the field of sex differences in dosing and side effects of medication, because true trial data are scarce and new trials are unlikely to be performed.

This section discussed how keeping in mind inherent limitations of clinical care datasets can help in both devising the best strategy to minimise their effects and weighing them against the strengths of those datasets. Striking the optimal balance between the two allows researchers to address clinically pressing evidence gaps, illustrated by the last three chapters of this thesis. However, these short-term strategies are not enough to make clinical care data-based research sustainable on the long term.

\section{The future perspective on clinical care data for research}

While it is important that proper methodology continues to be developed and taught in epidemiological and clinical curricula, methodological advances alone are not enough. Analytical tools can at best mitigate the effect of data quality issues, whereas improvements at the data-entry end can potentially prevent such issues from existing in the first place?. In addition, such improvements can radically reduce the time needed for data cleaning and organising ('data wrangling') that currently takes up $60-80 \%$ of time allocated for data science projects $^{23}$.

There are many different ways to improve data entry, including stimulating healthcare professionals to adhere to data entry protocols, creating a data collection infrastructure that supports healthcare professionals in reliable data entry, or building tools that automatically turn messy speech or free text data into structured variables, among others ${ }^{7,9,24}$. A combination of all these options will probably give the best results, but figuring out which combination is most viable requires the input from all parties involved in clinical care data ranging from healthcare professionals to scientists to ethicists to patients.

Reflecting on this thesis, a potential area for improvement would be structuring the collection of ADR-related information. Next to raising the quality of the collected data, it would also immensely reduce the data cleaning and structuring efforts needed to operationalise the recorded information. Plus, small adaptations can already make a world of difference during post-production data handling. Creating a dedicated data collection field 
for ADR information and making sure healthcare professionals record ADR information using that field only would already significantly narrow down the text fields researchers have to go through. Providing healthcare professionals with a drop-down menu containing the most commonly reported ADRs before opening up a free text field will make it easier to apply text mining algorithms because researchers do not have to account for all potential variations and errors in spelling. These and other lessons learned during clinical care data research can subsequently be incorporated in the development of an improved version of the data collection infrastructure, leading to better quality data in the long term. This way, several of the current inherent limitations of clinical care data will slowly be filtered out, further pushing the balance towards the potential of these datasets to shed light on currently understudied topics in sex differences research.

In summary, it seems that clinical care data have the potential to generate relevant and reliable data where there currently is none, given that a few inherent limitations are properly dealt with. Moreover, this potential will probably only grow in the future as a feedback loop between researchers and clinicians can drive improvements at the data entry end.The question remains, however, whether existing evidence gaps can be completely closed based on clinical care data alone.

\section{Closing existing evidence gaps, can clinical care data do it alone?}

When faced with a daunting lack of sex-specific data, clinical care datasets may currently be the only resource one can turn to as illustrated in this thesis. The works in this thesis generate momentum for sex differences research and push important sex-related questions into the spotlight. However, their influence somehow does not reach daily clinical practice and the patients that may benefit.

In the current evidence-based medicine landscape, observational data only has a small role to play compared with clinical trials, which are considered the pinnacle of reliable evidence ${ }^{25}$. This would mean that clinical care data can at most have a modest contribution in improving daily practice, even though they have so much potential. Clever study designs that incorporate the strengths of clinical care data into clinical trial settings, such as registry-based trials ${ }^{26}$, have been suggested and applied to expand the sphere of influence clinical care data can exert. This innovative approach to study design could also be valuable for sex differences research, especially because there are questions that cannot be answered with clinical care data alone. Chapter 6 and Chapter 7 both hint at benefits of sex-specific dosage but can never fully correct for the influences of confounding by indication and residual confounding. Narrowing down the optimal dosing practice for each sex requires proper randomisation and dosefinding trials. In these situations, clinical care data can contribute by informing the field about which specific medications might merit additional trials, directing the limited resources available to the most relevant questions. Additionally, they could serve as the selection pool 
from which trial participants are included in these trials. However, this will only work in combination with other improvements that combat the underrepresentation of women and lack of sex-stratified reporting. Otherwise, the percentage of women participating in clinical trials may remain too low for meaningful sex-specific analyses, illustrated by the recent LowDose Colchicine 2 trial where only I5\% of participants was female ${ }^{27}$.

Summarising, questions regarding sex-specific medication dosage need a combination of clinical care and clinical trial data to be answered. However, clinical care data-based evidence should be given more credit in other fields of sex differences research, especially regarding ADRs. Clinical trials may be able to distinguish true ADR risk from any nocebo effects thanks to randomisation ${ }^{28}$, but are otherwise not the best place to look for ADR data. With their highly specific study populations and relatively short follow-up times, trials are not powered to pick up on ADRs. Especially those that are rare or mainly occur in patient subgroups unlikely to meet the inclusion criteria. In addition, trials often actively exclude patients experiencing ADRs during the run-in period. Pharmacovigilance databases fill this gap, but lack information on prescription rates and patient-level characteristics. The latter in particular complicates the translation of pharmacovigilance findings to the clinic, because it is impossible to characterise the patient population the results pertain to. Clinical care data could have a big role to play here, especially when data entry becomes more standardised and of better quality.

To conclude, at the moment clinical care data offer many opportunities to generate interesting and relevant insights regarding existing evidence gaps, even though it cannot close them all without additional trial data. As the quality of clinical care data and its research is only expected to grow in the future, the potential will further outshine the pitfalls, making it a valuable source for sex differences research. 


\section{References}

I. Santema BT, Ouwerkerk W, Tromp J, et al. Identifying optimal doses of heart failure medications in men compared with women: a prospective, observational, cohort study. Lancet 2019; 394(I 0205): I 254-63.

2. Rosano GM, Lewis B, Agewall S, et al. Gender differences in the effect of cardiovascular drugs: a position document of the Working Group on Pharmacology and Drug Therapy of the ESC. Eur Heart J 20I5; 36(40): 2677-80.

3. Zopf Y, Rabe C, Neubert A, et al.Women encounter ADRs more often than do men. Eur J Clin Pharmacol 2008; 64(10): 999-1004.

4. Rademaker M. Do women have more adverse drug reactions? Am J Clin Dermatol 200I; 2(6): 349-5I.

5. Soldin OP, Mattison DR. Sex differences in pharmacokinetics and pharmacodynamics. Clin Pharmacokinet 2009; 48(3): I43-57.

6. Dunlay SM, Roger VL, Redfield MM. Epidemiology of heart failure with preserved ejection fraction. Nat Rev Cardiol 2017; I4(I0): 591-602.

7. Verheij RA, Curcin V, Delaney BC, McGilchrist MM. Possible Sources of Bias in Primary Care Electronic Health Record Data Use and Reuse.J Med Internet Res 2018; 20(5): el85.

8. Saracci R. Epidemiology in wonderland: Big Data and precision medicine. Eur J Epidemiol 20 I8; 33(3): $245-57$.

9. Weiner MG, Embi PJ. Toward reuse of clinical data for research and quality improvement: the end of the beginning? Ann Intern Med 2009; I 5 I (5): 359-60.

10. Hersh WR, Weiner MG, Embi PJ, et al. Caveats for the use of operational electronic health record data in comparative effectiveness research. Med Care 20I 3; 5 I (8 Suppl 3): S30-7.

II. Overhage JM, Overhage LM. Sensible use of observational clinical data. Stat Methods Med Res 20I3; 22(I): 7-13.

12. Chubak J, Pocobelli G, Weiss NS. Tradeoffs between accuracy measures for electronic health care data algorithms. J Clin Epidemiol 2012; 65(3): 343-9.e2.

13. von Lucadou M, Ganslandt T, Prokosch HU, Toddenroth D. Feasibility analysis of conducting observational studies with the electronic health record. BMC Med Inform Decis Mak 2019; 19(1): 202.

14. Weiskopf NG,Weng C. Methods and dimensions of electronic health record data quality assessment: enabling reuse for clinical research. J Am Med Inform Assoc 20I3; 20(I): I44-5I.

15. Ponikowski P,Voors AA, Anker SD, et al. 2016 ESC Guidelines for the Diagnosis and Treatment of Acute and Chronic Heart Failure. Rev Esp Cardiol (Engl Ed) 2016; 69(I2): II 67.

16. Shah SJ, Katz DH, Selvaraj S, et al. Phenomapping for novel classification of heart failure with preserved ejection fraction. Circulation 20I5; I3I(3): 269-79.

17. van Buuren S, Groothuis-Oudshoorn K. mice: Multivariate Imputation by Chained Equations in R. 20 I I 201 I; 45(3): 67.

18. Davey Smith G. Post-Modern Epidemiology:When Methods Meet Matter.Am J Epidemiol 2019; 188(8): I4I09.

19. Dekkers OM. The long and winding road to causality. Eur J Epidemiol 2019; 34(6): 533-5.

20. Elze MC, Gregson J, Baber U, et al. Comparison of Propensity Score Methods and Covariate Adjustment: Evaluation in 4 Cardiovascular Studies. J Am Coll Cardiol 2017; 69(3): 345-57.

21. Hernán MA, JM R. Causal Inferece:What If.: Boca Raton: Chapman \& Hall/CRC; 2020.

22. Lawlor DA, Tilling K, Davey Smith G. Triangulation in aetiological epidemiology. Int J Epidemiol 20I6; 45(6): I866-86.

23. Press G. Cleaning Big Data: Most Time-Consuming, Least Enjoyable Data Science Task, Survey Says. 2016. https://www.forbes.com/sites/gilpress/2016/03/23/data-preparation-most-time-consuming-least-enjoyabledata-science-task-survey-says/ (accessed II March 202I).

24. Meystre SM, Lovis C, Bürkle T, Tognola G, Budrionis A, Lehmann CU. Clinical Data Reuse or Secondary Use: Current Status and Potential Future Progress. Yearb Med Inform 20I 7; 26(I): 38-52.

25. Murad MH,Asi N,Alsawas M,Alahdab F. New evidence pyramid. Evidence Based Medicine 20I6; 2 I (4): I25-7. 


\section{CHAPTER 8}

26. James S, Rao SV, Granger CB. Registry-based randomized clinical trials--a new clinical trial paradigm. Nat Rev Cardiol 20I5; I2(5): 312-6.

27. Nidorf SM, Fiolet ATL, Mosterd A, et al. Colchicine in Patients with Chronic Coronary Disease. New England Journal of Medicine 2020; 383(19): I838-47.

28. Gupta A, Thompson D, Whitehouse A, et al. Adverse events associated with unblinded, but not with blinded, statin therapy in the Anglo-Scandinavian Cardiac Outcomes Trial-Lipid-Lowering Arm (ASCOT-LLA): a randomised double-blind placebo-controlled trial and its non-randomised non-blind extension phase. The Lancet 20I7; 389(I0088): 2473-8I. 
GENERAL DISCUSSION 

Appendix 


\section{Nederlandse samenvatting}

Vrouwen zijn anders dan mannen. Dat lijkt misschien een open deur, maar in wetenschappelijk onderzoek naar hart-en vaatziekten worden vrouwen en mannen nog te vaak over één kam geschoren. Vrouwen worden minder vaak gevraagd deel te nemen aan wetenschappelijk onderzoek, waardoor de onderzoeksresultaten voornamelijk gebaseerd zijn op mannen. Daarnaast worden deze resultaten zelden voor vrouwen en mannen apart gepresenteerd, zelfs als er wel voldoende vrouwen meedoen aan het onderzoek. Hierdoor is er een gebrek aan geslachtsspecifieke kennis, onder andere over de optimale behandeling van vrouwen en mannen met hartfalen. Hartfalen is een hart- en vaatziekte waarbij het hart onvoldoende bloed door het lichaam pompt. Het ziekteproces dat tot hartfalen leidt, is anders bij vrouwen dan bij mannen. Er zijn ook verschillen in hoe een vrouwenlichaam medicatie metaboliseert in vergelijking met een mannenlichaam, waardoor vrouwen anders kunnen reageren op behandeling. Het is daarom opvallend dat vrouwen zijn ondervertegenwoordigd in klinische trials die kijken naar de werkzaamheid en veiligheid van hartfalen medicatie. In dit proefschrift hebben we onderzocht of reguliere zorgdata dit gebrek aan kennis kunnen opheffen. Reguliere zorgdata bestaan uit elektronische patiëntendossiers en vormen een dwarsdoorsnede van de patiëntenpopulatie die dagelijks in de zorg gezien wordt. Hierdoor zijn vrouwen wel evenredig vertegenwoordigd in deze data.Wij denken daarom dat reguliere zorgdata een belangrijke bijdrage kunnen leveren aan het dichten van kennishiaten over man-vrouw verschillen in de werkzaamheid en veiligheid van medicijnen.

Het eerste deel van dit proefschrift benadrukt het schrijnende tekort aan geslachtsspecifieke informatie over bijwerkingen van medicatie voor hartfalen. In hoofdstuk $\mathbf{2}$ laten we zien dat maar twee van de 23 gepubliceerde klinische trials over deze medicijnen (9\%) geslachtsspecifieke informatie over bijwerkingen verstrekken. Als we ook ander onderzoek naast klinische trials meenemen in de zoektocht door de wetenschappelijke literatuur, wordt dit percentage zelfs nog lager: 7\% (II van de 155 studies). Dit tonen we aan in hoofdstuk 3. Deze twee hoofdstukken laten zien dat de huidige aanpak niet werkt en dat we vanuit een nieuwe invalshoek moeten zoeken naar mogelijkheden om man-vrouw verschillen in het risico op bijwerkingen te onderzoeken.

Wij denken dat reguliere zorgdata deze nieuwe invalshoek kunnen zijn en introduceren in hoofdstuk 4 de reguliere zorgdatabase van Cardiologie Centra Nederland (CCN). Deze database omvat een kleine II0.000 patiënten die door hun huisarts op verdenking van een hartaandoening naar één van de $13 \mathrm{CCN}$ locaties zijn verwezen voor een uitgebreide check-up. Tijdens deze check-up wordt ook gedetailleerde informatie verzameld over het medicijngebruik van de patiënt, waaronder bijvoorbeeld de dosering en mogelijke bijwerkingen. Daarnaast is ongeveer de helf van alle patiënten vrouw (52\%), waardoor deze dataset erg geschikt is voor de vraagstelling van dit proefschrift. We gebruiken deze dataset in de hierop volgende drie hoofdstukken. 
In hoofdstuk 5 laten we zien dat vrouwen vaker vanwege een bijwerking met hun cardiovasculaire medicatie stoppen dan mannen, zowel in absolute aantallen als na correctie voor het totaal aantal recepten per geslacht. Dit verschilt per geneesmiddel en varieert van II\% tot $50 \%$ meer bijwerkingen bij vrouwen na correctie voor recepten. Vrouwen melden voor dezelfde geneesmiddelen ook andere bijwerkingen dan mannen. Zo hebben vrouwen vaker last van hoofdpijn, duizeligheid, allergische reacties en buikpijn, terwijl mannen vaker hart- of nier-gerelateerde klachten hebben. Dit hoofdstuk benadrukt dat het risico op bijwerkingen verschilt tussen vrouwen en mannen. Vanuit veiligheidsperspectief vraagt dit dan ook om een geslachtsspecifieke(re) benadering van medicamenteuze behandeling.

In hoofdstuk 6 benaderen we man-vrouw verschillen in medicatie vanuit het werkzaamheidsperspectief. We laten zien dat de optimale dosering van medicatie voor hartfalen mogelijk lager is voor vrouwen dan voor mannen. In tegenstelling tot mannen, hebben vrouwen een lager overlijdensrisico wanneer ze de helft of minder van de door de richtlijn aangeraden dosis voorgeschreven krijgen. Deze bevinding neemt de huidige unisex behandelingsrichtlijnen op de schop en stelt de vraag of een geslachtsspecifieke aanpak geen betere zorg oplevert voor zowel vrouwen als mannen met hartfalen.

In hoofdstuk 7 kijken we naar man-vrouw verschillen in de werkzaamheid van statines, cholesterolverlagend middelen. Hoewel de werkzaamheid van deze middelen meerdere malen bewezen is, worden ze minder vaak en in lagere doseringen aan vrouwen voorgeschreven. We laten zien dat statines de kans op overlijden verlagen bij zowel vrouwen als mannen en dat dit effect zelfs sterker is bij vrouwen. Een hogere dosering heeft een sterker effect dan een lagere dosering, maar het verschil is marginaal. Dit hoofdstuk neemt resterende twijfels over de effectiviteit van statines bij vrouwen weg en suggereert dat zelfs een lage dosering al een groot effect kan hebben.

Deze laatste drie hoofdstukken etaleren de mogelijkheden van klinische zorgdata om kennishiaten over man-vrouw verschillen op te pakken en op te lossen. Het blijft echter de vraag in hoeverre een kennishiaat daadwerkelijk kan worden gedicht op basis van alleen klinische zorgdata. Ook deze data hebben namelijk beperkingen. In hoofdstuk 8 bespreken we hoe de inherente beperkingen van klinische zorgdata de bevindingen van de eerdere hoofdstukken beïnloeden, verkennen we strategieën om met deze beperkingen om te gaan, en beantwoorden we de vraag of klinische zorgdata voldoende in staat zijn om bestaande kennishiaten omtrent man-vrouw verschillen in effectiviteit en veiligheid van geneesmiddelen op te vullen. 


\section{Dankwoord}

"Het is pas uit als ik zeg dat het uit is.

.... Het is uit."

En daarmee zijn we bij de laatste pagina's van dit proefschrift aanbeland. Een proefschrift dat er zonder alle mensen om mij heen niet had kunnen liggen. Bij deze wil ik iedereen bedanken die direct danwel indirect heeft bijgedragen aan mijn promotietraject. In mijn eentje was ik nooit zo ver gekomen.

Prof. dr. ir. den Ruijter, beste Hester. Het voelt goed om die 'prof' neer te mogen zetten en jou in de functie van promotor te hebben staan. Je bent een inspirerend voorbeeld van hoe je op eigen termen en via je eigen weg je doelen kunt bereiken of onderwerpen die je aan het hart liggen (pun intended) op de kaart kunt zetten. Dat je daarvoor niet altijd via de gebaande wegen hoeft te gaan of alle puntjes hoeft aan te tikken, was voor een lijstjesmens zoals ik soms lastig te accepteren. Met je vrijere aanpak trok je me meerdere keren uit mijn comfortzone, en hoewel dat niet altijd even soepel verliep, heb ik daardoor wel veel geleerd zowel op inhoudelijk als persoonlijk vlak. Ik heb bewondering voor je nooit aflatende stroom aan nieuwe ideeën en enthousiasme voor de wetenschap, en de manier waarop je altijd precies de essentie van een project in een paar zinnen kan verwoorden. Bedankt dat je me de ruimte gaf om mijn eigen plekje te vinden toen bleek dat ik liever opschoof richting de epidemiologie. En wees gerust, welke kleur mijn haar ook heeft, mijn eerste vraag over welk onderzoek dan ook zal altijd zijn:“Goed verhaal, maar hoe zit dat bij vrouwen?”.

Dr. Onland-Moret, beste Charlotte. Ik ben ontzettend blij dat jij in het tweede jaar van mijn promotietraject aanhaakte en sindsien de stabiele (epidemiologie) factor in mijn projecten bent geweest. Je hielp me de structuur hervinden als ik weer eens verloren was in de chaos van een project (okee, maar wat is ook alweer de onderzoeksvraag?) of verstrikt was geraakt in mijn planning (okee, maar wat heeft hier nou prioriteit?). Je maakte de epidemiologie die ik zo gemist had weer één van de centrale pijlers van mijn promotietraject. Ik kon altijd goed met je sparren over methodologische vraagstukken, nieuwe ideeën voor een epi-project, of de domme (sorry, ik weet dat ik dat eigenlijk niet mag zeggen) opmerkingen van de reviewers. Ik vind het heel leuk dat ik in jou een gelijkgestemde heb gevonden om dit soort onderwerpen mee te bespreken, bedankt daarvoor.

Prof. dr. Pasterkamp, beste Gerard. Ondanks dat ik niet bij jou geëindigd ben, wil ik je graag bedanken voor je begeleiding als promotor gedurende het eerste deel van mijn promotie. Tijdens onze maandelijkse gesprekken wist je me altijd een nieuw perspectief te geven om mee naar mijn werk te kijken. Het 'Write your Future' programma en de bijbehorende reis naar Amerika is één van de hoogepunten van mijn promotietijd.Van het vroegtijdig durven stoppen met projecten waar weinig in lijkt te zitten (kill your darlings) tot de vraag waarom je diabetes type 2 zou willen voorkómen (treating it is cheaper), de lessen van die reis staan me nog steeds bij. 
Dr. Vaartjes en prof. dr. Asselbergs, beste Ilonca en Folkert. Bedankt voor jullie inzet als mijn begeleidingscommissie. Toen ik het even niet meer zag zitten, boden de gesprekken met jullie en de tips die daaruit voorkwamen mij de handvaten om weer uit de put te klimmen waar ik in zat.

Graag wil ik de leden van de leescommissie, te weten prof. dr. Boersma, prof. dr. Hooft, prof. dr. Pasterkamp, prof. dr. ir.van der Schouw en prof. dr. Sturkenboom, bedanken voor het lezen en beoordelen van mijn proefschrift.

Ook wil ik alle co-auteurs bedanken voor de mooie publicaties en prettige samenwerking. Specifiek wil ik dr. Somsen en dr. Tulevski van Cardiologie Centra Nederland bedanken voor hun inzichten vanuit de praktijk.

Dan alle mede-promovendi en andere collega's waar ik de afgelopen vier jaar mee gewerkt heb. De 'Write your Future' club, bedankt voor de gezelligheid, de meetings (en borrels) in De Branding om aan ons projectvoorstel te werken, en de ervaringen in Amerika. Dear members of the Asselbergs brainstorming meeting group, thank you for your feedback on my projects and the interesting insights I gained from listening to you presenting your own work each week. Leden van de Geoffrey Rose, bedankt voor jullie kritische vragen en kijkjes in de keuken.All people of the Experimental Cardiology group, whether it was chasing sheep in real life or solving Christmas riddles online, thank you for all the fun. Robin, ik kon altijd bij je terecht om even lekker te zeiken onder genot van een kopje koffie. Bedankt voor alle geweldige gifjes, vines, het meezingen met foute hitjes tijdens borrels, en het OSSD avontuur. Heel veel succes met de volgende stap in je carrière, hopelijk kom je snel een keer door de moorderende concurrentie heen daar in Amerika. Elise, het was altijd een plezier om met jou samen te werken, of het nou een paper was, een brainstorm dag, of een grant voorstel over een onderwerp waar we eigenlijk niks van af wisten dat last-minute nog even moest worden ingediend. Ik wens je het beste! Daniek, Naomi en Mark, bedankt voor de weekstart, brainstormdagen, journal clubs, en gezelligheid tijdens de jaarlijkse barbecue en de borrels. Michele and Ernest, I haven't seen much of you due to me not being lab-based and of course the corona issues, but I had fun chatting with you both on- and offline and I hope you have a good time in Hester's group.

Ik wil ook de studenten bedanken die ik (deels) heb mogen begeleiden, en twee daarvan in het bijzonder. Thuur, volgens mij hebben we je niet altijd de leukste kanten van wetenschap laten zien maar met je humor sloeg je je overal gewoon doorheen. Zure snoepjes zijn het beste, daar blijf ik bij. Milena, ik voel me vereerd dat je twee keer stage bij me wilde lopen omdat je het zo leuk vond. Onze meetings gingen bijna vaker niet over de inhoud dan wel, maar dat maakte werken met jou juist zo'n plezier. Wat jullie hierna ook gaan doen, het wordt sowieso een succes.

Alle Torenbewoners past and present, bedankt voor de afgelopen vier jaar aan koffiemomentjes, paas- en kerstbrunches, en gedeeld leed over electriciteit die om de dag uitvalt en de passieve 
agressie van de mensen van de overkant. Michael, jouw R-skills hebben me waarschijnlijk dagen aan geklooi gescheeld en de kattenplaatjes in je Outlook uitnodigingen waren on point. Ik moet zeggen dat ik een beetje jaloers was op je werkplekje met airco bij Skyline het afgelopen jaar, maar het is je gegund. Malin, ons kamergenootschap was kort, maar het was leuk om te zien hoe je je draai vond binnen de wondere wereld van de wetenschap. Gideon, de rook van verbrande kaiserbroodjes zal je in dit gezelschap blijven achtervolgen vrees ik. Je was de sociale noot, altijd in voor een borrel of uitje. Ik heb veel lol gehad tijdens het samen door New York struinen en Tim uit elke Apple Store trekken die we tegenkwamen. Enja, je was mijn reddingsboei toen ik aan het verdrinken was in de CCN dataset. Saskia, bedankt voor je warme welkom in de Toren met mijn eerste echte kantoorkoffiemok en alle ondersteuning vanuit de data-management. Sander, altijd leuk als je even bij ons kwam buurten met een gekke vraag of interessante tip, en ik kon de Sultans of Swing op de vrijdagmiddag ook erg waarderen. Aisha en Anouk, jullie zetten me als kersverse promovendus stevig op de grond en hielpen me aarden in de Toren en in de CCN dataset. Ian, van vrolijke goedemorgen liedjes tot een shotjes dispenser die op je bureau past, bedankt voor alle leuke momenten. Anne-Mar, van samen in de Toren naar R-service aan huis, ondanks de afstand heb ik de laatste jaren fijn met je samengewerkt. De saga rondom je moestuin was een paar weken het hoogtepunt van onze wekelijkse online meetings, leuk dat ik hem ook in het echt heb kunnen zien. Diantha, volgens mij hebben we elkaar vaker online gezien en gesproken dan in het echt, maar ik vond het gezellig om samen 'op de kamer' te zitten. Ik vind het bewonderingswaardig hoe snel je alles oppakt en regelt zo vanuit huis. Ik wens jullie allemaal het beste en hoop jullie nog eens tegen te komen, in professionele setting of gewoon voor een drankje.

Tim, nadat je mij op mijn eerste dag vakkundig had rondgeleid door het UMCU en de Toren had ik je in mijn hoofd al op een voetstuk gezet als 'die collega die tegelijkertijd met je begint maar alles al weet'. Dat voetstuk werd in onze eerste paar maanden hardhandig afgebroken door onze computers die maar niet kwamen, de wijsheden van InspiroBot, en de goede gesprekken die we voerden over werkelijk alles. Bedankt voor alle zin en onzin die je de afgelopen vier jaar met me gedeeld hebt. Of het nou de Uithof is, New York (ah yes, your kind lives there), Japan (subarashiiii) of je nieuwe huis in Amsterdam, met jou is het altijd lachen.

Allerliefste \#0900-Troostpot-Hotline. Of het nou huilen van het lachen of huilen met de pet op was, volgens mij hebben alle mogelijke emoties bij ons de revue gepasseerd de afgelopen paar jaren. En wat een jaren waren het. Floor, emotionele steunpilaar en stille kracht. Ik zie je nog voor de deur staan met een fles champagne een paar uur voor mijn proefschrift deadline en kan me de repen Tony's die ineens op mijn bureau lagen de ochtend na een zware dag ook nog goed herinneren. Hoogtepunt of dieptepunt (sorry, leermoment), je was er altijd om het samen te vieren of samen een halfuurtje stoom af te blazen bij GenMab. Ik weet niet of mijn schrijfskills alle lof verdienen die jij ze toedicht, maar ik schrijf met liefde nog eens wat met (voor?) je. Je toekomstige collega's en patienten mogen zich in hun handen 
knijpen met je. Jonne, spil van het torenleven en redder van menig promovendus. Als ik de weg kwijt was in alle bureacratie, wist jij wie ik moest mailen of welke folder ik moest hebben. Als ik in een project ergens niet uitkwam, wist jij hoe ik mijn (soms te sterke) mening moest omvormen naar effectief professioneel taalgebruik. En als ik iets leuks wilde doen, had je het al geregeld, of het nou New York of fietsen naar Parijs of een weekendje Disneyland was. Het is ontzettend leuk om je weer met plezier te zien werken bij de Hartstichting en te zien genieten van Eli. Klaske, eeuwige optimist en doorzetter. Toen je net begon was ik onder de indruk van je enthousiasme en werklust (en sociale agenda), en dat ben ik eigenlijk nog steeds. Bijna elke klaagsessie bij jou eindigde met een opbeurende 'en door', uit die negativiteit en op naar de volgende stap. Bedankt dat je CCN samen met me gedragen hebt, en elke donderdag met me spierpijn hebt geleden nadat we op de bootcamp woensdag weer allemaal rare dingen hadden gedaan. $\mathrm{lk}$ kan niet wachten totdat ik je zelfontworpen kleurenpalet voor je boekje in levende lijve mag aanschouwen. Meidenmeiden, ik had me geen betere collega's kunnen wensen. Snel weer een keer een G\&T doen?

Dearest members of the, well, I don't know how we currently call ourselves, but you know who you are Henry, Esther, Mike, Cami, Johannes and Franco. Thank you for all the shenanigans and banter. Whether it is climbing, boardgames, videogames, or just chilling and vibing, hanging out with you is always fun and sure to take my mind of any work-related troubles I might have. On the downside I now no longer know what constitutes a sandwich, but that's a small price to pay. Let's go fall our way up some rocks again soon.

UCU Leedees, wij daten waaay back natuurlijk. Myrt, het enige verschil tussen in Hong Kong zitten of in Zeist is dat we nu naast elkaar zittend commentaar kunnen leveren op k-drama's in plaats van over de app. Bedankt voor het verbreden van mijn horizon en je absolute planloosheid waardoor we eigenlijk altijd wel kunnen afspreken als we daar zin in hebben. Mir, bedankt voor vier jaar aan 'Happy Monday' berichtjes, sporadische avonturen in Oxford, bijkletsen via videocall, en verhalen over zoete aardappelen en Papoea New Guinea. Ik heb absoluut noken wari over het afronden van jouw DPhil en kom je graag bewonderen in je mooie afstudeer pakje volgend jaar. Tiets, met je drukke agenda konden we elkaar niet altijd even vaak zien, maar als je er was dan klikte het weer zoals vanouds en konden we lachen over de kleinste dingen. Ben heel benieuwd naar je nieuwe huisje in Groningen als het eindelijk presenteerbaar bevonden wordt, ik weet al welke theepot ik als housewarming gift zal meenemen. Taar, we hebben altijd de interessantste gesprekken over de gekste onderwerpen. Of het nou over heel serieuze onderwerpen gaat of niet, jij hebt altijd wel een opmerking of invalshoek die ik zelf niet zou bedenken. Laten we snel weer eens een wandelingetje doen, maar dan bij voorkeur niet in de vrieskou.

Lieve familie, de Eijdems clan en de Botsen, bedankt voor de familie-events, verjaardagen, en alles wat er zich in de familie apps afspeelt. Leuk om de 'neefjes en nichtjes' van vroeger te zien opgroeien, en dat we nu bij elkaar op housewarming kunnen of leden van de 'oude garde' 
bij ons thuis kunnen uitnodigen. Mark, Maud en Max, bedankt voor de gezellige etentjes. Alle oud-bewoners van de $\mathbf{K 9 0}$ en partners, bedankt voor de kerstdiners, nieuwjaarsuitjes, en Viking Kubb sessies. Marco en Marjon, bedankt voor de steun en de goede gesprekken over wat ik nou wil met mijn toekomst en hoe dan en waar.

Koen, mijn jongen, ben je er klaar voor? Dat maakt niet uit, we wachten toch niet op je. When suddenly ik van gewoon nerd gepromoveerd ben naar hooggeleerde tata. En ja, I am pleased about it. Alhoewel, technisch zeergeleerd, stiekem is Beertje de enige echt hooggeleerde tata, maar dat laten we nu even. Bedankt voor je humor, alle filmpjes en stickers en andere onzin die je met me deelt zodat ik nog een beetje meekom met de 'echte studenten' en cool kan doen tegenover mijn eigen vrienden. Maar ook voor de fietsrondjes, je nieuwe passie voor planten, de bordspelletjesavonden met Nanette, en de iets serieuzere gesprekken die we dan voeren. Heel veel succes met afronden van je master, en dan buizen amice?

Lieve mama, bedankt voor alles. Lieve papa, jij mocht er ook zijn. Nee maar zonder grapjes, bedankt dat jullie altijd voor me klaar staan met een kop koffie, een knuffel, en een goed advies. Papa, ik kon altijd lekker hardop met je nadenken over de inhoud als ik weer eens vastzat of dacht dat ik een goed idee had. Mama, ik kon al mijn sociale en 'soft skill' struggles met je bespreken, ontleden waar het probleem lag, en bedenken wat ik als vervolgstap het beste kon doen. Door jullie onvoorwaardelijke steun kan ik zijn wie ik ben, trots gaan staan waar ik nu sta, en vol goede moed de toekomst in stappen. Ik hou van jullie.

Kasper, je ziet de bui al hangen of niet? Als laatste krijg je natuurlijk het meest zoetsappige verhaal. Nee, ik zal het je besparen. Je weet zelf het beste hoe onmisbaar je bent in mijn leven, hoe ik zonder je nooit alle heuvels, muren, en bergen op mijn pad had kunnen beklimmen. Quite literally, want zonder jou was ik nooit aan boulderen begonnen. Bedankt voor je geduld als ik weer eens futloos op de bank lag en alleen maar pizza wilde bestellen, bedankt voor al je advies en het aan me trekken als ik de volgende stap niet durfde te zetten, bedankt voor alle leuke momenten en uitjes en game avonden en vakanties en cocktails. Met jou kan ik de wereld aan. Ik hou van je. 


\section{List of publications}

\section{Published}

Bots SH, Siegersma KR, Onland-Moret NC, Asselbergs FW, Somsen GA, Tulevski II, den Ruijter HM, Hofstra L. Routine clinical care data from thirteen cardiac outpatient clinics - design of the Cardiology Centers of the Netherlands (CCN) database. BMC Cardiovasc Disord. 202I; 2 I (I):287.

Groepenhoff F, Klaassen RGM, Valstar GB, Bots SH, Onland-Moret NC, Den Ruijter HM, et al. Evaluation of non-invasive imaging parameters in coronary microvascular disease: a systematic review. BMC Med Imaging. 202I;2I (I):5.

Bots SH, Inia JA, Peters SAE. Medication Adherence After Acute Coronary Syndrome in Women Compared With Men:A Systematic Review and Meta-Analysis. Frontiers in Global Women's Health. 2021;2(I).

Groepenhoff F, Eikendal ALM, Onland-Moret NC, Bots SH, Menken R, Tulevski, II, et al. Coronary artery disease prediction in women and men using chest pain characteristics and risk factors: an observational study in outpatient clinics. BMJ Open. 2020;10(4):e035928.

Groepenhoff F, Eikendal ALM, Bots SH, van Ommen AM, Overmars LM, Kapteijn D, et al. Cardiovascular imaging of women and men visiting the outpatient clinic with chest pain or discomfort: design and rationale of the ARGUS Study. BMJ Open. 2020; I0(I2):e0407 I 2.

Bots SH, Groepenhoff F, Kessler EL, Sickinghe AA, Eikendal ALM, Leiner T, et al. Sex-Specific Aspects in the Pathophysiology and Imaging of Coronary Macro- and Microvascular Disease. J Cardiovasc Transl Res. 2020; I3(I):39-46.

Valstar GB, Bots SH, Groepenhoff F, Gohar A, Rutten FH, Leiner T, et al. Discovery of biomarkers for the presence and progression of left ventricular diastolic dysfunction and HEart failure with Preserved ejection Fraction in patients at risk for cardiovascular disease: rationale and design of the HELPFul case-cohort study in a Dutch cardiology outpatient clinic. BMJ Open. 2019;9(6):e028408.

Bots SH, Groepenhoff F, Eikendal ALM, Tannenbaum C, Rochon PA, Regitz-Zagrosek V, et al. Adverse Drug Reactions to Guideline-Recommended Heart Failure Drugs in Women: A Systematic Review of the Literature. JACC Heart Fail. 2019;7(3):258-66.

Bots SH, den Ruijter HM. Recommended Heart Failure Medications and Adverse Drug Reactions in Women. Circulation. 20 I 9; I39(I2): I469-7I.

Peters SAE, Bots SH, Woodward M. Sex Differences in the Association Between Measures of General and Central Adiposity and the Risk of Myocardial Infarction: Results From the UK Biobank. J Am Heart Assoc. 20 18;7(5).

Bots SH, Zuidgeest MGP, Gohar A, Eikendal ALM, Petrelli A, van Os-Medendorp H, et al. Women in Translational Medicine:Tools to Break the Glass Ceiling. Front Med (Lausanne). 20I8;5:330. 
Bots SH, Peters SAE, Woodward M. Sex differences in coronary heart disease and stroke mortality: a global assessment of the effect of ageing between 1980 and 2010. BMJ Glob Health. 2017;2(2):e000298.

Backholer K, Peters SAE, Bots SH, Peeters A, Huxley RR, Woodward M. Sex differences in the relationship between socioeconomic status and cardiovascular disease: a systematic review and meta-analysis. J Epidemiol Community Health. 20I 7;7I (6):550-7.

Bots SH, van der Graaf Y, Nathoe HM, de Borst G], Kappelle JL, Visseren FL, et al. The influence of baseline risk on the relation between $\mathrm{HbAlc}$ and risk for new cardiovascular events and mortality in patients with type 2 diabetes and symptomatic cardiovascular disease. Cardiovasc Diabetol. 2016;15(I): I0I.

\section{Submitted}

Bots SH, Onland-Moret NC, Tulevski II, van der Harst P, Cramer MJM, Asselbergs FW, Somsen GA, den Ruijter HM. Heart failure medication dosage and survival in women and men seen at outpatient clinics.

Bots SH, Onland-Moret NC, Jancev M, Hollander M,Tulevski II, Hofstra L, Somsen GA, den Ruijter HM. Statins reduce mortality risk to a greater extent in women compared with men in a cardiac outpatient population.

\section{In preparation}

Bots SH, Jancev M, Onland-Moret NC, Tulevski II, Somsen GA, den Ruijter HM. Sex differences in the number and type of adverse drug reactions reported for common cardiovascular medications in a cardiac outpatient population.

Bots SH, Groenwold RHH, Dekkers OM. Using electronic health record data for research: a quick guide.

Bots SH, Schreuder MM, Watson S, van Puijenbroek E, Onland-Moret NC, den Ruijter HM, Roeters van Lennep JE. Sex differences in adverse drug reactions to angiotensin-converting enzyme inhibitors. 


\section{Curriculum Vitae}

Sophie Bots was born on 26 February 1994 in Rotterdam, the Netherlands. She started her academic career at University College Utrecht with a BSc in premedical science. Her bachelor thesis sparked her interest for epidemiological research, which she developed further during a research internship at the George Institute for Global Health. The MSc in Global Health Science at the University of Oxford was a logical next step to learn all required skills and tools for epidemiological research and get a

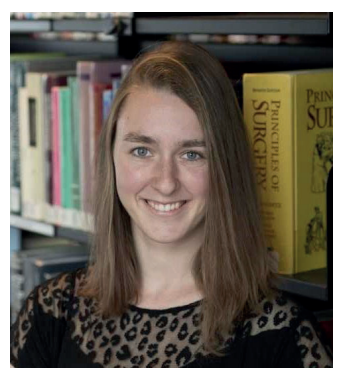
taste of where these tools could be applied.After finishing her masters in 2017, she started her $\mathrm{PhD}$ on sex differences in cardiovascular disease at the University Medical Center Utrecht under supervision of prof. dr. ir. Hester den Ruijter and dr. Charlotte Onland-Moret. The results of her work as a PhD Candidate are described in this thesis.

During her $\mathrm{PhD}$, she turned raw clinical data from roughly I 10,000 patients into a relational database that forms the basis of both her own work and several research projects from other PhD candidates and MSc students. Through her experiences with clinical care data, she encountered many challenges that come with using such data for research. She realised that researchers often do not have sufficient tools or knowledge to properly deal with these challenges, a problem she hopes to address in her future work. 


